



\title{
Root morphology of co-occurring \\ African fruit tree species \\ with contrasting strategies of exploration and exploitation
}

\author{
Dissertation \\ zur Erlangung des Doktorgrades \\ an der Fakultät für Forstwissenschaften und Waldökologie \\ der Georg-August-Universität Göttingen
}

vorgelegt von

geboren in

Göttingen
Armin Oppelt

Bamberg

2003 


\section{Bibliografische Information Der Deutschen Bibliothek}

Die Deutsche Bibliothek verzeichnet diese Publikation in der Deutschen Nationalbibliografie; detaillierte bibliografische Daten sind im Internet über http://dnb.ddb.de abrufbar.

1. Aufl. - Göttingen : Cuvillier, 2004

Zugl.: Göttingen, Univ., Diss., 2003

ISBN 3-89873-985-6

D 7

1. Berichterstatter Prof. Dr. Douglas L. Godbold

2. Berichterstatter Prof. Dr. Winfried Kurth

3. Berichterstatterin Prof. Dr. Andrea Polle

Tag der mündlichen Prüfung $\quad 09.05 .2003$

http://webdoc.sub.gwdg.de/diss/2004/oppelt/index.htm

(C) CUVILLIER VERLAG, Göttingen 2004

Nonnenstieg 8, 37075 Göttingen

Telefon: 0551-54724-0

Telefax: 0551-54724-21

www.cuvillier.de

Alle Rechte vorbehalten. Ohne ausdrückliche Genehmigung des Verlages ist es nicht gestattet, das Buch oder Teile daraus auf fotomechanischem Weg (Fotokopie, Mikrokopie) zu vervielfältigen.

1. Auflage, 2004

Gedruckt auf säurefreiem Papier

ISBN 3-89873-985-6 
Table of Contents

List of abbreviations

1 Summary

2 General Introduction

3 Monographs of tree species

4 Results and Discussion

4.1 Methods

4.2 Site description and climate

4.3 Morphological distinctions

4.4 Fractal Aspects

4.4.1 Coarse roots

4.4.2 Fine roots

4.5 Topological and metrical aspects

4.6 Exploration and Exploitation

4.6.1 Morphology, Exploration and Exploitation by fine roots

4.6.2 Spatial distribution, Exploration and Exploitation by coarse roots

5 Supplementary comparisons

5.1 Fractal dimension and Topology

5.2 Fractal dimension and Exploration 22

5.2.1 Coarse roots 22

5.2.2 Fine roots

5.3 Topology and Exploration

5.4 Exploration and other parameters

\subsubsection{Coarse roots}

5.4.2 Fine roots 


\section{Publications}

I Structure and Fractal dimension of root systems of four co-occurring fruit tree species from Botswana

II Topology, scaling relations and Leonardo's rule in root systems of African tree species

III Contrasting rooting patterns of some arid-zone fruit tree species from Botswana - I. Fine root distribution

IV Contrasting rooting patterns of some arid-zone fruit tree species from Botswana - II. Coarse root distribution Curriculum Vitae 


\section{List of abbreviations}

a altitude (topological parameter)

b mean toplogical depth (topological parameter)

D spatial box counting dimension (3-D, coarse roots)

Dwt fine root dry weight

$D_{x y} \quad$ planar box counting dimension (2-D, coarse roots and fine roots)

$E(0) \quad$ exploration index (coarse roots and fine roots)

$E(\phi) \quad$ exploitation index (coarse roots and fine roots)

EE exploration efficiency (coarse roots)

$\operatorname{GEE}(\phi)$ generalized efficiency of exploitation (coarse roots)

ISV individual soil volume (coarse roots)

$k \quad$ exponent of fitting equation for exploitation index $E(\phi)$ (fine roots)

$l_{e} \quad$ mean exterior link length (coarse roots)

$l_{i} \quad$ mean interior link length (coarse roots)

$n(0) \quad$ number of soil cores containing roots (fine roots) or number of "cubes" containing any root (coarse roots)

$n(\phi) \quad$ number of cores samples with $S A R>\phi$ (fine roots) or number of "cubes" containing roots with $R V D>\phi$ (coarse roots)

NEC number of explored cells (coarse roots)

$P R L \quad$ relative percentage of total root length (coarse roots)

$q_{a} \quad$ normed index - corresponding to altitude a

$q_{b} \quad$ normed index - corresponding to mean topological depth $b$

$R A D \quad$ root surface area density (fine roots)

$R L D \quad$ root length density (coarse roots)

RVD root volume density (coarse roots)

SAR root surface area (fine roots)

$v \quad$ total number of links (coarse roots)

$v_{0} \quad$ magnitude - number of exterior links (coarse roots) 



\section{Summary}

Measurement of in situ grown and completely excavated coarse root systems (five individuals per species) from Grewia flava DC (Tiliaceae), Strychnos cocculoides BAK, Strychnos spinosa LAM (Loganiaceae) and Vangueria infausta BURCH (Rubiaceae) were used to derive several parameters describing explorative and exploitative features of the selected species. Additionally, systematic soil sampling was carried out to achieve information about morphological patterns of fine roots and data about spatial fine root distribution. Comparisons between fine root and coarse root characteristics are considered and correlations, concerning the spatial distribution of both entities, are calculated. Main focus was addressed to speciesdependent architectural differences in order to select 'suitable', i.e. less competitive rooting patterns, sought-after for integration into sustainable agroforestry systems.

Each coarse root system was reconstructed with means of semi-automatic digitising in order to obtain 3-D models and to calculate the spatial distribution of root length density $(R L D)$ and root volume density $(R V D)$, respectively. With the aid of 3-D reconstruction it was possible to extract a large amount of information serving for various quantitative analysis. Apart from morphological and architectural distinctions, fractal characteristics, topological parameters, supplemented by link lengths analysis, dependencies between diameter and branching, as well as scaling relations were investigated, founded on a precise data basis. Furthermore, the spatial information was useful to quantify and assess several aspects of "exploration" and "exploitation", also including efficiency considerations, in order to estimate the potential competition of the selected species with agroforestry crops.

Measurements of fractal dimension $D$ were conducted for comparison of space filling patterns and also to detect species-dependent differences. Discretisation of reconstructed spatial patterns, aided by the software GROGRA, made it possible to determine fractal box counting dimension $D$ of complete, three-dimensional coarse root systems, thus bringing an improvement compared to recent literature where fractality was mostly investigated in small, herbaceous plants and using only 2Dprojections. However, the box counting dimension $D_{x y}$ of two-dimensional projections was also calculated, in order to compare both measurements with each other. Although the box counting dimension - for $D$ and $D_{x y}$ - yields only very condensed information about spatial organization of the branching structure, results confirm different rooting strategies already obtained by visual inspection in the field and are also in accordance with other parameters obtained.

Going beyond the commonly used topological parameters, two new indices, $q_{a}$ and $q_{b}$, related to the well-known graph-theoretical altitude a and mean topological depth 
$b$, were developed. The introduction of these normed indices, confined to the interval [0;1], takes the different sizes of coarse root systems into account and ensures independence from planarity assumptions. Topological analysis was additionally complemented with analysis of external and internal link lengths. As topology also influences the source acquisition, the idea was born to compare architectural and topological features with the ability of exploration and exploitation. However, conscious that the used notions are normally not explicitly defined in the literature and also often used ambiguously, several quantitative methods, already established and applied in several investigations, were tested on the sample trees. An attempt was undertaken to clarify both notions and to apply already established parameters to the investigated coarse root systems and also to fine root samples.

The exploration index $E(0)$ and the exploitation index $E(\phi)$ were introduced and applied to coarse root systems as well as to fine root samples. In order to analyse potential competition of the coarse roots, the following parameters were additionally introduced: In an attempt to approximate soil volume explored by coarse roots, the parameter number of explored cells NEC was used. With the exploration efficiency $E E$ and the new parameter generalized efficiency of exploitation $G E E(\phi)$, efficiency aspects of exploration and exploitation, respectively, are also quantified. Thus, definitions from the literature, describing explorative and exploitative aspects, were further extended and generalized, in order to clarify commonly used notions.

Analysis of fine roots was carried out with digital image-analysis, using subsamples to measure the morphological parameters length, surface area, volume and average diameter of fine root samples, as well as the planar box counting dimension $D_{x y}$. Correlations between morphological parameters and dry weight (Dwt) were used to approximate whole fine root distribution and to evaluate species-specific differences in terms of competition.

The variation of ages and therefore different spatial extension of each single root system were taken into account by introducing an individual soil volume ISV adapted to each individual coarse root system. This volume was determined by the horizontal spread of the coarse roots and the coring depth of the fine root samples $(80 \mathrm{~cm})$. This accurate determination was beneficial, because a differing fine root distribution was detected inside and outside the ISV, and the considered rooting depth proved to adequately represent whole coarse root systems.

Clear distinctions arising from the analysis of the box counting dimension $D$ have shown that coarse root systems from Strychnos cocculoides, with distinctively lowest values, were most contrasting with those from Vangueria infausta. In this regard Grewia flava and Strychnos spinosa have to be considered as intermediate species. Apparent evidence that $D$ values were well correlated with $R L D$ are in agreement 
with the interpretation that the box counting dimension measures the tendency to fill space.

Findings from the topological assay, expressed as $q_{a}$ and $q_{b}$ values, resulted in evident differences, indicating that coarse root systems from Strychnos cocculoides were most of all approaching a "herringbone" branching pattern whereas Vangueria infausta, and Grewia flava, did more approximate a "dichotomous" configuration. Strychnos spinosa stands between both extremes. Results about mean exterior link lengths $\left(I_{e}\right)$ are in close agreement with the above mentioned distinctions on branching patterns: samples from Strychnos cocculoides, representing the most herringbone-like type, and even S. spinosa, representing more intermediate patterns, were characterized by distinctively higher mean values, in contrast to the more dichotomous coarse root samples. Hence, the distinctive branching patterns are also correlated with the metric parameter $l_{e}$. In contrast, no species-specific differences were found for mean interior link lengths $l_{i}$. The empirical test of the theoretical assumption of Leonardo's rule, not yet tested on other coarse root systems, confirmed the "pipe stem theory" with reasonable accuracy. Furthermore, the relation between root collar diameter and total coarse root length respectively volume, often discussed but seldom applied to empirical data, yielded good correlations, independent of the considered species. However, analysis of a tapering rule, based on the theory of elastic similarity, did not show convincing results.

Comparing the values from the exploration index $E(0)$ for a rooting depth between 0 and $80 \mathrm{~cm}$, fine and coarse root distribution from Strychnos cocculoides turned out to be the least explorative species as opposed to the most competitive Vangueria infausta. Looking only at the fine roots, $E(0)$ values for both Strychnos species, were similarly low, indicating low competition levels, whereas a clearly transitional stage was assigned to Grewia flava samples. Vangueria infausta, as the most competitive representative, could be separated from all other species, and Strychnos cocculoides was still significantly different from the intermediate Grewia flava, For the coarse roots, variable and significant species-dependent differences were observed for $E(0)$, dependent on varying rooting depth. Considering a soil depth between 0 and $80 \mathrm{~cm}$, Strychnos spinosa and Grewia flava were clearly standing between the most contrasting species Strychnos cocculoides and Vangueria infausta. Dependent on rooting depth, differences between the investigated species were even more pronounced for the upper soil layers. These differences lessened with increasing rooting depth. This can be explained by already decreasing values for $E(0)$ of coarse roots for Grewia flava and Vangueria infausta samples for a depth below $40 \mathrm{~cm}$, whereas values of exploration indices were continuously increasing for both Strychnos species with increasing soil depths. In terms of NEC, Grewia flava and Vangueria infausta, with distinctively highest values, 'compete with each other' for a 
superior position. But significant species-specific differences for NEC were only achieved when age was considered as a covariate. Looking at the efficiency of exploration $(E E)$, compared with $E(0)$ respectively $N E C$, it can be assumed that highly explorative species gain their outstanding status only with high efficiency, whereas those with low explorative ability do not reach this level of efficiency.

Regarding exploitation by fine roots, clear species-dependent differences (expressed as $k$ values) occurred: Vangueria infausta, with highest value of exploitation, could be significantly distinguished from all other investigated species. Less distinct results were attained for the exploitation index for the coarse roots. $E(\phi)$ did not induce a clear ranking between the species. In contrast, for the parameter $G E E(\phi)$, also only applied to coarse roots, estimating the efficiency of exploitation, showed clear distinctions between the species, independent of considered $\phi$ values. Strychnos cocculoides was assigned the lowest mean value as opposed to Grewia flava. Ranking between the species was similar to that from $E E$, indicating that efficiency of exploration and efficiency of exploitation are closely related with each other.

In general, the results presented here do not support the frequently discussed hypothesis that a clear trade-off between exploration and exploitation exists. In the majority of cases, Strychnos cocculoides was always most contrasting with Vangueria infausta. Depending on the considered parameters Grewia flava and Strychnos spinosa were assigned intermediate patterns with more or less affinity to one of both extremes. These trends were well associated with topological distinctions, indicating a more herringbone configuration for both Strychnos species. Hence, features that clearly favour low competitive attitudes (deep rooting with pronounced tap root, associated with weakly branched laterals and even weak development of fine roots). In most instances, either Grewia flava or Vangueria infausta were most contrasting to Strychnos cocculoides. Comparing these results with the output from topological analysis, the observations coincide with a dichotomous branching configuration, associated with an intensive and dense network of roots, especially in topsoil layers. Hence, among the investigated species Strychnos cocculoides can be clearly favoured for the integration into simultaneous agroforestry systems. Whereas shallow rooting architecture, additionally associated with high explorative and exploitative potential and also with an intensive fine root development, contributes to a high potential competition with agroforestry crops. Samples from Strychnos spinosa stand between both extremes, but here, too, dependent on the considered parameters, the species can express a more or less competitive potential. Some tentative conclusions, concerning the general construction plans of coarse root systems, can be drawn: topological distinctions seem to be obviously associated with different morphological and/or architectural patterns, features that clearly affect explorative and exploitative behaviour. This also 
determines the extend of species-dependent potential belowground competition. Whether this is a general construction plan also inherent to other species, can be elucidated only with further investigation. Comparison between coarse and fine root characteristics confirmed a tendency towards a 'common strategy'. For example, intensity of fine root development, easily obtained in the field, seem to be suitable to predict certain architectural features of their coarse root systems (and vice versa). These patterns can be used to estimate explorative and/or competitive ability of the species. The existence of contrasting branching patterns of co-occurring species can be interpreted as an effective means of spatial and/or temporal sharing of soilderived sources. These distinctive features might be exceptionally important in arid and nutrient-poor habitats. Although it cannot be concluded which branching pattern is definitely more advantageous, the information gained supports decisions for the selection of 'suitable' species with low competition for simultaneous agroforestry systems.

The present work demonstrates that complete excavation combined with systematic core sampling can be a suitable method to obtain detailed information about the composition of whole root systems and to distinguish species-related differences. With the help of this approach it was possible to confirm the theory of contrasting rooting patterns in the same habitat. With the data obtained, species could be classified according to their potential competition with agroforestry crops. 


\section{General Introduction}

The main focus of the present work was to distinguish rooting patterns of in situ grown species, Strychnos cocculoides BAK, S. spinosa LAM (Loganiaceae), Grewia flava DC (Tiliaceae) and Vangueria infausta BURCH (Rubiaceae). These indigenous fruit trees are an important food source, especially for people practising subsistence dryland farming, because they yield crops even when arable agriculture fails, improving food security for rural households. Therefore, their suitability for integration into simultaneous agroforestry systems is of vital interest. In order to ensure sustainable yield benefits, a combination between trees and crops is necessary where spatial and temporal sharing between soil-derived resources is possible (Cannell et al. 1996). But for a possible co-existence with annual crops their rooting patterns must be 'suitable' in terms of competition. For integration of potential perennials into agricultural systems it is recommended that competition between trees and crops can be reduced by selecting species with limited lateral root extension and/or deep rooting species. Therefore, species-dependent rooting patterns were analysed, in order to evaluate the suitability of the investigated species for simultaneous agroforestry systems.

But, the key question appeared: May co-occurring species develop only similar or even contrasting rooting patterns when grown in the same habitat? Proceeding from the assumption that distinctive patterns of rooting architecture might occur, different parameters were tested, aiming at quantitative methods, mainly discussed under the aspect of competition.

Several agroforestry research efforts, however with contrasting results, have not yet managed to conclude which factors, either above or belowground, are more important for a successful co-existence of perennials and annual crops (e.g. Singh et al. 1989, Ong et al. 1991, Rao et al. 1991, De Costa and Chandrapala 2000a, $2000 \mathrm{~b}$ ). But results tend to indicate that belowground competition increases with severe drought conditions (Ong et al. 1991).

Main attention is turned to the investigation of whole root systems, including information on fine root distribution as well as architectural aspects of entire coarse root systems. But thought has also been given to aspects of complementarity considering potential soil conservation. 


\section{Monographs of tree species}

\section{Grewia flava DC.}

Tiliaceae (Brandy Bush, Velvet Raisin, Wild raisin, Wild currant).

Mostly a common shrub with numerous thin branchlets up to $2 \mathrm{~m}$ tall that can even develop into a small tree up to $4 \mathrm{~m}$ height. It is widely distributed and native to dry deciduous woodland and bushveld in the southern African region, in particular in the Kalahari (Palgrave 1977). This species appears mainly on sandy soils and is found from Zambia to Kwazulu-Natal. In undisturbed areas, it commonly appears beneath Acacia erioloba trees, indicating their propagation through frugivorous birds (Schurr 2001). But, also indigenous browsers as well as domestic life stock are considered as potential seed propagators, presumably accounting for a more homogeneous encroachment of Grewia flava into tree interspaces (Mphinyane 2001). The influence of domestic livestock, which promotes an evenly distributed spatial population through accelerated and uniform dispersion of seeds, is currently discussed. Both types of seed dispersal seem to be an important determinant of Grewia encroachment. Grewia flava, as a heavily browsed species, especially during the dry season, is known to coppice profusely.

The smooth bark is dark grey or grey-brown to black. Bark fibres may be used to make ropes and to weave baskets. Branches are used as walking sticks and San people used to make their bows and arrows from this species. Small branches with frayed ends can be even used as toothbrushes (Palgrave 1977). Pegs from twigs, driven into the ground, are used as protection against lightning.

The upright held leaves are greyish-green with fine hairs on the upper side and a more dense hair cover at the paler green bottom side. The elliptic or oblanceolate leaves are quite small (length: $1.4-7 \mathrm{~cm}$, width: $0.7-2.5 \mathrm{~cm}$ ).

The yellow, star-shaped flowers (approximately $1.5 \mathrm{~cm}$ in diameter) appear in short branched axilliary heads between October to March (Palgrave 1977).

The almost spherical fruits, approximately $10 \mathrm{~mm}$ in diameter, ripen from February onwards, and can be gathered until August, turn into red- or orange-brown when ripe. They are eaten in large quantities, because they have more flesh than other Grewia species, or even sold in some (Matsheng) villages (Taylor and Moss 1982). The sweet and also slightly astringent fruits, with only a thin layer of flesh, are directly eaten or dried and then ground or mashed, soaked in water, to produce a porridge. In certain areas dried fruits are used to brew a beer (Kgadi-traditional beer) or distil a type of brandy (Palmer and Pitman 1972, Giess 1985). Stamped fruits, mixed with dry locusts, are greatly esteemed as delicacy by Tswana people. Dried and crushed 
seeds are used in porridge. Additionally, Terfeza pfeilii, a potato-like fungus that lives in symbiosis with Grewia flava, has to be considered as a serious by-product (Palgrave 1977).

\section{Strychnos cocculoides BAK.}

Loganiaceae (Corky-bark Monkey Orange, Monkey Orange, Bush Orange, Wild Orange)

An occasional tree up to 5 (8) $\mathrm{m}$ in height with a compact rounded crown, growing on both deep and loamy sands. This, fairly fast growing, species occurs in woodlands, mixed forests, deciduous woodlands, lowlands, and Miombo woodlands (FAO 1983). Preferred sites are deep sandy soils as well as rocky hill slopes (Palmer and Pitman 1972), but this species is also growing on black to dark-grey clays and yellow-red loamy sands (FAO 1983). Its distribution comprises large parts of Africa (also equatorial regions) and is also native to southern Africa (Wehmeyer 1966, Fox and Norwood-Young 1982, Taylor 1986). Due to its value as fruit-bearing species, $S$. cocculoides is retained and protected, even when fields are cleared. Therefore, it can be considered as a semicultivated species. It prefers open growing conditions, indicating high light demands. Therefore, weeds and climbers need to be removed until trees are established. In a study from VPR \& D (Mateke 2001) it was shown, that shade can have a positive effect on the development of young seedlings, however, a prolonged shelter increases attacks of powdery mildew. As seeds do not germinate readily, pretreatment of soaking seeds in hot water $(24-48 \mathrm{~h})$ is recommended for a successful germination (Regional Soil Conservation Unit 1992), but sowing season might also cause different germination rates. Seedlings can be raised in a nursery and planted on a cleared site. However, rapid transplanting from seedling bags is essential for an undisturbed development of the root structure. In the wild, annual fires soften the seed coat and accelerate germination. However, saplings need to be protected from fire. Also vegetative propagation, through coppicing and root suckers, by wounding the tree (fire, trampling animals), is reported to be easy.

The broadly ovate-oblong to almost circular and leathery leaves are opposite and conspicuously 5 -veined from the base. Leaf size ranges from approximately 2.5 to 5 $\mathrm{cm}$ length and 1.5 to $4 \mathrm{~cm}$ width. The upper surface is sometimes roughly hairy but mostly without hairs and then shiny, while the bottom side is paler green and dull.

The small flowers, about $5 \mathrm{~mm}$ in diameter, are green to creamy white, appear during the rainy season as aggregated terminal dense clusters (up to $3.5 \mathrm{~cm}$ ) on short lateral branchlets.

The hard, circular, woody-shelled and distinct white speckled fruits are smooth, about 7 to $10 \mathrm{~cm}$ in diameter, turn from dark green to yellowish or orange when ripe. 
However, fruits from superior phenotypes might even reach a diameter of $15 \mathrm{~cm}$ (Taylor 1986). Ripe fruits have a pleasant taste. The sweet fruit flesh is sucked from the kernel and is popular, especially amongst younger people because it is sweeter than the flesh of $S$. pungens. If eaten in large quantities though, it can cause stomach pain and diarrhoea. The fruits are also used for distilling a strong spirit. The specific name, indicating 'like a small grain' refer to the smaller fruits of the type specimen. The large bony seeds, which are said to be poisonous (Palmer and Pitman 1972, v. Koenen 1996), are covered by a white edible fruit flesh. Fruits ripen during the dry season (April to August); this process can take up to a year. Within the genus, $S$. cocculoides is considered to be the best in terms of eating quality (Palgrave 1977). Ripe fruits emit a delicate aroma reminiscent of the spice clove. Fruits are sought after, and therefore, in Namibia green fruits are harvested and buried in sand pits (called ete), in order to ripen in the warm sand but also to reduce competition with other fellow human beings for that fruits. Ungrafted trees are reported to produce first fruits within 4 to 5 years. Yield in the wild is estimated with 300 to 400 fruits per tree.

This species can be easily recognized through its distinctive corky, lengthwise and deeply ridged bark, which is creamy-brown. Main branches are quite thick and armed with strong curved spines. Smaller branches are often ending in a terminal spine.

A dye, prepared from fruits, serves to colour trays and containers, to provide protection from insect attacks. The middle part of the bark is used to cure stomach pain. It is cooked in water and the decoction is drunk. Fresh leaves are used for healing wounds: pounded, mixed with water and heated, a tincture is applied that dries out wounds and protects them from getting infected. Drinking a mixture of unripe, mashed fruits, which are soaked for a certain time in water, induces vomiting within a short time, probably caused by substances of the green shell. Ripe pulp, mixed with honey or sugar is used to treat coughing. The root can be chewed to alleviate eczema and is an alleged cure for gonorrhoea (FAO 1983).

The wood is white and tough, rather soft, and pliable. It is used primarily for building materials and tool handles (Palgrave 1977).

\section{Strychnos spinosa LAM.}

Loganiaceae (Elephant Orange, Monkey Ball, Natal Orange, Spiny Monkey Ball, Spiny Monkey Orange, Kaffir Orange)

A semi-deciduous tree up to $7 \mathrm{~m}$ in height. In Madagascar this species is considered as an indicator plant of degraded grassland.

The bark is ground and taken as a remedy for poisoning people inflicted by witchcraft. 
Fruits from S. spinosa are distinctively bigger than those of $S$. cocculoides. They are as large as a quince, with a gourd-like shell full of large flat seeds imbedded in the fleshy pulp. Beside $S$. cocculoides, fruits from this species are most popularly consumed. Both are considered as the better tasting ones within that genus (Palgrave 1977). Unripe fruit can be to some people poisonous. As a significant amount of trade in wild fruits at urban markets exists, S. spinosa has also to be mentioned as another promising species with great potential for domestication. Similar to the above described S. cocculoides, this species is also widely distributed throughout Africa. Removing seeds from fruit flesh and soaking them in water is recommended for a successful germination.

Regarding the fruits, the confusion between the two species, $S$. cocculoides and $S$. spinosa, may well be deliberate: $S$. spinosa is generally claimed to have the more delicious fruit, but this is a fallacy cunningly encouraged by the local people. In fact $S$. cocculoides has unquestionably the more delectable flavour and hope, in this manner, the deliberate confusion is made to safeguard this species and enjoy its fruit themselves (Palgrave 1977).

Several parts are traditionally used. Roots are used in production of eardrops and also used as a remedy for fevers and inflamed eyes. A decoction of fruits is used to cure stomachache and treat bronchitis. Pulp from ripe fruits, is either eaten fresh or dried and stored for later use. It is somewhat acid and said to be delicious, because it also contains citric acid. Shells remaining after pulp has been extracted are used for crafts and musical instruments. The inner skin of fruit shells contains valuable oils that have potential in cosmetics. Whereas unripe fruits and also seeds are used to induce vomiting to treat snakebite victims. Strychnine and strychnine alkaloids, which have been identified, are believed to be the active ingredients for treatment against snakebite. Leaf infusions provide lotion for sore eyes and also other analgesic remedies are made from decoction of leaves.

\section{Vangueria infausta subsp. infausta BURCH.}

Rubiaceae (Wild medlar, False medlar).

This usually multi-stemmed large bush, or less often, a small deciduous tree, reaches a height of approximately 2 to 6 (12) $\mathrm{m}$ depending on soils and moisture. It grows on all kinds of deep sand, preferably in thick shrubby areas very often in the shade of larger species. Due to its fruits, this species is popular throughout the savannah woodlands of Eastern and Southern Africa (e.g., Fox and Norwood-Young, 1982). The dark green and felt leaves are opposite, soft and densely covered with hairs. The small flowers (diameter about $6 \mathrm{~mm}$ ) form greenish yellow clusters, which appear from October to January. The fruits are almost circular, up to $4 \mathrm{~cm}$ in diameter, 
turning from green to brown-orange when ripe (Palgrave 1977). Ungraftet trees are reported to bear fruits within the first 8 months. The raw, soft, slightly mealy pulp tastes similar to wild apple, but also reminds to fig fruits. However, it is sweet and refreshing and is well liked. They have a thin skin and contain several (1-3) longish seeds, which are covered by an orange flesh (Palmer and Pitman 1972). Fruit size varies in a wide range $(2.5$ to $5 \mathrm{~cm})$. As an average, an adult tree produces approximately 1500 fruits. Observations on field trials have shown good performance, because two year old trees with a height of $1.3 \mathrm{~m}$ bore already as much as 400 fruits. This result may be very encouraging for domestication of $V$. infausta (Taylor et al. 1986). However, drought or erratic rainfall causes fruits to abort. Fruits ripen from January onwards and can be gathered up to May. A sporadic germination is reported, which can be reduced by scarifying the seeds or with treatment of hydrogen peroxide (Msanga and Maghembe 1989). Here too, the rate of germination depends on the season when seeds are sown.

When the fruits start getting dry from April onwards, they are soaked in water for a certain time, then boiled and mashed to prepare a kind of porridge. Fresh fruits cannot be stored for more than a week, but sun dried they can be stored for almost one year. The fruit is an important food source with high potential for commercial use. Due to its species name 'infausta', except from the fruits, other products are not used for domestic purposes, because of spiritual beliefs.

Among the investigated species, Vangueria infausta has shown highest preference to VAM, however, with variations of infection, depending on site and seasonal conditions (Bohrer 2001, Bohrer et al. 2001). These results suggest, that success of this species highly depends on Interactions with VAM fungi. 


\section{Results and Discussion}

\subsection{Methods}

In order to quantify whole root system composition, knowledge about coarse root architecture as well as information from the fine root distribution and their morphology are required. Aiming at species-specific differences of root architecture of four cooccurring tree species, coarse root systems from five individuals per species were manually excavated. Additionally, before excavation of each single root system, systematic core sampling was carried out. For more detailed description of the methods see Publication I and II.

Coarse and fine root spatial characteristics were used to identify and quantify similarities as well as differences in the rooting behaviour of co-occurring species. Coordinates from each systematic core sample can be correlated with any arbitrary coordinate from the corresponding coarse root system. This spatial information was used to calculate nearest neighbour relations between fine root distribution and the position of coarse roots. As core samples were either located within or outside the soil volume enclosed by coarse roots, the determination of an individual soil volume (ISV) was defined to determine different patterns of fine root distribution (cf. Publication III and IV). Hereby, the combination of reconstructing whole coarse root systems with the additional integration of fine root sampling data presents a new technique in order to obtain more detailed information about whole root system architecture. With the possibility of comparing different entities, common as well as contrasting patterns between and within the species could be determined. Furthermore, several theoretical assumptions are applied to coarse root systems (cf. Publication I and II).

\subsection{Site description and climate}

The investigation site was located in Central District of Botswana between longitude $26^{\circ} 36.26^{\prime}-36.70^{\prime} \mathrm{E}$ and latitude $22^{\circ} 25.09^{\prime}-25.30^{\prime} \mathrm{S}$. Sample trees were naturally grown in an untilled habitat. Deep aeolic sands, with an arid moisture regime (Soil Survey Staff 1999), form the soils, Typic Torripsamments (LCBG). A low fertility status, especially in organic and iron content, is characteristic for that region.

The climate can be described as arid with mean annual precipitation reaching approximately $650 \mathrm{~mm}$ in the extreme northeast, with a clear decrease towards southwest (about $250 \mathrm{~mm}$ ). The country, prone to drought, receives the majority of rainfalls in the summer months, between October and April, generally as scattered, high intensity, short-duration thunder showers. The mean annual rainfall for the Serowe region reached approximately $400 \mathrm{~mm}$. 
Temperature variations are extreme throughout the year and also vary greatly within the daily cycle. In winter, daytime temperatures above $20^{\circ} \mathrm{C}$ are reached, but in the early hours of the morning they can drop to about $0{ }^{\circ} \mathrm{C}$. Temperatures during the summer vary from $12-15{ }^{\circ} \mathrm{C}$ during the early morning to $30-40{ }^{\circ} \mathrm{C}$ in the late afternoon.

The vegetation of the investigation site, a tree savannah, belongs to the Terminalia sericea, Lonchocarpus nelsii / Acacia erioloba association. This 'Northern Kalahari Tree and Bush Savannah' is a widespread vegetation zone on sandveld.

\subsection{Morphological distinctions}

Obvious morphological differences were found for both, coarse and fine root patterns. In terms of coarse root architecture, Grewia flava and Vangueria infausta have to be regarded as shallow rooting species with a dense network of structural roots. In contrast Strychnos cocculoides turned out to be a deep rooting species, with only a weak development of lateral coarse roots whereas Strychnos spinosa, with a more intensive development of structural roots, shows an intermediate type (cf. Publication I and II).

Distinctive morphological features were found for the fine roots. Furthermore, the proportion of fine root containing cores $E(0)$ was highest for Vangueria infausta and lowest for Strychnos cocculoides samples, whereas values for $E(0)$ from Strychnos spinosa was only marginally higher than those from the latter mentioned species. In terms of fine root containing cores, Grewia flava turned out to be an intermediate type. Additionally, in terms of fine root surface area, Vangueria infausta signficantly exceeded mean values from all other investigated species. More detailed information can be found in Publication III and IV.

\subsection{Fractal Aspects}

Since Mandelbrot's (1977) seminal work, the complexity of forms and patterns in nature is often described in terms of fractal geometry. With the introduction of noninteger dimensions, this method provides a more integrated measure of plant architecture and growth than can be provided by traditional Euclidean geometry. (cf. Publication I for theory of fractals).

\subsubsection{Coarse roots}

The fractal characteristic was analysed by determining the three-dimesional box counting dimension $D$ on the complete coarse root systems. Strychnos cocculoides, with apparently the weakest coarse root system development, contrasted most with Vangueria infausta. Grewia flava and Strychnos spinosa did not reveal significant 
differences neither from each other nor from all remaining species. Hence, their root systems have to be considered as intermediate. (cf. Publication I).

As most studies only apply planar projections of the fractals (Berntson 1996), results were also compared with the planar box counting dimension $D_{x y}$. Both dimensions were positively correlated with each other and $D_{x y}$ was not systematically diminished from three dimesional assumptions. Hence, it can be assumed that the twodimensional model does not misrepresent this aspect in the three-dimensional situation. However, more investigations are necessary to confirm these assumptions. Nevertheless, significant differences in $D$ point at obviously contrasting rooting patterns in the same habitat.

As the fractal analysis is well-known as a useful tool to quantify different patterns of space occupation, results were further compared with different degrees of exploration and exploitation (cf. Chapter 5).

\subsubsection{Fine roots}

Mean values for the box counting dimension of fine roots for the planar projections showed no significant inter-species differences. However, an inverse trend was observed, when mean values of $D_{x y}$ from fine roots for the single species were compared with either $D$ or $D_{x y}$ from the whole coarse root systems. High mean values for $D_{x y}$ of fine root samples were correlated with low values for $D$ respectively $D_{x y}$ of the coarse root systems and vice versa, probably indicating a multifractal behaviour (Mark and Aronson 1984, Kaye 1989, Mandelbrot 1989).

\subsection{Topological and metrical aspects}

Topological as well as metrical parameters of the coarse root systems were also analysed. Additionally to the already well established topological distinctions herringbone and dichotomous two new indices $\left(q_{a}, q_{b}\right)$ were introduced. The analysis was further complemented by examination of link lengths analysis, diameter and scaling relations. (cf. Publication II).

In addition, some well-known hypotheses about geometry and scaling relations (e.g. pipe stem theory, tapering rules), with main emphasis on the relationship between proximal root diameter and several size-dependent parameters were analysed. As those theories are mostly used in theoretical models, however, often without empirical basis, confirmation or rejection of these assumptions were placed in the foreground.

Strychnos cocculoides with the highest mean values for $q_{a}$ and $q_{b}$, indicating a nearly herringbone structure, whereas Grewia flava and Vangueria infausta, with distinctively lower values for $q_{a}$ and $q_{b}$, approach more to dichotomous patterns. 
Strychnos spinosa was shown to be an intermediate type. Differences between the species were even more pronounced for this analysis than for the classical topological parameters (Fitter 1987), using altitude a or mean topological depth $b$.

Results from link lengths analysis yielded species related differences for external link lengths $\left(I_{e}\right)$, dividing the considered species into two subgroups: Strychnos cocculoides and S. spinosa vs. Grewia flava and Vangueria infausta. However, internal link lengths $\left(l_{i}\right)$ did not reveal species-dependent differences.

The pipe stem theory, dating back to Leonardo da Vinci, could be confirmed for the investigated coarse root systems with reasonable accuracy (cf. Publication II).

The relationship between proximal root diameter vs. root length and root volume, respectively, corroborated several assumptions of various models (e.g., van Noordwijk 1994, Spek and van Noordwijk 1994). However, the path length analysis (McMahon and Kronauer 1976), originally derived from mechanical self-similarity assumptions in tree crowns, did not yield convincing results (Publication II).

\subsection{Exploration and Exploitation}

In order to quantify the method, estimating exploration and exploitation, based on the definition of Hughes et al. (1995) was applied. Where possible, parameters were used in an analogous manner, applying them for both entities, coarse and fine roots. The definition of the terms exploration and exploitation is however not a trivial issue, because these expressions are often used in an ambiguous sense with different context or meaning. In analogy to Hughes et al. (1995), the following parameters are analysed: The "exploration index" $E(0)$, defined as the proportion that contains any root in relation to the potential soil volume where roots may occur. Whereas the "exploitation index" $E(\phi)$ quantifies the fraction of soil volume that is enclosed by roots, exceeding a specified, arbitrary threshold value $\phi$. Value for $\phi$ can be any parameter, either the density of root length $(R L D)$, root surface area $(R A D)$ or root volume $(R V D)$.

Both indices, primarily adopted to fine roots, were tested on fine root samples and further extended and applied to coarse root systems. Furthermore, the parameters "exploitation potential" (Berntson 1994) and "exploitation efficiency" (Fitter 1987, Fitter et al. 1991) were only tested for the considered coarse root systems. However, as defined by Hughes et al. (1995), the exploitation potential, defined as the sum of each root surrounding soil volume, and also the exploitation efficiency, expressed as relation between sum of depletion zone volume and total root system volume, quantify exploration rather than exploitation. Therefore, some of the parameters were renamed according to the notions of exploration and exploitation used by Hughes et al. (1995): instead of exploitation potential the term NEC (number of explored cells), 
estimated as cubic cells surrounding each single coarse root, was coined. The notion exploitation efficiency is subsequently termed as exploration efficiency (EE). (cf. Publication IV, Table 1). The main intention was that new definitions enabling rigorous distinctions between the terms as well as the analysis enables further elucidation of the frequently used but so far not clearly defined terms "exploration" and "exploitation". As a consequence of this systematisation, a new index determining the efficiency of exploitation - generalized exploitation efficiency $(\operatorname{GEE}(\phi))$ - was also introduced and adopted for the coarse root systems. The parameter $\operatorname{GEE}(\phi)$ was defined as the ratio between the amount of grid cells $n(\phi)$ containing roots with $R V D>\phi$ and total root volume, it clearly characterizes exploitative patterns. (cf. Publication III and IV).

\subsubsection{Morphology, Exploration and Exploitation by fine roots}

Instead of commonly used root length densities $(R L D)$, root surface area densities $(R A D)$ were used to describe morphological features of the fine roots. Morphological analysis was carried out using digital image analysis. Furthermore, speciesdependent differences in the spatial distribution were investigated. Due to morphological distinctions and also in order to describe physiologically important parameters RAD appeared as a more adequate parameter than RLD (cf. Publication III).

In terms of both $R A D$ and $E(0)$, highest mean values were always reached for Vangueria infausta, which was most contrasting with fine roots samples from Strychnos cocculoides in both parameters. Mean values for $E(0)$ from Grewia flava samples were between both extremes and those from Strychnos spinosa were close to that of $S$. cocculoides, pointing at a close genus-relation. However for RAD, Grewia flava had the lowest mean values, due to numerous, but very thin capillary fine roots (Publication III).

Therefore in terms of fine roots, expressed either as RAD or $E(0)$ Vangueria infausta has to be considered as most competitive whereas Strychnos cocculoides, with low values for both parameters, and additionally a spatial concentration of coarse roots, that favours the accumulation of fine roots in deeper soil layers were clearly most contrasting (cf. Publication IV).

Exploitation by fine roots, expressed as $E(\phi)$, has shown that the least explorative species Strychnos cocculoides had also lowest exploitative ability, whereas the most explorative species Vangueria infausta was also most exploitative. Similar to the results on exploration, Grewia flava and Strychnos spinosa represent intermediate types (cf. Publication III). 


\subsubsection{Spatial distribution, Exploration and Exploitation by coarse roots}

For exploration and exploitation of coarse root systems the parameters root length density $(R L D)$ and root volume density $(R V D)$ were used. As the architecture of structural roots determines the spatial extension of the entire root system, root length has to be considered as the primary factor. Furthermore, RVD was also considered, as the root volume is mainly responsible for the re-generation of new roots and is therefore responsible for the potential competition of root systems. Especially in terms of exploitation, this parameter seemed to reveal more reliable results, it was therefore incorporated in the parameters determining exploration efficiency $(E E)$ and generalized efficiency of exploitation (GEE $(\phi))$ (cf. Publication IV).

For the cumulative percentage of root length $(P R L)$ and only considering the top soil layers $(0-20 \mathrm{~cm})$, two contrasting groups - Grewia flava and Vangueria infausta, with distinctively higher values vs. both Strychnos species - could be distinguished. But with increasing rooting depth, only Strychnos cocculoides could be separated from the other species. Also the horizontal distribution revealed similar results (Publication IV).

As a consequence of the above described distribution of $P R L$, similar contrasting, species-dependent differences were also found for the exploration index $E(0)$. Regarding different rooting depths, distinctions were more pronounced for upper soil layers, but until a rooting depth between 0 and $80 \mathrm{~cm}$, Vangueria infausta turned out to be the most and Strychnos cocculoides the least explorative species. Strychnos spinosa and Grewia flava, with almost similar values for $E(0)$, were located between both extremes (Publication IV). This result suggests a 'common strategy' between fine and coarse roots for the single species.

In contrast to the commonly discussed trade-offs between exploration and exploitation (cf. Fitter 1985, Berntson 1994), the correlation between exploration efficiency $(E E)$ and number of explored cells $(N E C)$ was clearly positive. However, the different scales - coarse root systems vs. small herbaceous plants might be the cause of contrasting results. In terms of exploration efficiency, Grewia flava has to be considered as more efficient than the more explorative Vangueria infausta (expressed as $E(0)$ ), although both yielded similar values for the exploration index $E(0)$. When NEC was analysed, age had to be included as a covariate in order to detect species-dependent differences (Publication IV). Possible advantages and disadvantages of deep vs. shallow rooting patterns (cf. Schwinning and Ehleringer 2001) and consequences of the distinction between herringbone and dichotomous rooting patterns (cf. Fitter, 1987, Fitter et al., 1991) are further discussed in Publication IV. 
The parameter $E(\phi)$, suitable for the distinction of the species in terms of fine roots, did not yield significant results when applied to coarse root systems. However, the new index $G E E(\phi)$, introduced in order to quantify the efficiency of exploitation, revealed clear species-dependent differences. Analogous to the results of exploration efficiency $(E E)$, similar ranking was achieved for the parameter $G E E(\phi)$, with maximum values for Grewia flava and minimum values for Strychnos cocculoides. Therefore, it can be concluded that species efficient in exploration are also efficient in exploitation (Publication IV).

Patterns for the relationship between the vicinity of coarse and fine roots were obviously different, depending on the location of soil cores (inside or outside the ISV). Therefore, the individual soil volume (ISV) turned out to be very useful in order to detect species-related and species-independent differences in the spatial distribution of fine roots. Results generally coincide with investigations from Gersani et al. (2001), discussing interplant and intraplant interactions, and also with conclusion from Tardieu et al. (1992), investigating the tendency of root clumping.

All parameters with their abbreviations, used to analyse different rooting patterns, and the significant species-related distinctions imposed by them are summarized in Table 1.

Further comparisons between the results presented in Chapter $4.3-4.6$ were made in order to accept or reject hypotheses about common and/or contrasting features between and within the species (cf. Chapter 5). 
Table 1. Recapitulating overview of different analysed parameters from fruit tree species Grewia flava (G.f.), Strychnos cocculoides (S.c.), Strychnos spinosa (S.s.) and Vangueria infausta (V.i.) with investigations based on entire coarse root systems (total), individual soil volume (ISV) or on fine roots (fine roots).

\begin{tabular}{llll}
\hline parameter & ranking of species & species separable (ANOVA) & remarks \\
\hline$D$ & S.c. $<$ G.f. $<$ S.s. $<$ V.i. & S.c. vs. V.i. & total \\
$D_{x y}$ & S.c. $<$ G.f. $<$ S.s. $<$ V.i. & S.c. vs. V.i. & total \\
$D_{x y}$ & V.i. $<$ G.f. $<$ S.s. $<$ S.c. & None & fine roots \\
$E(0)$ & S.c. $<$ S.s. $<$ G.f. $<$ V.i. & S.c. vs. V.i. & coarse roots \\
$E(0)$ & S.c. $<$ S.s. $<$ G.f. $<$ V.i. & V.i. vs. all, S.c. vs. G.f. & fine roots (inside \\
$E(0)$ & S.c. $<$ S.s. $<$ G.f. $<$ V.i. & V.i. vs. all & fine roots (outside \\
$E(\phi)$ & S.c. $<$ G.f. $<$ V.i. $<$ S.s. & None & ISV \\
$k$ & V.i. $<$ S.s. $<$ S.c. $<$ G.f. & V.i. vs. all & ISV $(\phi=2.5)$ \\
$E E$ & S.c. $<$ S.s. $<$ V.i. $<$ G.f. & G.f. vs. all, S.c. vs. V.i. & fine roots \\
$G E E(\phi)$ & S.c. $<$ S.s. $<$ V.i. $<$ G.f. & G.f. vs. all, S.c. vs. all & ISV \\
NEC & S.c. $<$ S.s. $<$ V.i. $<$ G.f. & None $(\phi) ~$ & IS $(\phi)$ \\
$R L D$ & S.c. $<$ S.s. $<$ G.f. $<$ V.i. & S.c. vs. V.i. & ISV \\
RLD & S.c. $<$ S.s. $<$ G.f. $<$ V.i. & V.i. vs. all & ISV \\
$q_{a}$ & S.c. $>$ S.s. $>$ G.f. $>$ V.i. & S.c. vs. all & total \\
$q_{b}$ & S.c. $>$ S.s. $>$ V.i. $>$ G.f. & S.c. vs. all & total \\
$I_{e}$ & S.s. $>$ S.c. $>$ G.f. $>$ V.i. & S.c. $\&$ S.s. vs. G.f $\&$ V.i. & total \\
$I_{i}$ & G.f. $>$ S.c. $>$ S.s. $>$ V.i. & None & total \\
\hline
\end{tabular}




\section{Supplementary comparisons}

Digitising and computer-based analysis techniques of whole coarse root systems proved to be very advantageous to obtain valuable 3D information. With the help of this database, useful information was obtained in order to analyse fractal dimensions, branching patterns, scaling relations and also explorative and exploitative features of the investigated species. It was possible to quantify distinctive features of the coarse root architecture and to describe species-dependent rooting behaviours. Additionally, data about fine root cores, which also contained spatial information, complemented the research. The combination of both data sets, completely excavated coarse root systems with spatial data from systematic fine root sampling, therefore revealed useful insight into details about entire root system structure. This method was suitable in order to distinguish species related differences, used to evaluate potential competition of the investigated species.

With the different quantitative methods it was possible to separate clearly contrasting rooting strategies within a plant community of co-existing species. This result confirms the assumption that species with complementary and dissimilar rooting architecture compete less and thus become preferred neighbours (Rundel and Nobel 1991). In addition it also confirms the view of other investigations, that distinct plant life-forms utilize different soil moisture input (Cohen 1970, Noy-Meir 1973, Cody 1986, Smith and Nobel 1986). For over 90 years this hypothesis has been supported by data comparing root distribution and water relations of co-occuring species in the same habitat (Canon 1911, Walter and Stadelmann 1974, Smith et al. 1997).

Throughout the spectrum of considered parameters (fractal dimension, topology, exploration and exploitation), the results showed a consistent picture concerning the ranking of the species. Particularly, S. cocculoides always turned out to be the 'weakest' conspecific in comparison to Grewia flava or Vangueria infausta. However, both last mentioned species sometimes rival with each other for the position as the 'strongest' competitor, especially, when aspects of exploration or exploitation are considered (Publication I - IV). But comparisons between different indices, sometimes revealed divergent patterns for the single species which were not easy to interprete.

Characteristics of fine root samples for the considered species have underlined that 'common strategies' exist between coarse and fine roots within the same species. It seems that a general construction plan, initiated in the development of fine roots, continues through the ontogeny of the indiviudals. But other, contrasting patterns between fine and coarse roots (e.g. mean values for $D_{x y}$ for fine roots and mean 
values for $D$ of coarse roots) indicate changes of the rooting patterns with increasing age.

Detailed information about different aspects, including fractals, topology, exploration and exploitation characteristics, are outlined in Publications I - IV. An integrating overview of the above mentioned subjects is given in the subsequent paragraphs with cross-correlations between the parameters from different chapters.

\subsection{Fractal dimension and Topology}

The relationship between topological parameters $\left(q_{a}\right.$ and $\left.q_{b}\right)$ and the box counting dimension $D$ for the coarse roots did not reveal strong results. However, the comparison did show better results as that with their corresponding unnormalized parameters altitude a and mean topological depth $b$. Thus the normed parameters seem to be more reliable than the classical topological parameters. The negative correlation clearly indicates that less intensively space filling root structures, i.e. herringbone patterns, characterized by higher values of the topological indices, were assigned to individuals with lower $D$ values.

Further negative trends were obtained comparing the parameters external $\left(l_{e}\right)$ and internal link lengths $\left(l_{i}\right)$ with the box counting dimension $D$. As link lengths are closely related with topological patterns, this result is not surprising, too, as a more herringbone configuration is typically characterised by longer external links (indicating less branching intensity) and this feature is accompanied by lower values of $D$. However, it has to be emphasised that the above described trends are not associated with tight correlations. They point at trends which can be explained with the nature of the parameters, but fractal dimension as well as the topological indices show a considerable amount of variation which cannot be explained by other parameters and are thus clearly to be regarded as independent variables, measuring different features of spatial architecture of root systems.

Not surprisingly the magnitude $\left(v_{0}\right)$ as well as the total number of links $(v)$ show a positive, though not very strong, correlation when compared with $D$.

Conscious of the fact that the box counting dimension ( $D$ or $D_{x y}$ ) only yields condensed information, it was possible to statistically separate the more intensively space filling species Vangueria infausta from Strychnos cocculoides. This contrasting position of both species was maintained in the majority of the cases, independent of other considered parameters. 


\subsection{Fractal dimension and Exploration}

\subsubsection{Coarse roots}

Comparisons between the fractal characteristics of the whole root systems (all species and individuals were pooled) with the exploration index $E(0)$ of the coarse roots revealed an interesting relationship. Although the box counting dimension $D$ was calculated for whole root systems, regression results between $D$ and the exploration index $E(0)$ were slightly better when a rooting depth between $0-80 \mathrm{~cm}\left(r^{2}\right.$ $=0.43$, respectively $0-60 \mathrm{~cm}: r^{2}=0.48$ ) was considered than for the entire root systems $\left(r^{2}=0.41\right)$. Similar results were obtained when $D$ and $R L D$ were compared. Here too, coefficients of determination were slightly better for considerations of the ISV $\left(r^{2}=0.48\right)$ than for entire root systems $\left(r^{2}=0.46\right)$. However, considering each single species, a distinct and persuasive positive trend was only found for Strychnos cocculoides $\left(r^{2}=0.85\right)$ and Vangueria infausta $\left(r^{2}=0.54\right)$. Unincisive correlations were obtained for samples from Strychnos spinosa $\left(r^{2}=0.05\right)$ and Grewia flava $\left(r^{2}=\right.$ 0.03 ), the latter showing even a negative trend. But these results corroborate once again that with considerations for a restricted soil volume, whole root systems can be adequately represented when the restricted soil volume is calculated for an appropriate rooting depth.

No connection was found between $D$ and other explorative (NEC, EE) or exploitative $(E(\phi), G E E(\phi))$ parameters of the coarse root systems, independent whether the entire root systems or only the ISV was considered. A conclusion was drawn that the box counting dimension $D$ can be a useful, but only rough estimator of complexity of spatial structures, that fails to quantify distinctive rooting architectures and therefore also lacks the ability to exactly predict possible differences in the potential competition.

\subsubsection{Fine roots}

Surprisingly, a comparison between mean values for the fractal dimensions from fine roots $\left(D_{x y}\right)$ did show a clear inverse trend with mean values from $E(0)$ obtained from fine roots. This indicates that species with highest values of $D_{x y}$, implying a more intensive space filling character, were least explorative, whereas high values of $E(0)$ seem to be represented by fine root samples with lower values for the box counting dimension. As $E(0)$ is defined as the proportion of soil volume that contains any roots, species with low percentage seem to develop fine roots with more intensively space filling patterns (expressed as $D_{x y}$ ), in order to gain maximum resource input from a restricted soil volume. With other words, species which can afford high expenditure do not 'care' about an intensive and thorough use of the resources, 
expressed as less space filling fine root arrangement (i.e., lower $D_{x y}$ values). This result might also coherently explain the distinct distribution of fine roots in relation to the vicinity of the coarse roots (Publication IV). More explorative species (e.g. Vangueria infausta and Grewia flava) can afford to scatter their numerous fine roots within a larger distance from the neighbouring coarse roots than less explorative species with additionally low amount of fine root containing cores.

\subsection{Topology and Exploration}

Mean values from $E(0)$ for the coarse root systems (ISV) of the single species performed strongly with an inverse correlation with mean values of $q_{a}\left(r^{2}=0.82\right)$. This result points to the fact, that herringbone branching patterns (with high values of $q_{a}$ ), in contrast to dichotomous ramification, are closely related with low exploration ability. In other words, in terms of coarse root distribution the most explorative species Vangueria infausta yielded lowest values for $q_{a}$, whereas individuals from the least explorative Strychnos cocculoides had highest mean values of $q_{a}$. Here too, values for $q_{a}$ from Grewia flava were similar to those of Vangueria infausta, indicating a more dichotomous structure, whereas values for Strychnos spinosa, once again, indidcate an intermediate type.

Another interesting aspect is the comparison between external link length $\left(I_{e}\right)$, a metric parameter, but closely related to topological distinctions, and $E(0)$. Here too, a negative correlation between the mean values for the single species is obvious. And this trend stands in close agreement with the common assumptions about topological features: lower values of $E(0)$ which are assigned to the herringbone type with typically longer external links stand in contrast to the characteristic dichotomous pattern.

All other topological parameters did not reveal any interesting behaviour when compared with the results of the exploration index from coarse roots.

\subsection{Exploration and other parameters}

\subsubsection{Coarse roots}

A comparison between $E(0)$ and $R L D$ of coarse root systems yielded, independent of the species, a clear linear relationship with good regression results $\left(r^{2}=0.99\right)$. Similar results were also obtained when different layers of rooting depths were considered. However, this result is not surprising but clearly indicates that, independent of the species and the occupied soil volume, the amount of cubes containing any roots is a quite good approximation of the sum of root lengths. 
The relation between exploration and exploitation regarding coarse and fine roots is thoroughly discussed in Publication IV.

\subsubsection{Fine roots}

Values from $E(0)$ for the fine roots were for all of the investigated species similar in ranking with the exploration index from coarse roots. This result indicates that a 'common strategy' between both entities can be assumed. In addition, the relationship between the exploration of fine roots with other parameters anaylsed are outlined in Publication IV. 


\section{References}

Berntson G.M. 1994. Modelling root architecture: are there tradeoffs between efficiency and potential of resource acquisition? New Phytol. 127: 483-493.

Berntson G.M. 1996. Fractal geometry, scaling, and the description of plant root architecture. In: Waisel Y., Eshel A., Kafkafi U. (eds.), Plant Roots: The Hidden Half, Dekker, New York, $2^{\text {nd }}$ ed., pp. 259-272.

Bohrer G. 2001. Evidence of host and environmental preferences in wild communities of VAM fungi in the Kalahari Desert. A thesis for the Masters degree, Department of Life Sciences, Faculty of Natural Sciences, Ben Gurion University, Israel. 67p.

Bohrer G., Kagan-Zur V., Roth-Bejerano N. and Ward D. 2001. Effects of environmental variables on vesicular-arbuscular mycorrhizal abundance in wild populations of Vangueria infausta. J. Veg. Sci. 12(2): 279-288.

Cannell M.G.R., van Noordwijk M. and Ong C.K. 1996. The central agroforestry hypothesis: the tree must aquire resources that the crop would not otherwise acquire. Agrofor. Syst. 34: 27-31.

Canon W.A. 1911. The Root Habits of Desert Plants. Carnegie Institution of Washington, Publication No. 131, Washington D.C.

Cohen D. 1970. The expected efficiency of water utilization in plants under different competition and selection regimes. Israel J. Bot. 19: 50-54.

Cody M.L. 1986. Structural niches in plant communities. In: Diamond J. and Case T.J. (eds), Community Ecology, Harper \& Row, New York, pp. 381-405.

De Costa W.A.J.M. and Chandrapala A.G. 2000 a. Competition between six hedgerow tree species and mung bean (Vigna radiata (L.) Wilczek) in the midcountry intermediate zone. J. Nat. Sci. Found. Sri Lanka 28(2): 113-125.

De Costa W.A.J.M. and Chandrapala A.G. 2000 b. Effects of tree root competition on availability of soil and plant nutrients, soil water and light interception in hedgerow intercrops with different tree species in the mid-country intermediate zone. J. Nat. Sci. Found. Sri Lanka 28(2): 127-142.

FAO. 1983. Food and Fruit-Bearing Forest Species-Examples from East Africa. Forestry Paper 44/1. Rome.

Fitter A.H. 1985. Functional significance of root morphology and root system architecture. In: Fitter A.H., Atkinson D., Read D.J. and Usher M.B. (eds), Ecological Interactions in Soil, Special publication of the Britsch Ecological Society, No. 4. Blackwell Scientific, Oxford, pp. 87-106. 
Fitter A.H. 1987. An architectural approach to the comparative ecology of plant root systems. New Phytol. 106 (Suppl.): 61-77.

Fitter A.H., Stickland T.R., Harvey M.L. and Wilson G.W. 1991. Architectural analysis of plant root systems 1. Architectural correlates of exploitation efficiency. New Phytol. 118: 375-382.

Fox F.W. and Norwood Young M.E. 1982. Food from the Veld. Edible Wild Plants of Southern Africa. Delta Books, Johannesburg, South Africa. 399p.

Gersani M., Brown J.S., O'Brian E.E., Godfrey M.M. and Abramsky Z. 2001. Tradegy of the commons as a result of root competition. J. Ecol. 89: 660-669.

Giess W. 1985. The naming and utilization of plantlife by the Ju//'hasi Bushmen of the Kau-Kauveld, In: Giess W. and Snyman J.W. (eds), Contemporary studies on Khoisan, vol. 1, pp. 237-347.

Hughes K.A., Gandar P.W. and de Silva H.N. 1995. Exploration and exploitation of soil by apple, kiwifruit, peach, Asian pear and grape roots. Plant Soil 175: 301-309.

Kaye B.H. 1989. A Random Walk Through Fractal Dimensions. VCH, Weinheim.

Mandelbrot B.B. 1977. The Fractal Geometry of Nature. Freeman, New York.

Mandelbrot, B.B. 1989. Multifractal measures, especially for the geophysicist. Pure Appl. Geophys. 131: 5-42.

Mark D.M. and Aronson P.B. 1984. Scale-dependent fractal dimensions of topographic surfaces: An empirical investigation, with applications in geomorphology and computer mapping. Math. Geol. 16: 671-683.

Mateke S.M. 2001. The effects of shade on initial growth, development and occurrences of leaf diseases on wild indigenous fruit trees Sclerocarya birrea and Strychnos cocculoides in shade netting conditions. In: Pasternak D. and Schlissel A. (eds), Combating Desertification with Plants, pp. 223-240.

McMahon T.A. and Kronauer R.E. 1976. Tree structures: deducing the principle of mechanical design. J. Theor. Biol. 59: 443-466.

Mphinyane W.N. 2001. Influence of livestock grazing within piospheres under free range and controlled conditions in Botswana. Doctoral Thesis. Faculty of Natural and Agricultural Science, Department of Plant Production and Soil Science, University of Pretoria. $125 p$.

Msanga H.P. and Maghembe J.A. 1989. Physical scarification and hydrogen peroxide treatments improves germination of Vangueria infausta seed. For. Ecol. Man. 28: 301-308. 
Noy-Meir I. 1973. Desert ecosystems: environment and producers. Ann. Rev. Ecol. System 4: 25-51.

Ong C.K., Corlett J.E., Singh R.P. and Black C.R. 1991. Above and below ground interactions in agroforestry systems. For. Ecol. Man. 45: 45-57.

Palgrave C.K. 1977. Trees of Southern Africa. $2^{\text {nd }}$ edition. Struik Publishers, Cape Town, South Africa. 959p.

Palmer E. and Pitman N. 1972. Trees of Southern Africa. Covering all known indigenous species. Volume 2 \& Volume 3. Balkema, Cape Town, South Africa.

Rao M.R., Ong C.K., Pathak P. and Sharma M.M. 1991. Productivity of annual cropping and agroforestry systems on a shallow Alfisol in semi-arid India. Agrofor. Syst. 15: 51-63.

Regional Soil Conservation Unit (RSCU). 1992. A Selection of Useful Trees and Shrubs for Tanzania. Draft. Nairobi.

Rundel P.W. and Nobel P.S. 1991. Structure and function in desert root systems. In: Atkinson D. (ed), Plant Root Growth - An Ecological Perspective, special publication number 10 ot the British Eoclogical Society, Blackwell Scientific, Oxford, pp. 349-378.

Schurr F. 2001 Grewia flava in the southern Kalahari. The population dynamics of a savanna shrub species. Diplomarbeit, Institut für Ökologie der Friedrich-SchillerUniversität Jena, Germany. 84p.

Schwinning S. and Ehleringer J.R. 2001. Water use trade-offs and optimal adaptations to pulse-driven arid ecosystems. J. Ecol. 89: 464-480.

Sing R.P., Ong C.K. and Saharan N. 1989. Above and below ground interactions in alley-cropping in semi-arid India. Agrofor. Syst. 9: 259-274.

Smith S.D., Monson R.K. and Anderson J.E. 1997. Physiological Ecology of North American Desert Plants. Springer-Verlag, Berlin, Germany. 286p.

Smith S.D. and Nobel P.S. 1986. Deserts. In: Baker N.R. and Long S.P. (eds), Photosynthesis in Contrasting Environments, Elsevier, Amsterdam, pp. 13-62.

Soil Survey Staff. 1999. Soil Taxonomy A Basic System of Soil Classification for Making and Interpreting Soil Surveys, 2nd. Edition. USDA (United States Department of Agriculture), NRCS (Natural Resources Conservation Service) Agriculture Handbook No. 436, Washington D.C. 871p.

Spek L.Y. and van Noordwijk M. 1994. Proximal root diameter as predictor of total root size for fractal branching models. II. Numerical model. Plant Soil 164: 119-127.

Story R. 1958. Some Plants Used by the Bushmen in Obtaining Food and Water. Mem. Bot. Survey S. Afr. 30: 1-115. 
Tardieu F., Bruckler L. and Lafolie F. 1992. Root clumping may affect the root water potential and the resistance to soil-root water transport. Plant Soil 140: 291301.

Taylor F.W. 1986. The potential for utilization of indigenous plants in Botswana. In: Wickens G.E., Goodin J.R. and Field D.V. (eds.), Plants for arid lands, George Allen and Unwin, London, pp. 231-242.

Taylor F. and Moss H. 1982. The Potential of Commercial Utilisation of Veldproducts in Botswana. Report for the Ministry of Commerce and Industry, Gaborone, Botswana.

Taylor F.W., Butterworth K.J and Mateke S.M. 1995. The importance of indigenous fruit trees in semi-arid areas of southern and eastern Africa. African Academy of Sciences Second Roundtable Discussions on Non-Timber Products, Pretoria, South Africa.

van Noordwijk M., Spek L.Y. and de Willigen P. 1994. Proximal root diameter as predictor of total root size for fractal branching models. I. Theory. Plant Soil 164: 107-117.

v. Koenen E. 1996. Heil- Gift- u. essbare Pflanzen in Namibia. Klaus Hess Verlag. $335 p$.

Walter H. and Stadelmann E. 1974. A new approach to the water relations of desert plants. In: Brown R. (ed), Desert Biology Volume 2, Academic Press, New York, pp. 213-310.

Wehmeyer A.S. 1966. The nutrient composition of some edible wild fruit found in the Transvaal. South Africa Med. J. 40: 1102-1104. 


\section{Acknowledgements}

Writing this thesis would have been impossible without the support of a number of people and their diverse helpful contributions.

Special thanks to my supervisor, Prof. Dr. D. L. Godbold, who inspired my interest in the subject of root research and provided numerous ideas that helped to establish the basic principles for the field work. His professional feedback, given across a large geographical distance, was always important for the progress and guidance of the project. In this connection, I would also like to acknowledge the aid of Dr. G. Jentschke who made useful suggestions with regard to the experimental design and the administrative frame of the project.

Furthermore, special thanks are due to Prof. Dr. W. Kurth who always supported this thesis with many new ideas and innovative energy, encouraging me and introducing me to some principles of accepted theories which helped to improve the present thesis. His personal input and also the support of $\mathrm{H}$. Dzierzon in the field of software adaptation deserve special recognition. Grateful thanks also to M. Götzie for his aid and assistance with the decoding of raw data and converting it into the required format suitable for further analysis.

The field assistance provided by Mmoluki Botite and Golekwang Piri was invaluable without it, the data could not have been obtained within the given time frame. Their special patience and resourcefulness in dealing with unexpected events often encouraged me to continue with the research work.

I am also deeply grateful to F. and M. Taylor for very special moral and technical support which created a very supportive atmosphere facilitating the work. Furthermore, all aid, support and knowledge that I received from the staff of Veld Products Research \& Development, especially the experience of S. Mateke on fruit trees, was essential for the progress of the work and deserves special acknowledment.

Funding for this research project was provided by the European Community under research grant INCO-DC (ERBIC18CT960035) and is hereby gratefully acknowledged. In this context, also the support from E. Bonifacio and the encouragement from $\mathrm{G}$. Bohrer are especially appreciated.

Furthermore, special thanks to A. Schreiner and C. Sarembe for their aid in translation and proof-reading the main parts of this thesis.

Last but not least, a special thankyou to all the people who have supported me throughout this project with their patience, advice and good humour - you know who you are. 

The following article

Structure and fractal dimension of root systems of four co-occuring fruit tree species from Botswana

contributed by:
A.L. Oppelt,
W. Kurth,
H. Dzierzon,
G. Jentschke,
D.L. Godbold

was published in:

Annals of Forest Science 57 (2000)

on pages:

$463-475$ 



\title{
Structure and fractal dimension of root systems of four co- occurring fruit tree species from Botswana
}

\begin{abstract}
Coarse root systems of four different fruit tree species from southern Africa were completely excavated and semi-automatically digitized. Spatial distributions of root length were determined from the digitally-reconstructed branching systems. Furthermore, the fractal characteristic of the coarse root systems was shown by determining the box-counting dimensions. These quantitative methods revealed architectural differences between the species, probably due to different ecophysiological strategies. For fine root samples, which were taken before digging out the whole systems, fractal analysis of the planar projections showed no significant inter-species differences. Methodologically, the study underlines the usefulness of digital 3-D reconstruction in root research.
\end{abstract}

\section{Introduction}

Investigations of root structure of tropical tree species are few. However, information on the rooting structure of locally and economically important species can be of great benefits. This is especially the case for fruit trees, where knowledge of root structure can provide a solid basis for sustainable use and integration into agriculture.

Qualitative architectural analysis was first developed for tree crowns by Hallé et al. [22]. Similar work on roots, aiming at an understanding of root system architecture as an indicator of growth strategy, was initiated by several authors, e.g. [18, 20]; see [5, $6,11,13,17,23]$ for studies on root systems of conifers. Morphological studies on root systems of angiosperms of temperate regions, e.g. [32], and on palms [24, 26] have also been carried out. Digitizing and computer-based analysis techniques can considerably improve the efficiency and reproducibility of such investigations (e.g. [3, $7,25]$ ). Numerous dynamic models of root growth have been developed on these grounds in the past $[4,8,9,31,36]$.

Various attempts have been made in the literature to quantify aspects of order in the growth forms of vegetation. One approach, dating back to the seminal work of Mandelbrot [33], determines the fractal dimension of a given morphological structure in space. Provided the fractal dimension is well-defined for the structure under consideration, it serves as a measure of occupation of space at different length scales and as an indicator of certain forms of self-similarity (see [14] and [16] for mathematical details). Besides its application to various abiotic structures [1], the concept of fractal dimension has been applied to above-ground branching patterns of 
trees $[41,44,45,40,29]$, to rhizomatous systems [40] and to root systems of several plant species. Berntson [2] gives a review of the attempts to determine fractal dimensions of root systems. Beginning with Tatsumi et al. [42], Berntson [2] notes that in all reviewed work dealing with real root structures (not just with abstract growth models), dimension estimation was only applied to planar projections of the root systems. Eshel [15] was to our knowledge the first to determine a fractal dimension $d$ of a complete root system embedded in full 3-D space. He made equidistant gelatin slices of a single root system of the dwarf tomato Lycopersicum esculentum and used image processing and manual box counting to estimate $d$ for the full system and for horizontal and vertical intersection planes.

For our sample root systems from four different tree species, we use a technique for complete digital reconstruction of 3-D branching systems. With such a "virtual tree" [38], all sorts of topological and geometrical analysis, amongst them fractal dimension estimation, can be carried out with ease. Similar digital plant reconstructions have been realized for the above-ground parts of a walnut tree [39] and for the root system of an oak tree [12]. Godin et al. [19] also develop techniques for encoding, reconstructing and analyzing complete 3-D plant architectures. In our study, 3-D reconstruction of root system architecture serves as a means to identify and to quantify differences in the rooting behaviour of four different species growing under similar environmental conditions. We present only a small subset of the possible analysis options available for digital plant reconstructions; much more can be done.

\section{Materials and Methods}

\subsection{Site description}

The investigation site is located on sandveld near Mogorosi (Serowe Region, Central District, Botswana) between longitude $26^{\circ} 36.26^{\prime}$ and $26^{\circ} 36.70^{\prime} \mathrm{E}$ and latitude from $22^{\circ} 25.09^{\prime}$ to $22^{\circ} 25.30^{\prime} \mathrm{S}$. The vegetation can be described as bushveld with Acacia spp. and Terminalia spp. as characteristic species. The soils, mainly originating from sandstones, can be, according to the USDA-Soil Taxonomy, characterised as poorly developed Entisols. A very low fertility status, especially in organic carbon, even in the surface horizon, and low iron contents are characteristic in that region. The low amount of rainfall [10], which is about $430 \mathrm{~mm} / \mathrm{yr}$, and, additionally, high evapotranspiration, also during the growing season, are responsible for low crop yields. 


\subsection{Recording of root structure}

The architecture of in situ grown coarse root systems of the fruit tree species Strychnos cocculoides [Loganiaceae] (Mogorogorwane), Strychnos spinosa (Morutla) and Vangueria infausta [Rubiaceaea] (Mmilo) as well as from the shrub Grewia flava [Tiliaceae] (Moretlwa) was studied. "Coarse roots" were defined by a diameter $\geq 3$ $\mathrm{mm}$. For roots below this threshold, a reconstruction of spatial orientation and branching would not have been possible with our method.

Five coarse root systems of each species were investigated. The whole coarse root systems were excavated by manual digging. After exposure of the roots, they were divided and permanently marked with white ink into segments of different length, at each point where growth direction changed. The vertical angle and the magnetic bearing (azimuth) of each segment was determined and their individual length was recorded by a digital compass (TECTRONIC 4000, Breithaupt, Kassel - Germany) creating an ASCII-file (L-file).

After measurement of the original position in the field, the coarse roots were removed and the diameters of each single segment of the complete root system measured with a digital caliper (PM 200, HHW Hommel - Switzerland) creating a corresponding file (D-file) to each L-file.

Both raw data sets ( $\mathrm{L}$ - and D-files) were merged by an interface software creating the final code for reconstruction. For encoding the full geometrical and topological structure of the root systems (lengths, orientations and diameters of all segments and mother-segment linkages) we used the $d t d$ code (digital tree data format [28, 30]). The dtd files, each representing one complete root system, were generated semiautomatically as described above, and served as input for the software GROGRA 3.2 $[28,29]$ for $3-D$ reconstruction of the excavated systems.

The GROGRA software is suitable for reconstruction of a three dimensional topological and geometrical structure, so that a visual comparison between reality in the field and the generated description files from measurements can be obtained. Furthermore, GROGRA provides several algorithms for different types of analysis of 3-D branching structures (determination of root density in given spatial grids, fractal dimension, tapering, classification according to branching order). Here we concentrate on overall root system structure and on fractal analysis; an investigation of tapering and cross-section areas will be presented in a forthcoming paper.

To describe the internal topology of the branching systems, a developmental topological concept of branching order was used: The order of the tap root (if it exists) is 0 , and an $n$-th order root has branches of order $n+1$. The branching order was calculated for each segment automatically by GROGRA. 


\subsection{Fine roots}

Before excavation of the coarse roots, systematic fine root sampling was carried out around every tree with a soil auger ( $\varnothing 80 \mathrm{~mm}$, volume 1 litre). Ninety six samples per tree were collected. Core samples were taken on the cross points between three concentric circles $(r=1,2,3 \mathrm{~m})$ with eight centripetal lines (N, NE, E, SE, S, SW, W and NW) in four depths $(0-20,20-40,40-60$ and $60-80 \mathrm{~cm})$. Roots were removed by dry sieving and separated from roots originating from other species. For morphological analysis, 15 fine root samples per tree were chosen randomly and fixed in isopropanol. Fractal analysis of the fine roots was carried out using a flat bed scanner (HP 4Jc) with the software WinRHIZO 3.10.

\subsection{Fractal dimension analysis}

Fractal dimension can be conceived as a measure how intensely an object fills the space [1]. A value of 1 corresponds to a single Euclidean line, 2 to a planar object, and a value of, say, 1.5 characterizes an object filling "more" space than a line, but "less" than a plane. For practical purposes, the fractal dimension is commonly approximated by the box counting dimension $D$ [16]. $D$ is estimated by superimposing a mesh consisting of cubic boxes with length $s$ (= resolution or scale of the mesh), and by determining the number $N(s)$ of boxes containing a part of the object under consideration (Figure 1). This counting is repeated for a set of scales from a given range, and $D$ is obtained as the negative slope of the regression line in the log-log plot of $N(s)$ versus $s$ (e.g. [1]), see Figure 2.

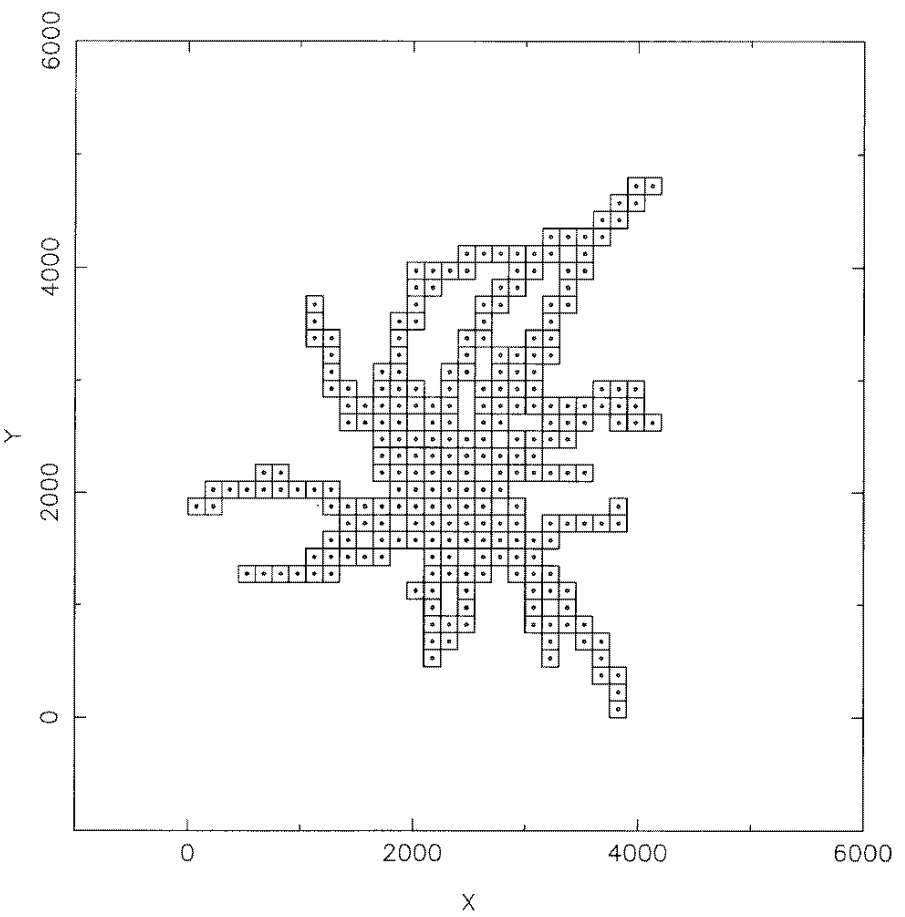

Figure 1. Projection of an analysed root system in the $150 \mathrm{~mm}$ resolution grid. 
For determining the regression line, we used unweighted least squares, though the data points in the box-count plot are not independent from each other and the statistical error will normally not be uniform in $s$. The obtained coefficients of determination $R^{2}$ are therefore usually higher than with independent data points; the models appear to fit better than they actually do [37, 43]. We nevertheless applied the simple least-squares procedure, since the determination of statistical precision of dimension estimators is a rather exacting task and was realized in the literature only under simplifying assumptions [21, 43]. Moreover, it is problematic to compare fractal dimensions estimated with different methods or obtained from different ranges of scales [16]. Hence our numerical results have to be relativized, but can give some information when compared with each other, since we used a fixed set of scales for all samples.

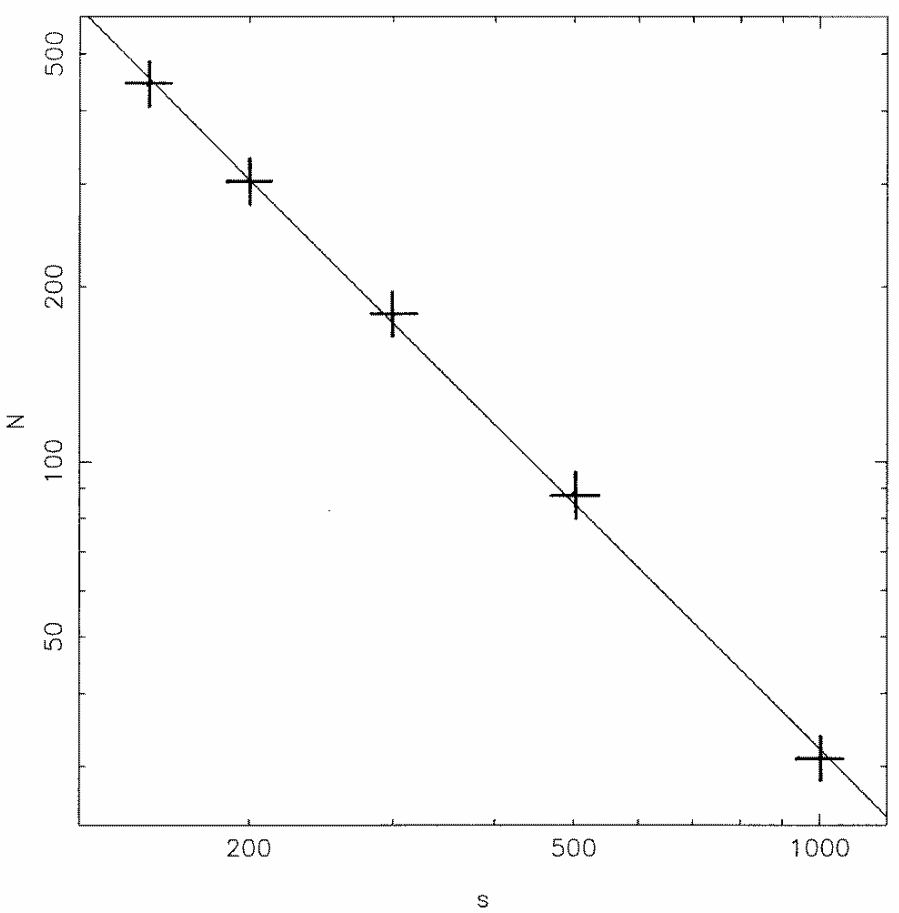

Figure 2. Doubly logarithmic plot of number of boxes (N) vs. resolution (s).

We have decided to use a lowest grid resolution of $1 \mathrm{~m}$, which falls just under the order of magnitude of the overall root system extension, and a highest resolution of $15 \mathrm{~cm}$, which lies in the order of magnitude of the mean interbranching distance occurring in our samples (cf. Table 1). Pretests have shown that when using even higher resolutions, the obtained dimension value becomes unstable and eventually falls to a value near 1 . Like for other natural phenomena, and in contrast to mathematically defined self-similar sets, a fractal behaviour can be obtained only within a limited range of scales [27]. Between the two extremes, we have added only three further resolution values $(50,30,20 \mathrm{~cm})$ to keep the amount of calculation time reasonable. A sensitivity analysis carried out at one of the samples showed no gain 
of precision of the obtained dimension value when 10 more intermediate resolutions were used - an observation which is in accordance with theory [21].

In addition to the box-counting dimension $D$ calculated on the basis of the 3-D reconstruction of the coarse root systems, we have determined the dimension $D_{x y}$ of the parallel projection of the whole system into a horizontal plane, using the same set of grid resolutions.

\subsection{Determination of tree age}

Tree age was estimated by counting the number of rings at the root collar. Root collar sections were carefully sanded and the number of rings counted using a binocular. As the Serowe area has only one rainy season per year, each ring was considered to be one year's growth.

\section{Results}

\subsection{General description of the root systems excavated}

Table 1 gives an overview on basic parameters of all the investigated root systems. All data were calculated with the help of GROGRA 3.2 [28].

Analysis of growth rings showed that the trees have a wide range of ages: Grewia flava (13-25 years), Strychnos cocculoides (13-29 years), Strychnos spinosa (12-20 years) and Vangueria infausta (19-36 years). As a consequence, parameters like the root collar diameter $(27-140 \mathrm{~mm})$, the total root length (11-207 $\mathrm{m})$, the extension in North-South-direction $(1.1-10.0 \mathrm{~m})$ as well as in West-East-direction $(0.7-10.1 \mathrm{~m})$ and the maximal radial extension $(1.2-6.0 \mathrm{~m}$ ) varied strongly between individuals in a species (see Table 1). Although all the excavated root systems represent different ages, species-dependent characteristics could be identified.

\subsection{Description of root systems}

To demonstrate the typical features, two root systems of each species with different ages were chosen. One lateral view (Figure 3 ) and one view of each root system from above (Figure 4) is shown. The lateral view shows the system from the south, i.e. west is on the left and east on the right hand side.

In Grewia flava coarse root systems (Figure 3), most of the first order laterals were almost horizontally orientated and concentrated in the upper soil layers, even when a deep tap root had developed. Long first order laterals showed downward growth only at distance from the shoot base. Vertical roots were mostly second or even higher order roots. Long tap roots were sometimes present but were not typical for a Grewia root system. Adventitious roots, originating from the shoot, were a typical feature of old Grewia flava root systems. 
Table 1. Basic parameters of individual root systems excavated.

\begin{tabular}{|c|c|c|c|c|c|c|c|c|c|c|}
\hline species & $\left.i^{1}\right)$ & $\begin{array}{l}\text { age } \\
\text { [yr] }\end{array}$ & $\begin{array}{c}\left.\mathrm{cd}^{2}\right) \\
{[\mathrm{mm}]}\end{array}$ & $\begin{array}{l}\left.\operatorname{trl}^{3}\right) \\
{[\mathrm{m}]}\end{array}$ & $\begin{array}{c}\left.r_{\max }^{4}\right) \\
{[\mathrm{m}]}\end{array}$ & $\begin{array}{c}\left.\mathrm{z}_{\max }^{5}\right) \\
{[\mathrm{m}]}\end{array}$ & $\left.b_{\max }{ }^{6}\right)$ & $\begin{array}{l}\left.\mathrm{ibd}^{7}\right) \\
{[\mathrm{mm}]}\end{array}$ & $\begin{array}{c}\left.\mathbf{r l d}^{8}\right) \delta \\
{\left[\mathbf{c m} / \mathbf{d m}^{3}\right]}\end{array}$ & $\begin{array}{c}\left.\mathrm{rvd}^{9}\right) \\
{\left[\mathrm{cm}^{3} / \mathbf{c m}^{3}\right]}\end{array}$ \\
\hline \multirow{5}{*}{$\begin{array}{l}\text { Grewia } \\
\text { flava }\end{array}$} & 203 & 25 & 97 & 191 & 5.7 & 2.3 & $3+a d$ & 656 & 0.0806 & 0.0035 \\
\hline & 206 & 21 & 65 & 73 & 3.3 & 2.6 & $3+a d$ & 459 & 0.0842 & 0.0033 \\
\hline & 213 & 18 & 58 & 59 & 3.3 & 3.2 & 3 & 554 & 0.0522 & 0.0016 \\
\hline & 214 & 14 & 32 & 20 & 1.6 & 2.0 & $3+a d$ & 183 & 0.1219 & 0.0032 \\
\hline & 215 & 13 & 27 & 8 & 1.5 & 2.0 & $2+a d$ & 33 & 0.0579 & 0.0019 \\
\hline \multirow{3}{*}{$\begin{array}{l}\text { mean } \\
\text { std } \\
\text { median }\end{array}$} & & 18 & 56 & 70 & 3.1 & 2.4 & & 377 & 0.08 & 0.003 \\
\hline & & 5.0 & 28.2 & 72.4 & 1.7 & 0.5 & & 174.1 & 0.03 & $8.810^{-4}$ \\
\hline & & 18 & 58 & 59 & 3.3 & 2.3 & & 459 & 0.08 & 0.003 \\
\hline \multirow{5}{*}{$\begin{array}{l}\text { Strychnos } \\
\text { cocculoides }\end{array}$} & 405 & 23 & 56 & 38 & 2.5 & 2.0 & 3 & 23 & 0.1011 & 0.0065 \\
\hline & 409 & 15 & 67 & 25 & 4.4 & 1.5 & 2 & 97 & 0.0263 & 0.0031 \\
\hline & 412 & 17 & 48 & 19 & 3.9 & 1.9 & 2 & 161 & 0.0207 & 0.0018 \\
\hline & 425 & 29 & 68 & 51 & 3.4 & 1.7 & 3 & 406 & 0.0814 & 0.0068 \\
\hline & 426 & 13 & 44 & 21 & 3.0 & 1.8 & 2 & 103 & 0.0403 & 0.0027 \\
\hline \multirow{3}{*}{$\begin{array}{l}\text { mean } \\
\text { std } \\
\text { median }\end{array}$} & & 19 & 57 & 31 & 3.4 & 1.8 & & 158 & 0.05 & 0.004 \\
\hline & & 6.5 & 10.9 & 13.5 & 0.74 & 0.2 & & 71.4 & 0.04 & $2.310^{-3}$ \\
\hline & & 17 & 56 & 25 & 3.4 & 1.8 & & 103 & 0.04 & 0.003 \\
\hline \multirow{5}{*}{$\begin{array}{l}\text { Strychnos } \\
\text { spinosa }\end{array}$} & 501 & 20 & 72 & 114 & 6.0 & 1.7 & 2 & 426 & 0.0577 & 0.0029 \\
\hline & 508 & 12 & 60 & 43 & 4.2 & 3.1 & 2 & 396 & 0.0248 & 0.0021 \\
\hline & 509 & 17 & 40 & 11 & 1.4 & 2.1 & 2 & 39 & 0.0764 & 0.0073 \\
\hline & 510 & 15 & 64 & 49 & 2.8 & 1.3 & 2 & 138 & 0.1595 & 0.0096 \\
\hline & 511 & 12 & 44 & 35 & 2.7 & 1.7 & 2 & 194 & 0.0880 & 0.0038 \\
\hline \multirow{3}{*}{$\begin{array}{l}\text { mean } \\
\text { std } \\
\text { median }\end{array}$} & & 15 & 56 & 50 & 3.4 & 2.0 & & 239 & 0.08 & 0.005 \\
\hline & & 3.4 & 13.6 & 38.4 & 1.8 & 0.7 & & 194.1 & 0.05 & $3.210^{-3}$ \\
\hline & & 15 & 60 & 43 & 2.8 & 1.7 & & 194 & 0.08 & 0.004 \\
\hline \multirow{5}{*}{$\begin{array}{l}\text { Vangueria } \\
\text { infausta }\end{array}$} & 706 & 36 & 140 & 207 & 5.1 & 2.5 & 5 & 309 & 0.1032 & 0.0062 \\
\hline & 708 & 21 & 86 & 52 & 3.6 & 2.4 & 4 & 178 & 0.0520 & 0.0030 \\
\hline & 711 & 19 & 48 & 25 & 1.7 & 1.3 & 3 & 142 & 0.2037 & 0.0106 \\
\hline & 712 & 25 & 60 & 25 & 1.2 & 1.8 & 4 & 120 & 0.2817 & 0.0123 \\
\hline & 713 & 25 & 70 & 35 & 1.5 & 1.1 & 4 & 252 & 0.4132 & 0.0177 \\
\hline \multirow{3}{*}{\multicolumn{2}{|c|}{$\begin{array}{l}\text { mean } \\
\text { std } \\
\text { median }\end{array}$}} & 25 & 81 & 69 & 2.6 & 1.8 & & 200 & 0.21 & 0.010 \\
\hline & & 6.6 & 35.9 & 78.2 & 1.7 & 0.6 & & 70.1 & 0.14 & $5.710^{-3}$ \\
\hline & & 25 & 70 & 35 & 1.7 & 1.8 & & 178 & 0.20 & 0.011 \\
\hline \multicolumn{6}{|c|}{$\begin{array}{l}\text { 1) Identifying number of individual } \\
2 \text { ) Root collar diameter } \\
\text { 3) Total root length } \\
\text { 4) Maximal radius of the whole root system } \\
\text { 5) Maximal depth of the whole root system } \\
6 \text { ) Branching order; ad: adventitious roots }\end{array}$} & $\begin{array}{l}\text { 7) Inter } \\
\text { orde } \\
{ }^{8} \text { ) Root } \\
9 \text { ) Root } \\
\text { (rvd }\end{array}$ & $\begin{array}{l}\text { anchi } \\
\text { ngth } \\
\text { lume }\end{array}$ & $\begin{array}{l}\text { distanc } \\
\text { sity (rl } \\
\text { nsity } \\
\text { volum }\end{array}$ & $\begin{array}{l}\text { mean of } \\
\operatorname{tr} l / \pi r_{\max }^{2} \\
\mathrm{~d}=\operatorname{tr} l / \pi r_{\mathrm{n}}\end{array}$ & $\begin{array}{l}\text { ax) } \\
\left.{ }^{2} z_{\max }\right)\end{array}$ \\
\hline
\end{tabular}

In contrast to the other investigated species, individuals from both Strychnos (Figure 3) species developed a pronounced and deeply penetrating tap root showing intensive secondary growth.

In the case of $S$. cocculoides the first order laterals were inserted along the whole tap root, with no obvious pattern of longer or shorter roots. Most of the long first order laterals are bow-shaped because of change in growth direction with time. 


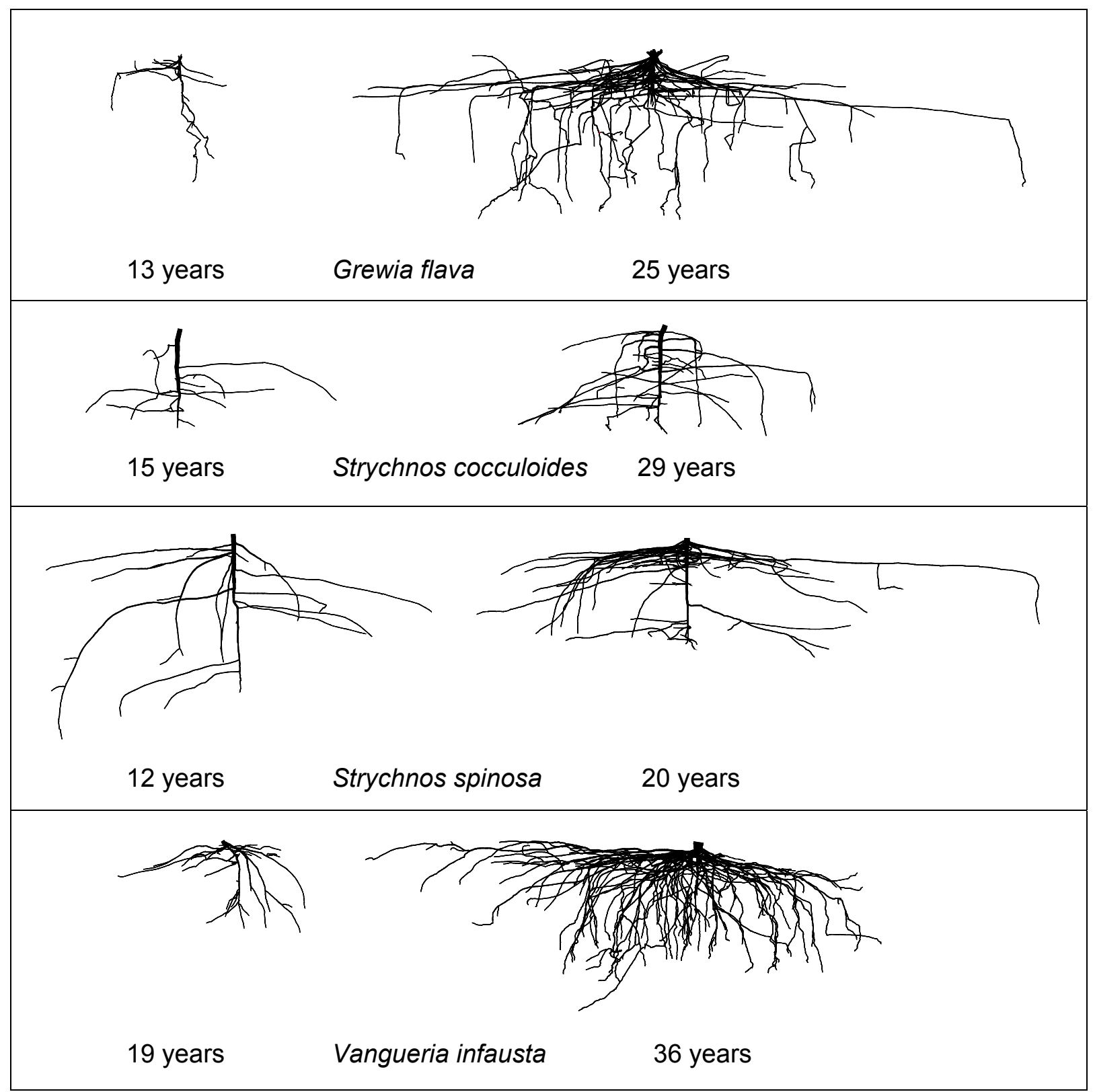

Figure 3. Lateral view of coarse root systems from Grewia flava, Strychnos cocculoides, Strychnos spinosa and Vangueria infausta in different age classes. Scale 1:100.

The typical Strychnos spinosa root architecture had similar features to that of $S$. cocculoides (intensive tap roots), but showed a strong decline in length of first order roots from upper to deeper soil layers, which clearly distinguishes both species. The branching intensity was extraordinarily low, as the coarse roots did not develop roots of higher branching order than 2 in all the excavated individuals.

In comparison to the above described species, the first order roots of Vangueria infausta showed a more plagiogeotropic growth direction. First order roots of Vangueria infausta are more frequently and intensively branched into second and higher order roots than the other species investigated. Coarse roots from this species develop a relatively dense network. 


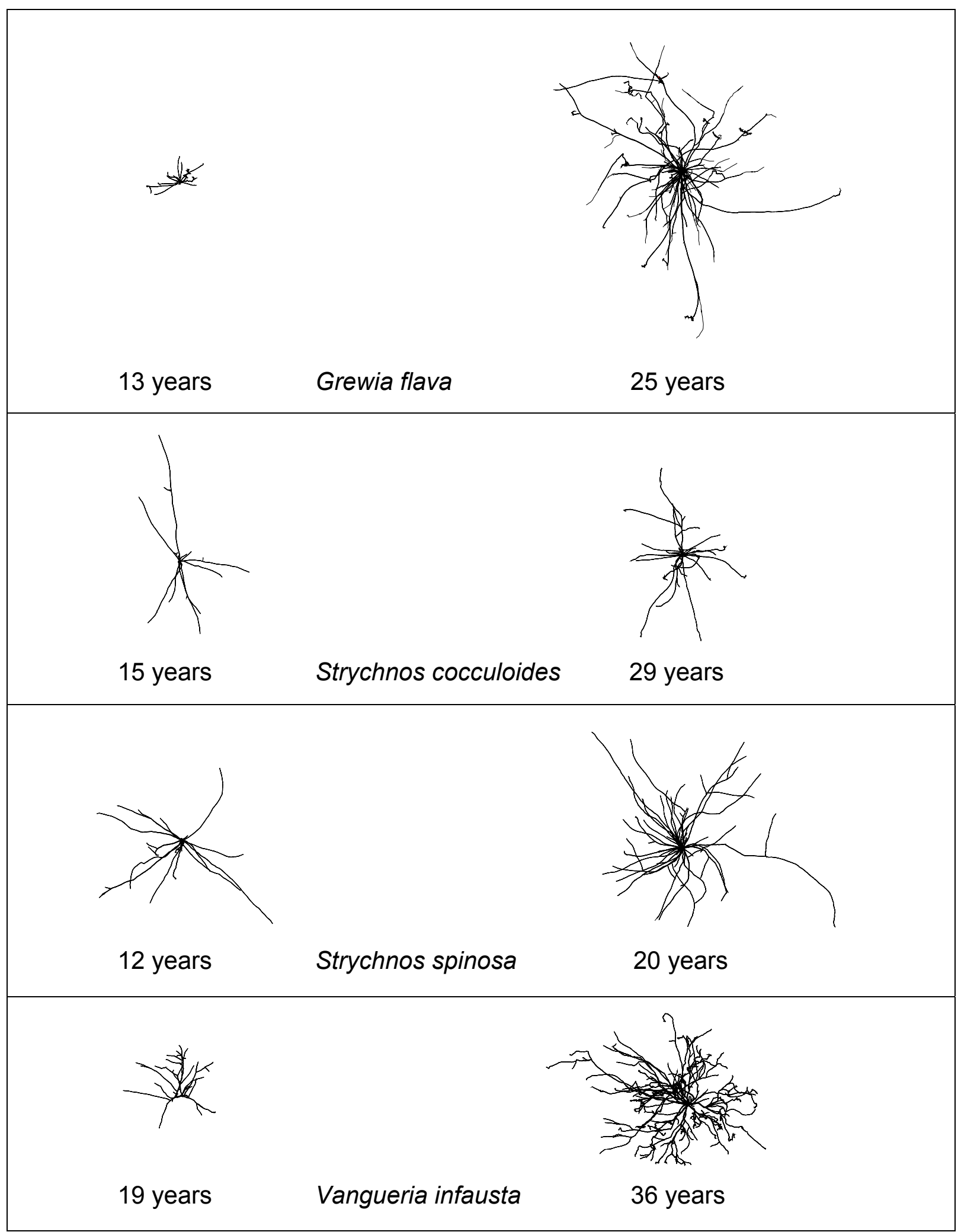

Figure 4. View from above of four coarse root systems from Grewia flava, Strychnos cocculoides, Strychnos spinosa and Vangueria infausta in different age classes. Scale 1:200.

We checked the tightness of correlation between age and other basic parameters. For the number of terminal roots, total root length and collar diameter, respectively, vs. age, clear linear dependencies governed the total population of investigated root 
systems $\left(R^{2}=0.58,0.42,0.61\right.$ respectively). When the fitting was done for each species separately, the correlation did normally increase ( $R^{2}$ between 0.63 and 0.98$)$, with the exception of Strychnos spinosa with a weak age dependence of the number of terminal roots and of total root length $\left(R^{2}=0.24\right.$, resp. 0.34), and with the exception of both Strychnos species in the case of collar diameter vs. age ( $S$. cocculoides: $R^{2}=0.33$, S. spinosa: $R^{2}=0.15$ ).

For other global parameters, like rooting depth and maximal radius of the system, as well as for root length density and mean interbranching distance, no clear age trend could be statistically identified in the total population, though some of these parameters were well correlated with age when only the representatives of one species were considered (Strychnos cocculoides: $R^{2}=0.61$ and 0.78 for root length density and root volume density increasing with age, resp., and in Grewia flava and Vangueria infausta: $R^{2}=0.77$, resp. 0.73 , for mean interbranching distance growing with age).

\subsection{Quantitative analysis of coarse root systems}

For quantifying distinctive features of the coarse root architecture and for description of species-dependent rooting behaviour, the horizontal and vertical distribution of the lengths of the coarse roots is shown (Figures 5 and 6 ). Since the different ages of the selected trees lead to a considerable variation of horizontal and vertical extension of the single root systems, all values are always expressed as percentage of the total root length of the whole system.

Although all the species differed in root architecture, radial distribution is quite similar in all four species. For all species a maximum of root length density was found near to the center, i.e. within $0.5 \mathrm{~m}$ distance from the trunk. However, a high variation was found between individuals of Grewia flava $\left(\operatorname{std}_{0.5 \mathrm{~m}}=28.3\right.$ ) and Vangueria infausta $\left(\operatorname{std}_{0.5 \mathrm{~m}}=22.6\right)$. Furthermore, both of these species show a second peak of variation at a distance of $1.5 \mathrm{~m}$ for Grewia flava $\left(\operatorname{std}_{1.5 \mathrm{~m}}=6.5\right.$ ) and at $2.0 \mathrm{~m}$ for Vangueria infausta $\left(\operatorname{std}_{2.0} \mathrm{~m}=28,3\right)$. In contrast, both Strychnos species have a more homogeneous radial distribution of coarse roots with a lower range of variation. This was more pronounced in Strychnos spinosa $\left(\operatorname{std}_{0.5 \mathrm{~m}}=12.4\right)$ than in $S$. cocculoides $\left(\operatorname{std}_{0.5} \mathrm{~m}=6.4\right)$, which shows the most homogeneous radial coarse root distribution $\left(\operatorname{std}_{\min }=0.3,350<d<400 \mathrm{~cm}\right.$ ) among the investigated species (Figure 5). 


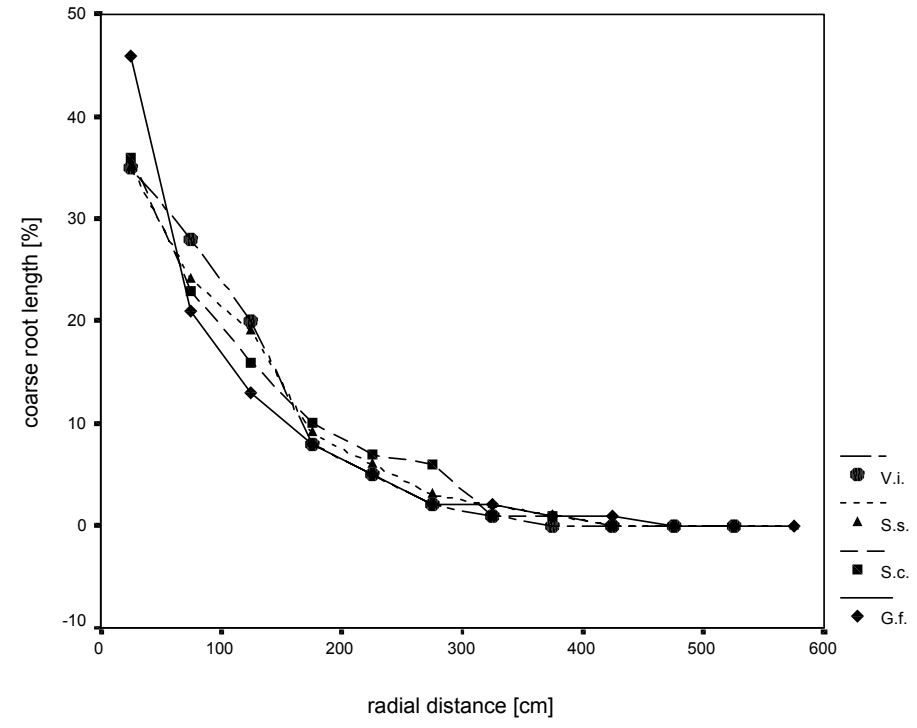

Figure 5. Radial distirbution of coarse roots from Grewia flava (G.f.), Strychnos cocculoides (S.c.), Strychnos spinosa (S.s.) and Vangueria infausta (V.i.).

Contrary to the radial distribution of the coarse roots, the vertical distribution shows marked differences between species. Wheras Grewia flava, Strychnos spinosa and Vangueria infausta reach their maximum root length in a depth of $40 \mathrm{~cm}$, Strychnos cocculoides exhibits a peak of coarse root density in a depth of $100 \mathrm{~cm}$ (Figure 6).

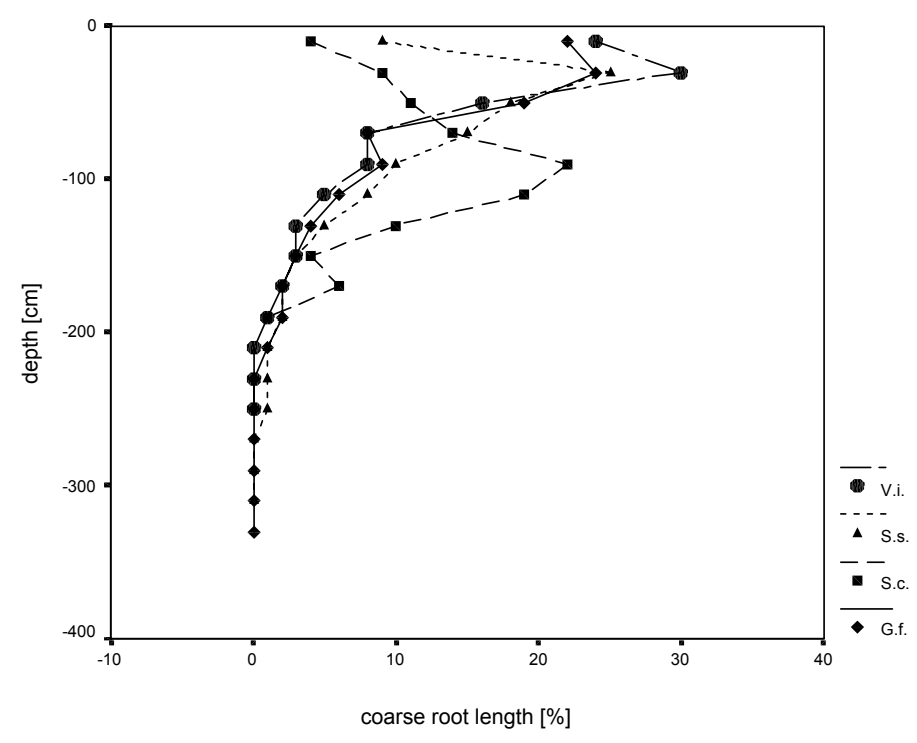

Figure 6. Vertical distribution of coarse roots from Grewia flava (G.f.), Strychnos cocculoides (S.c.), Strychnos spinosa (S.s.) and Vangueria infausta (V.i.).

\subsection{Fractal geometry}

\subsubsection{Coarse roots}

The fractal dimension was approximated by the box counting dimension $(D)$. The value of $D$ can range from 0 to 3 ; the value of 0 occurs for an empty space or for a point-like structure, whereas the value of 3 is obtained when a three dimensional space is completely filled. $D$ can be interpreted as a measure of the spatial distribution of coarse root systems. The results of dimension analysis are shown in Table 2. The values of $D$ are dependent on the chosen range of scaling (150-1000 
$\mathrm{mm}$ in all cases). The $R^{2}$ for the underlying log-log relation was in all cases around 0.99 , hence in the considered range of resolutions the root systems can be seen as fractals. The same holds for their projections into a horizontal plane ( $x y$-plane), with $D_{x y}$ as the resulting box counting dimension. Since the root systems differ considerably in their spatial extension, in the last column of Table 2 we show the number of boxes $N$ checked at highest resolution during the 3-D.

The mean value of the box counting dimension shows clear differences between Strychnos cocculoides with the lowest and Vangueria infausta with the highest $D$. This was confirmed by a one-factor ANOVA with subsequent least-significancedifference test, indicating a difference between these two species at the $5 \%$ level.

Table 2. Fractal analysis of the whole coarse root systems of four co-occurring species (3D).

\begin{tabular}{|c|c|c|c|c|}
\hline species & id $^{1}$ ) & $D$ & $D_{x y}$ & $\mathbf{N}$ \\
\hline \multirow{5}{*}{$\begin{array}{l}\text { Grewia } \\
\text { flava }\end{array}$} & 203 & 1.40 & 1.38 & 77452 \\
\hline & 206 & 1.46 & 1.45 & 14616 \\
\hline & 213 & 1.40 & 1.42 & 19712 \\
\hline & 214 & 1.28 & 1.34 & 4080 \\
\hline & 215 & 1.30 & 1.13 & 990 \\
\hline mean & & 1.37 & 1.34 & \\
\hline Std & & 0.08 & 0.13 & \\
\hline \multirow{5}{*}{$\begin{array}{l}\text { Strychnos } \\
\text { cocculoides }\end{array}$} & 405 & 1.50 & 1.35 & 7308 \\
\hline & 409 & 1.17 & 1.09 & 12375 \\
\hline & 412 & 1.26 & 1.28 & 11550 \\
\hline & 425 & 1.37 & 1.32 & 15624 \\
\hline & 426 & 1.22 & 1.22 & 12506 \\
\hline mean & & 1.3 & 1.25 & \\
\hline Std & & 0.13 & 0.1 & \\
\hline \multirow{5}{*}{$\begin{array}{l}\text { Strychnos } \\
\text { spinosa }\end{array}$} & 501 & 1.43 & 1.41 & 31464 \\
\hline & 508 & 1.24 & 1.28 & 30240 \\
\hline & 509 & 1.41 & 1.33 & 1456 \\
\hline & 510 & 1.55 & 1.52 & 6561 \\
\hline & 511 & 1.33 & 1.38 & 8004 \\
\hline mean & & 1.39 & 1.38 & \\
\hline Std & & 0.12 & 0.09 & \\
\hline \multirow{5}{*}{$\begin{array}{l}\text { Vangueria } \\
\text { infausta }\end{array}$} & 706 & 1.60 & 1.56 & 31212 \\
\hline & 708 & 1.33 & 1.43 & 12800 \\
\hline & 711 & 1.34 & 1.45 & 3420 \\
\hline & 712 & 1.62 & 1.39 & 2080 \\
\hline & 713 & 1.66 & 1.50 & 2448 \\
\hline mean & & 1.51 & 1.47 & \\
\hline Std & & 0.16 & 0.07 & \\
\hline
\end{tabular}

${ }^{1}$ ) Identifying number of individual

However, the different ages of our investigated coarse root systems could also influence $D$. When data from all species were considered in one analysis, the box counting dimension was found to correlate positively with age $\left(R^{2}=0.44\right)$ (Figure 7). 
This age-dependent increase of $D$ seems to be strongly apparent in Grewia flava ( $R^{2}$ $=0.63)$ and Strychnos cocculoides $\left(R^{2}=0.55\right)$ but less pronounced in Vangueria infausta $\left(R^{2}=0.41\right)$ and Strychnos spinosa $\left(R^{2}=0.30\right)$. An analysis of covariance with the species as factor and age as covariable indicated a significant positive influence of age on $D$. The box counting dimensions of Strychnos cocculoides and Vangueria infausta, now at the $1 \%$ level were significantly different.

However, in view of the low number of replicates for the individual species, further investigations will be necessary to fully assess the relationship between age and fractal dimension.

We tried to relate the box counting dimensions of the individual coarse root systems (Table 2) also to other global parameters characterizing the root systems (cf. Table 1). The correlation to $D$ was particularly strong in the case of root length density $\delta$ . (simply defined as total root length, divided by the volume of the smallest cylinder containing the root system), the coefficient of determination being $R^{2}=0.51$ when all individuals are considered together. Figure 8 shows that this relationship is even closer when only the two Strychnos species are considered separately, and that shape and tightness of the regressions differ considerably between the four species. Root volume density (sum of root segment volume divided by volume of containing cylinder) shows also a clear correlation to $D\left(R^{2}=0.52\right)$ for all 20 individuals taken together (diagram not shown), with similar differences between the species. For other global attributes (total length, maximal radius, maximal depth, collar diameter, mean interbranching distance) the relationships to $D$ are only weak ( $R^{2}$ between 0.01 and $0.20)$.

The box counting dimensions $D$ obtained from full 3-D analysis did not differ by more than 0.23 from the corresponding values $D_{x y}$ (mean: 0.03 , std: 0.09 ) from 2-D analysis of the projections in the $x y$-plane (Figure $9, R^{2}=0.57$ ). Statistically, the resulting regression line could not be separated from the angle bisector $D=D_{x y}(p=$ 0.25).

\subsubsection{Fine roots}

Table 3 shows results from the calculation of $D_{x y}$ for the projected fine roots. The used range of grid resolutions was from 0.05 to $3.0 \mathrm{~mm}$. Between the species, no significant differences in the $D_{x y}$ value for the fine roots are apparent.

Table 3. Fractal analysis of fine root samples (2-D).

\begin{tabular}{lccc}
\hline species & $\mathbf{N}$ & mean $\left(\mathbf{D}_{\mathbf{x y}}\right)$ & $\boldsymbol{s t d}\left(\mathbf{D}_{\mathbf{x y}}\right)$ \\
\hline Grewia flava & 35 & 1.42 & 0.14 \\
Strychnos cocculoides & 28 & 1.48 & 0.12 \\
Strychnos spinosa & 44 & 1.46 & 0.09 \\
Vangueria infausta & 59 & 1.41 & 0.06 \\
\hline
\end{tabular}




\section{Discussion}

The analysis suggests that the investigated species, although growing under the same environmental conditions, have different rooting strategies which are expressed in the architectures of the coarse root systems.

Both Strychnos species show a tendency towards deep rooting behaviour. The ecophysiological advantage may be to obtain access to water deep in the soil profile.

The development of coarse root systems in Grewia flava is initially shallow. We suggest that Grewia flava may be able to utilize periodic rain fall, especially low quantities, before the water is evaporating from the soil. Additionally, this feature aids a better nutrition supply from the organic upper horizons. Vangueria infausta shows an intermediate strategy.
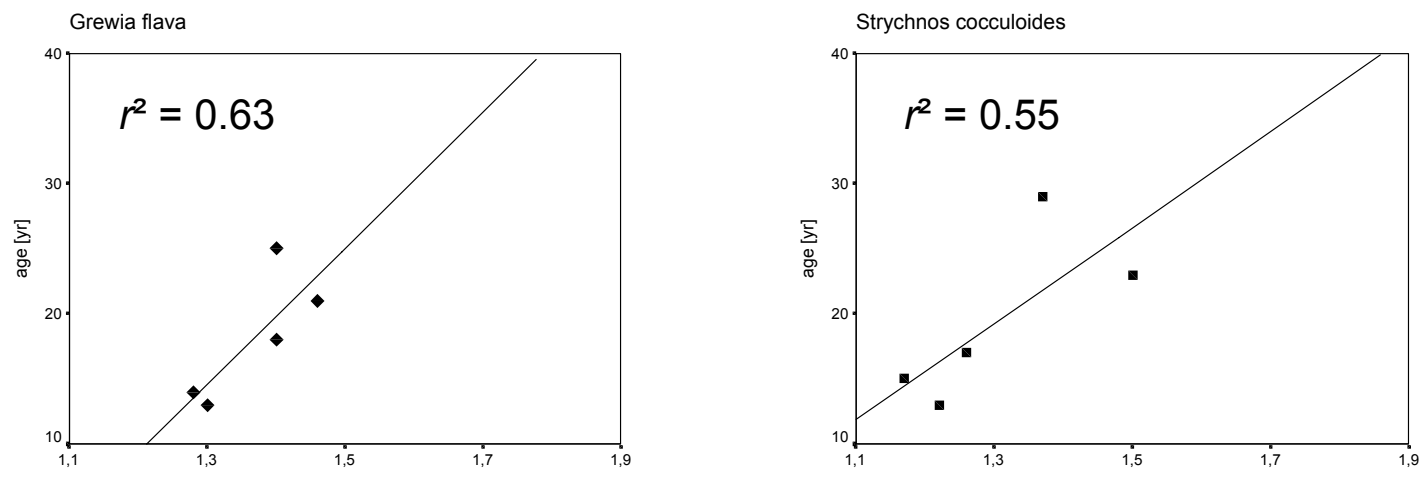

box counting dimension $\mathrm{D}$

box counting dimension $\mathrm{D}$
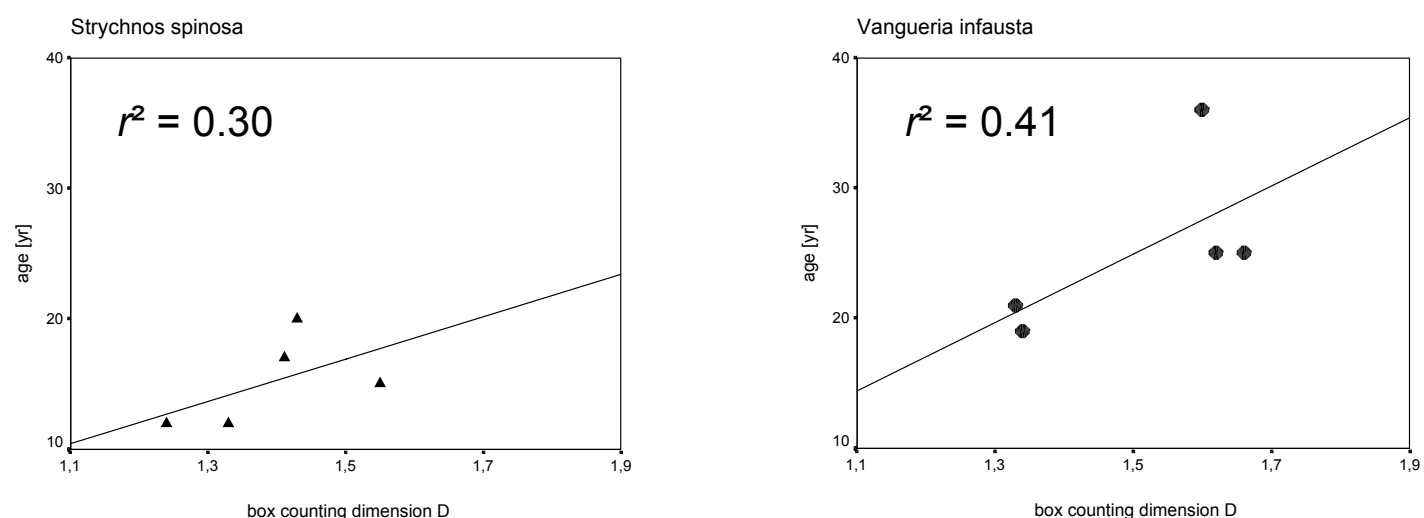

Figure 7. Age vs. box counting dimension $D$ from Grewia flava, Strychnos cocculoides, Strychnos spinosa and Vangueria infausta, with regression line for each species.

On the base of quantitative analysis with GROGRA 3.2, it was possible to test mathematical models at reconstructed virtual 3-D structures obtained from in situ measurements. Especially the fractal analysis seems to be a useful tool to quantify the exploration of a three dimensional space in a given range of scales, although the obtained box-counting dimensions have to be relativized in view of our artificial diameter threshold of $3 \mathrm{~mm}$. In a study of above-ground branching patterns of trees, where all segments down to the smallest diameter were measured (see [29] for 
details), we applied a fractal analysis with the same set of resolutions on a full system and on a system where all branches weaker than $3 \mathrm{~mm}$ were removed. The resulting dimension was diminished by 0.22 by the removal. We assume that the necessary correction of $D$ will be of the same order of magnitude in the case of our root systems, yielding a "true" $D$ approximately between 1.5 and 1.75. However, some uncertainty remains as the root branching patterns differ considerably from the above-ground patterns considered in the cited study.
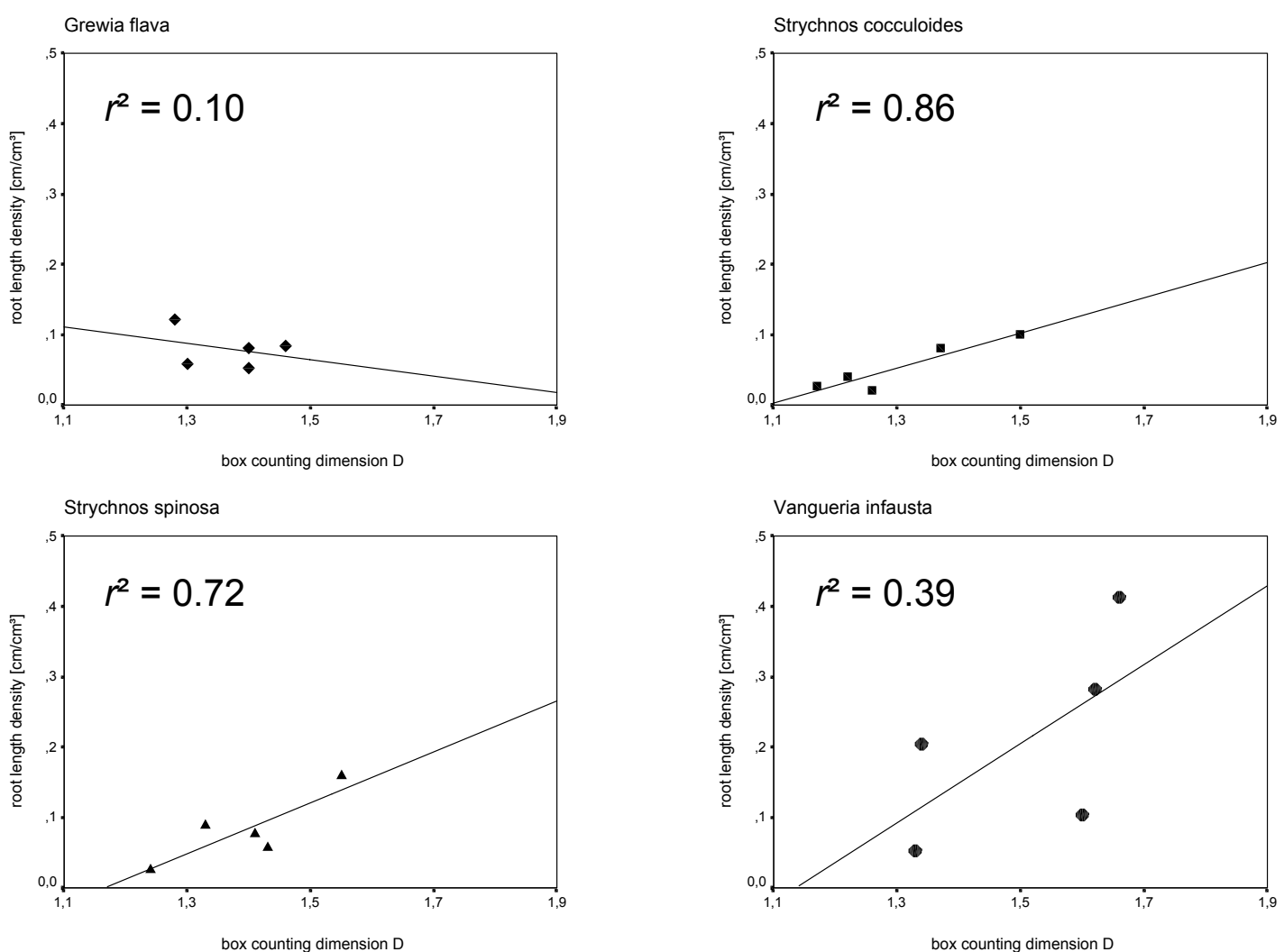

Figure 8. Root length density vs. box counting dimension $D$ from Grewia flava, Strychnos cocculoides, Strychnos spinosa and Vangueria infausta, with regression line for each species.

But, the comparison of different values of $D$ obtained with the same method and under the same restrictions can still indicate different degrees of exploration of the soil, with potential applications on agroforestry systems: The fractal dimension could be one possible indicator for competition between adjacent roots as well as for a more or less strong exploitation of soil resources.

The comparison of the $D$ values with the $D_{x y}$ values obtained from the projections of the root systems into the horizontal plane (Figure 9) shows that both dimensions are correlated, and that - over the range of scales considered, $-D_{x y}$ is not systematically smaller than $D$. However, $43 \%$ of the variation of $D$ cannot be explained by $D_{x y}$. Hence, a considerable loss of information occurs when only the (easier obtainable) 
dimension of the planar projection is calculated instead of carrying out the full 3-D analysis. However, our findings suggest that the results are not necessarily much worse when an even simpler method of soil exploration assessment is used to replace 3-D fractal analysis, i.e. the determination of overall root length density $\delta$ (cf. Figure 8). Here, $49 \%$ of the variation of $D$ remain unexplained, which is not much more than in the case when $D_{x y}$ is used to predict $D$. However, the apparent speciesdependence of the relationship between $\delta$ and $D$, and the small number of investigated individuals, make a further confirmation of these results desirable.

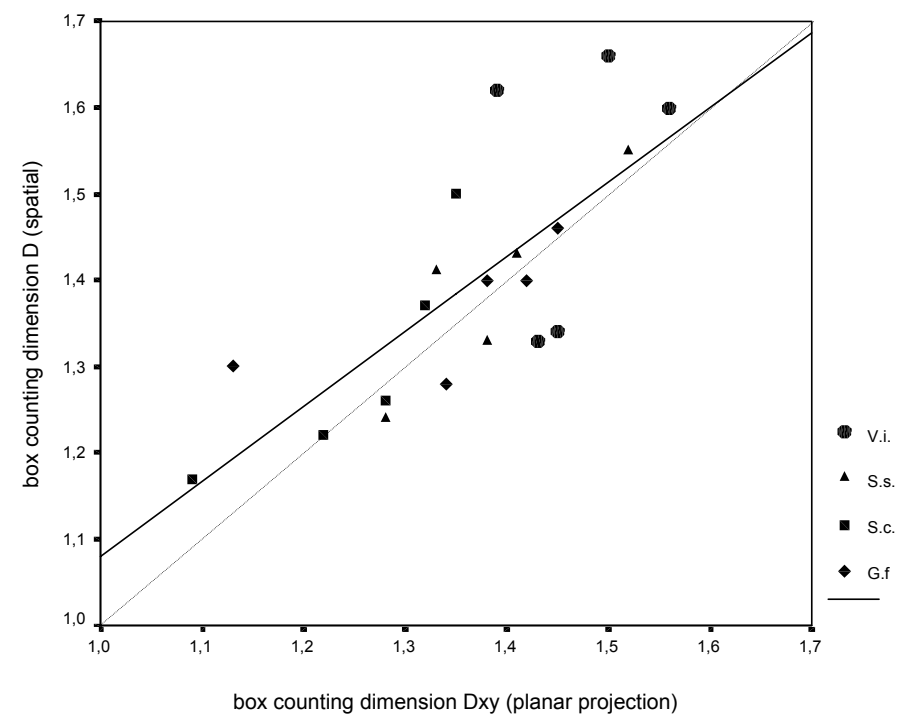

Figure 9. Correlation of box counting dimension $D$ (spatial) vs. $D_{x y}$ (planar projection) from Grewia flava (G.f.), Strychnos cocculoides (S.c.), Strychnos spinosa (S.s.) and Vangueria infausta (V.i.) , solid line: linear regression, dotted line: $D=D_{x y}$.

In the case of our fine root samples, we were restricted to a 2-D analysis method, i.e. we could only determine $D_{x y}$. The fine root samples do not show any significant differences in the box counting dimension between the species, whereas the coarse root systems do. A discrepancy in fractal dimension between coarse and fine root scale seems to occur at least for Grewia flava and Strychnos cocculoides, possibly due to a multifractal behaviour of the root systems, which is not unusual in natural phenomena [27, 34, 35].

The low variation of the average $D_{x y}$ for fine root samples $(1.41-1.48)$ between the species could be interpreted as an indicator of a species-independent rooting strategy. It seems that fine roots try to reach a certain value under almost similar environmental conditions which may be an optimum value under these growth conditions. Further comparison with similar analyses under different climatological and pedological conditions is needed for an interpretation of this almost constant value for $D_{x y}$.

Results from the analysis of the fractal dimension of the coarse root systems were consistent with the field observations. Strychnos cocculoides with the apparently 
weakest root system had the lowest box counting dimension $D$. The maximum value of $D$ for Vangueria infausta is in agreement with the branching orders and the branching intensity. These root systems are most intensively exploring the three dimensional space. However, the values of the box counting dimension for Stychnos spinosa and Grewia flava are surprising. From observations in the field it was expected that the latter had the more intensive coarse root system, whereas fractal analysis did not show a significant difference between these species. Surprising is that a higher branching order (maximum for Grewia flava: 3, Strychnos spinosa: 2) seems to be not necessarily a good indicator for the intensity of exploration of the soil.

When these results are considered, one has to keep in mind that the fractal or box counting dimension gives only a very condensed information about the spatial organization of the branching systems in the soil. To gain knowledge about factors influencing the activity of individual root meristems and about the ecophysiological strategies followed by the different species during the complete course of ontogenesis, dynamic studies including the observation of growing roots at different moments in time are probably necessary. We had to restrict ourselves to "static" descriptions. However, static observation also has advantages once the digital reconstruction is performed for a sample of root systems, characteristic features of the branching systems can be quickly detected and precisely quantified.

\section{Acknowledgements}

We are grateful to Mmoloki Botite and Golekwang Phiri for excellent assistance in the field, to Stefan Luther for support concerning the software tools, to Martin Worbes for dendrochronological assistance and to Ana M. Tarquis and Amram Eshel for helpful suggestions and literature. Furthermore, we express our thanks to two anonymous referees for their remarks which helped to improve the paper.

\section{References}

[1] Barnsley M.F., Fractals Everywhere, Associated Press, Boston, 1988.

[2] Berntson G.M., Fractal geometry, scaling, and the description of plant root architecture, in: Waisel Y., Eshel A., Kafkafi U. (Eds.), Plant Roots: The Hidden Half, Dekker, New York, 2nd ed., 1996, pp. 259-272.

[3] Colin-Belgrand M., Joannes H., Dreyer E., Pagès L., A new data processing system for root growth and ramification analysis: Description of methods, Ann. Sci. For. 46 Suppl. (1989) 305s-309s.

[4] Colin-Belgrand, Pagès L., Dreyer E., Joannes H., Analysis and simulation of the architecture of a growing root system: application to a comparative study of several tree seedlings, Ann. Sci. For. 46 Suppl. (1989) 288-293. 
[5] Coutts M.P., Development of the structural root system of Sitka spruce, Forestry 56 (1983) 1-16.

[6] Coutts M.P., Lewis G.J., When is the structural root system determined in Sitka spruce, Plant Soil 71 (1983) 155-160.

[7] De Reffye Ph., Houllier F., Modelling plant growth and architecture: Some recent advances and applications to agronomy and forestry, Current Sci. 73 (1997) 984992.

[8] De Reffye Ph., Fourcaud Th., Blaise F., Barthélémy D., Houllier, F., A functional model of tree growth and tree architecture, Silva Fenn. 31 (1997) 297-311.

[9] De Reffye Ph., Houllier F., Blaise F., Barthélémy D., Dauzat J., Auclair D., A model simulating above- and belowground tree architecture with agroforestry applications, Agrofor. Syst. 30 (1995) 175-197.

[10] De Wit P.V., Becker R.P., Explanatory note on the land system map of Botswana. FAO/UNDP/Government of Botswana. Soil Mapping and Advisory Services, Field Doc. 31, 1990.

[11] Deans J.D., Dynamics of coarse root production in a young plantation of Picea sitchensis, Forestry 54 (1981) 139-155.

[12] Danjon F., Sinoquet H., Godin C., Colin F., Drexhage M., Characterisation of the structural tree root architecture using digitising and the AMAPmod software, Plant Soil (submitted).

[13] Drexhage M., Gruber F., The architecture of woody root systems of 40-year-old Norway spruce (Picea abies (L.) Karst.): crown-trunk-relations and branching forms, in: Kutschera L. (Ed.), Root Ecology and its Practical Application, Klagenfurt, 1992, pp. 703-706.

[14] Edgar G., Measures, Topology and Fractal Geometry, Springer, New York, 1990.

[15] Eshel A., On the fractal dimension of a root system, Plant, Cell Env. 21 (1998) 247-251.

[16] Falconer K., Fractal Geometry: Mathematical Foundations and Applications, Wiley, New York, 1990.

[17] Fayle D.C.F., Extension and longitudinal growth during the development of Red Pine root systems, Can. J. For. Res. 5 (1975) 109.

[18] Fitter A.H., Stickland T.R., Harvey M.L., Wilson G.W., Architectural analysis of plant root systems. 1. Architectural correlations of exploitation efficiency, New Phytol. 118 (1991) 375-382. 
[19] Godin C., Guédon Y., Costes E., Caraglio Y., Measuring and analysing plants with the AMAPmod software, in: Michalewicz M.T. (Ed.), Advances in Computational Life Sciences, Vol. I: Plants to Ecosystems, CSIRO, Brisbane, 1997, pp. 53-84.

[20] Gruber R., Dynamik und Regeneration der Gehölze, Berichte des Forschungszentrums Waldökosysteme, Ser. A, Vol. 86, Göttingen, 1992.

[21] Hall P., Wood A., On the performance of box-counting estimators of fractal dimension, Biometrika 80 (1993) 246-252.

[22] Hallé F., Oldeman R.A.A., Tomlinson P.B., Tropical Trees and Forests, Springer, Berlin, 1978.

[23] Henderson R., Ford E.D., Rensaw R., Deans, J.D., Morphology of the structural root system of Sitka spruce 1. Analysis and quantitative description, Forestry 56 (1983) 122- 135.

[24] Jourdan C., Rey H., Guédon Y., Architectural analysis and modelling of the branching process of the young oil-palm root system, Plant Soil 177 (1995) 63-72.

[25] Jourdan C., Rey H., Modelling and simulation of the architecture and development of the oil-palm (Elaeis guineensis Jacq.) root system I. The model, Plant Soil 190 (1997) 217-233.

[26] Jourdan C., Rey H., Architecture and development of the oil-palm (Elaeis guineensis Jacq.) root system, Plant Soil 189 (1997) 33-48.

[27] Kaye B.H., A Random Walk Through Fractal Dimensions, VCH, Weinheim, 1989.

[28] Kurth W., Growth Grammar Interpreter GROGRA 2.4, Berichte des Forschungszentrums Waldökosysteme, Ser. B, Vol. 38, Göttingen, 1994.

[29] Kurth W., Die Simulation der Baumarchitektur mit Wachstumsgrammatiken, Habilitationsschrift, Georg-August-Universität Göttingen, 1998.

[30] Kurth W., Anzola Jürgenson G., Triebwachstum und Verzweigung junger Fichten in Abhängigkeit von den beiden Einflußgrößen "Beschattung" und "Wuchsdichte": Datenaufbereitung und -analyse mit GROGRA, in: Pelz D. (Ed.), DVFF Sektion Forstliche Biometrie und Informatik, 10. Tagung Freiburg i. Br. 24.-26. 9. 1997, Biotechn. Fakultät, Ljubljana, 1997, pp. 89-108.

[31] Lungley D.R., The growth of root systems - A numerical computer simulation model, Plant Soil 38 (1973) 145-159.

[32] Lyford W.H., Development of the Root System of Northern Red Oak (Quercus rubra L.), Harvard Forest Paper 21 (1980) 3-30. 
[33] Mandelbrot B.B., The Fractal Geometry of Nature, W.H. Freeman, New York, 1982.

[34] Mandelbrot B.B., Multifractal measures, especially for the geophysicist, Pure Appl. Geophys. 131 (1989) 5-42.

[35] Mark D.M., Aronson P.B., Scale-dependent fractal dimensions of topographic surfaces: An empirical investigation, with applications in geomorphology and computer mapping, Math. Geol. 16 (1984) 671-683.

[36] Pagès L., Kervella J., Growth and development of root systems: Geometrical and structural aspects, Acta Biotheor. 38 (1990) 289-302.

[37] Reeve R., A warning about standard errors when estimating the fractal dimension, Comp. Geosci. 18 (1992) 89-91.

[38] Room P.M., Maillette L., Hanan J.S., Module and metamer dynamics and virtual plants, Adv. Ecol. Res. 25 (1994) 105-157.

[39] Sinoquet H., Rivet P., Measurement and visualization of the architecture of an adult tree based on a three-dimensional digitising device, Trees 11 (1997) 265-270.

[40] Stoll P., Modular growth and foraging strategies in rhizome systems of Solidago altissima L. and branches of Pinus sylvestris L., Dissertation, Universität Zürich, Philosophische Fakultät II, 1995.

[41] Strand L., Crown density and fractal dimension, Meddelelser fra Norsk Institutt for Skogforskning 43 (1990) 1-11.

[42] Tatsumi J., Yamauchi A., Kono Y., Fractal analysis of plant root systems, Ann. Bot. 64 (1989) 499-503.

[43] Theiler J., Statistical precision of dimension estimators, Phys. Rev. A 41 (1990) 3038-3051.

[44] Zeide B., Gresham C.A., Fractal dimensions of tree crowns in three Loblolly pine plantations of coastal South Carolina, Can. J. For. Res. 21 (1991) 1208-1212.

[45] Zeide B., Pfeifer P., A method for estimation of fractal dimension of tree crowns, For. Sci. 37 (1991) 1253-1265. 
The following article

Topology, scaling relations and Leonardo's rule in root systems from African tree species

contributed by:
A.L Oppelt,
W. Kurth,
D.L. Godbold

was published in:

Tree Physiology 21 (2001)

on pages:

$117-128$ 



\section{Topology, scaling relations and Leonardo's rule in root systems from African tree species}

\section{Summary}

Aspects of root architecture, including topology, link length, diameter and scaling relations, were analysed in excavated coarse root systems of three field-grown fruit tree species (Strychnos cocculoides Bak., Strychnos spinosa Lam. and Vangueria infausta Burch.) and the fruit-bearing shrub Grewia flava DC. We investigated the root systems using semi-automatic digitizing and computer-based 3-D reconstruction techniques. Topological analysis was carried out to investigate branching patterns as basic determinants of root architecture. New topological indices were developed and revealed significant differences among the species. The different architectural strategies can be explained in terms of cost-benefit relations and efficiency in soil resource exploration and exploitation. In addition, some well-known hypotheses about geometry and scaling, most of them previously unverified by empirical observations on root systems, were tested. For practical applications, the main emphasis is on the relationship between proximal root diameter, an easily determined parameter, and several parameters describing the size of the whole root system. We also tested the "pipe stem" theory, essentially dating back to Leonardo da Vinci, which underlies many models and which we found conformed to our measurement data with reasonable accuracy. A physiological consequence of the "constant cross-sectional area rule" may be a certain homogeneity of hydraulic architecture throughout root systems.

\section{Introduction}

Investigation of tree roots is laborious and time consuming. Root systems of trees have, therefore, been much less frequently studied than aerial parts. In this paper, information about topology and geometrical scaling in coarse roots of fruit tree species native to Southern Africa are presented. Root systems of the fruit trees Strychnos cocculoides Bak., Strychnos spinosa Lam. and Vangueria infausta Burch., as well as one shrubby species, Grewia flava DC., were investigated. Beside gathering information about these rarely investigated plants, we wanted to develop new techniques of root structural reconstruction and analysis. We also evaluated some well-known hypotheses about topology and scaling that might be of general interest in root system research, but are seldom tested empirically. We place this study in the context of functional and structural modeling of plants (Sievänen et al. 1997, Cruiziat 1998), topological analysis (Fitter et al. 1991, Berntson 1997) and the 
assessment of strategies for soil exploration and exploitation (Gandar and Hughes 1988, Hughes et al. 1995).

\section{Topology}

Topological properties of root systems have received considerable attention in the literature (Fitter 1985, 1986, 1987, Fitter and Stickland 1991, Spek and van Noordwijk 1994, Bert et al. 1998). They are believed to influence the efficiency and costs of resource exploitation in terms of carbon required for root segment construction (Fitter 1986, Fitter et al. 1991). One of the main goals of topological analysis is to find out whether habitat conditions lead to evolutionary adaptations in branching behavior and rooting strategy.

Various theoretical approaches to evaluating topology have been developed (Fitter 1985, 1986, van Noordwijk et al. 1994, Berntson 1997) and have been compared on theoretical grounds (Berntson 1995), but practical applications have, in most cases, been restricted to herbaceous plants (Fitter 1986, Fitter and Stickland 1991). Furthermore, some of the topological indices developed to compare root systems of different sizes show either an unstable oscillating behavior in random simulations (Berntson 1995) or are based on randomness assumptions that were originally developed for planar networks (see Werner and Smart 1973) and are inadequate for 3-D branching systems (Fitter 1986).

One of the most often cited topological distinctions is that between "dichotomous" and "herringbone" branching patterns (Fitter 1986, 1987, Fitter et al. 1991, van Noordwijk et al. 1994, Lynch 1995, Berntson 1997). We introduce two new indices to quantify branching patterns in the continuum between these extremes, and apply them to reveal species-specific characteristics of the root systems we investigated. This approach is complemented by the analysis of exterior and interior link lengths. These are metric values, but they are closely related to topological structure. The graph-theoretical background to our topological analysis is outlined in the Theory section below.

\section{Diameter and branching}

When examining metric properties of root systems, the question arises how the diameters before and after branching nodes are related to each other. Leonardo da Vinci claimed in his notebook that the cross-sectional area of a trunk or branch of a tree is equal to the sum of the cross-sectional areas of the branches at any higher level (Richter 1970). Locally, this means that in each branching node where $n$ daughter branches emerge, the diameter $d$ before the node is related to the diameters $d_{i}(i=1, \ldots, n)$ (Figure 1$)$ of the daughter segments by: 


$$
d^{2}=\sum_{i=1}^{n} d_{i}^{2}
$$

For a system of conducting tubes with negligible flow resistance, this condition enables equal flow rates throughout the system. Equation 1 was used in a large number of models of plant functioning and growth (e.g., Shinozaki et al. 1964, Perttunen et al. 1996) and was popularized under the names "pipe stem theory" (John Ruskin, see MacDonald 1983) or "pipe model." Examples of theoretical considerations building on Equation 1 are Mendès France (1981) and Long (1994).

Various generalizations of Equation 1 have been considered. One of them is:

$$
d^{\Delta}=\sum_{i=1}^{n} d_{i}^{\Delta}
$$

where the paramete $\Delta$ is called the diameter exponent of the system (Mandelbrot 1983). Murray (1927) observed that in a number of aboveground tree branching systems that have been investigated, weight was approximately proportional to $d^{2.5}$, where $d$ is the proximal diameter. From this he concluded (using additivity of weight in the branching nodes) that $=2.5$ should hold constant. However, his argument was too simple, because weight is additive only if the contribution of the mother segment of the branches is neglected. Recalculating $\Delta$, Mandelbrot (1978) obtained a value of 2 , thus confirming Leonardo's rule.

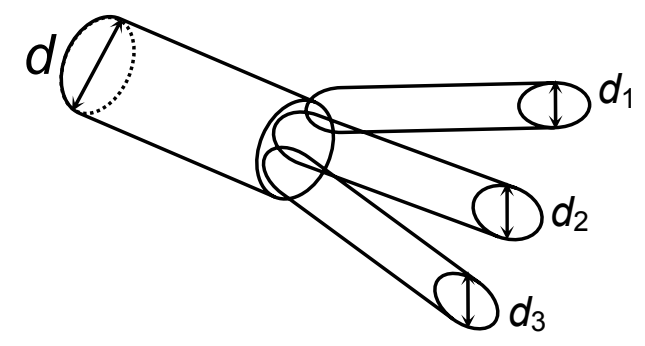

Figure 1. Principle of Leonardo's rule, which is expressed mathematically in Equation 1.

Surprisingly, direct empirical estimations of $\Delta$ are rare. Mandelbrot (1983) mentioned a reexamination of data obtained by McMahon and Kronauer (1976) from three tree crowns, yielding a $\Delta$ near 2 or slightly below. An examination of three young Picea abies (L.) KARST. crowns (Anzola Jürgenson 1998) showed no significant deviation from $\Delta=2$. We are unaware of corresponding investigations of root systems.

Another generalization of Equation 1, utilized by van Noordwijk et al. (1994) and Spek and van Noordwijk (1994) in theoretical studies, is:

$$
d^{2}=\alpha \sum_{i=1}^{n} d_{i}^{2}
$$


with a proportionality factor $\alpha>0$. We checked Equations 2 and 3 with our root system data.

\section{Scaling relations}

There is much in the literature about scaling relationships and allometries of whole plants or plant organs; see, e.g., Niklas (1994) for a general overview. We were motivated to investigate the tapering of our roots by the work of McMahon and Kronauer (1976) on branch tapering in tree crowns. They related the diameter $d$ of a segment, taken at some point in the branching system, to the average length $L_{p}$ of all paths going from that segment distally to a branch tip.

From the theory of elastic similarity, they deduced a relationship of the form $d=\gamma\left(L_{p}+I_{0}\right)^{\beta}$, with constants $\gamma, I_{0}$ and $\beta$ and with $\beta=1.5$, which was confirmed by their empirical data. Because the mechanical argument does not apply to root systems, the question arose whether the same relationship with a possibly different value of $\beta$ (or a different form of regression equation between $d$ and $L_{p}$ ) holds for roots.

Another relationship, perhaps of greater practical significance, is that between the diameter $d$ of a segment and the total length sum $L$ of all roots distal to that segment. A tight correlation between these values, applied to the diameter at the root collar or to the diameters of the main roots near the collar, could be useful in estimating total root length. On the basis of self-similarity assumptions and Equation 3, van Noordwijk et al. (1994) and Spek and van Noordwijk (1994) obtained a proportionality between $L$ and $d^{2}$ in artificially constructed branching patterns. However, they did not seek empirical confirmation of this relationship in the field.

Recently, West et al. (1997) derived scaling relations for vessel systems from the principle of minimal hydraulic resistance and from self-similarity assumptions. Their model coincided with empirical evidence from cardiovascular and respiratory systems of animals, and they claimed to have found "a general model for the origin of allometric scaling laws in biology." Applied to the mass $m$ of the organism or organ to be fed and to the proximal diameter $d$ of the supplying vessel, their model predicts the relation $d=\lambda m^{\varepsilon}$, where $\lambda$ and $\varepsilon$ are constants and $\varepsilon=3 / 8$. We checked this relationship on the root systems by inserting the sum of the volumes of all root segments for $m$, assuming that this total volume is proportional to $m$, and root collar diameter for $d$.

\section{Theory}

\section{Graphs of branching patterns}

Topology studies the adjacency properties of objects, ignoring their metric sizes (e.g., lengths, angles, diameters). From the topological viewpoint, a root system is a graph 
in the sense of graph theory (e.g., Deo 1974), i.e., an object consisting of vertices and edges (links), each connecting two vertices. A graph is completely described by the information specifying which vertices are connected by an edge and which are not. Because we have found no anastomosis in our root systems, we can further restrict the class of graphs to so-called "trees", that is, graphs without cycles. The root collar, where the aboveground part of the plant begins, corresponds to a special vertex of the underlying graph, known in mathematical terminology as the root of the tree. However, to avoid confusion, we refer to the mathematical root, which in our application is the root collar or the shoot, as the base vertex. In our topological drawings the base vertex will be represented by an empty circle. The base vertex can serve to assign a unique direction to every link giving rise to a so-called directed tree. In our analyses, we assume a distal orientation of the system, biologically corresponding to the flow of assimilates from the shoot to the root tips (Figure 2 (the arrows are omitted in subsequent figures)).

Each link not emerging from the base vertex has indegree 1 (i.e., it is adjacent to one mother link in the proximal direction), and links can have various outdegrees, indicating the number of adjacent daughter links in the distal direction (Figure 2). We denote by $v_{\mathrm{k}}$ the number of links of outdegree $k(k=0,1,2 \ldots)$. When we omit the base vertex, we have an equal number $v=v_{0}+v_{1}+v_{2}+\ldots$ of vertices and links.

In the literature, root systems are usually represented by binary trees, i.e., by trees where every link has outdegree 2 or 0 , and every vertex corresponds to a dichotomous branching node or to a root tip. However, in our field observations we found situations where two successive root segments had very different morphological characteristics, e.g., when an old axis had stopped apical growth but was the mother segment of a single, much younger reiterative root. In these cases we assigned a higher botanical order to the reiterative root, and we did not merge segments of different order to a single link. As a consequence, we obtained some links of outdegree 1 in our topological graphs. Furthermore, there were cases where several branches emerged from the same mother segment at nearly identical positions (distance of branching nodes $<5 \mathrm{~mm}$ ), and we refrained from inserting artificial "minilinks" only to maintain the binary branching law. Hence we have some links of outdegree 3 or higher. This leads to a loss of mathematical elegance for the sake of realism. However, the number of these "exceptional" links remained limited, and binary branching can still be considered the normal case.

In binary trees, the number $v_{0}$ of exterior links (often referred to as the magnitude of the tree, e.g., Fitter 1986) determines the number $v$ of all links:

$$
v=2 v_{0}-1
$$


(e.g., Deo 1974, Tucker 1980). In our general case, we must modify Equation 4 by a correction term or discrepancy $(\delta)$ :

$$
v=2 v_{0}-1+\delta,
$$

with

$$
\delta=\sum_{k=1}^{\infty}(2-k) v_{k}
$$

Only "exceptional" links contribute to $\delta$ (notice that $v_{2}$ makes no contribution in Equation 6), and, in contrast to $v_{1}$, all $v_{k}$ with $k>2$ have a negative coefficient.

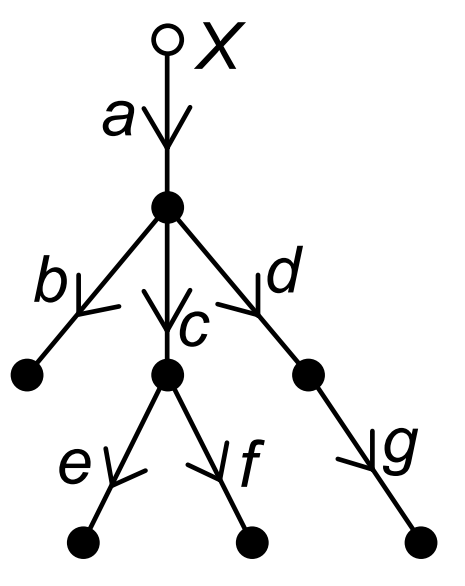

Figure 2. Example of a directed graph representing a root system topologically. Terminology: $X$ is the base vertex, $b, e$, $\mathrm{f}, \mathrm{g}$ are exterior links, $\mathrm{a}, \mathrm{c}, \mathrm{d}$ interior links, where a has outdegree 3 , c outdegree 2 , and $d$ outdegree 1 . The topological depth of $b$ is 2 (measured always from $X$ ), that of $\mathrm{e}, \mathrm{f}$ and $\mathrm{g}$ is 3 .

\section{Topological indices}

The most obvious topologically extreme branching forms in the binary case occur, on the one hand, for the complete dichotomous pattern with $2^{n}$ exterior links of equal distance to the base, and on the other hand, for a single axis with only one exterior link emerging at each vertex (herringbone pattern; Fitter 1986, 1987, van Noordwijk et al. 1994). To quantify the position of an arbitrary binary tree between these extremes, it is necessary to define a topological equivalent of "rooting depth". The topological depth of an exterior link is the number of links in the unique directed path from the base vertex of the graph to the end vertex of the link in question (see Figure 2; terminology in accordance with the notion of "depth-first search" in computer science, e.g., Grimaldi 1989). The maximal topological depth or altitude (a) of a directed tree is the number of links in the (topologically) longest directed path. This parameter, which is in the binary case minimal for the complete dichotomous and maximal for the herringbone pattern, is named diameter (Fitter 1986) or altitude (Fitter 1987, Fitter et al. 1991, Berntson 1995) in the literature on root research and height in most textbooks on graph theory or discrete mathematics (e.g., Deo 1974, Liu 1977, Tucker 1980, Grimaldi 1989). Somewhat reluctantly, we continue to use the term altitude here. As a second parameter, we calculate the mean topological depth 
$b$ of a directed tree as the average topological depth of all exterior links. Parameter $b$ is of the same order of magnitude as $a$, and it is related to $p_{e}$, the sum of all pathlengths from the base to the exterior links, which is often used in the literature (exterior pathlength: Fitter 1986, total exterior pathlength: Fitter and Stickland 1991, total external path length: Berntson 1995, path length: Deo 1974), by:

$$
b=p_{e} / v_{0}
$$

The range of possible values of $a$ and $b$ grows with the magnitude $v_{0}$ of the root system. To enable comparisons of branching patterns of different sizes, Fitter (1985) introduced indices based on expected values of $a$ and $p_{\mathrm{e}}$ under a specific random model (Werner and Smart 1973). However, this model was derived for networks in geography and only makes sense for planar patterns. Unfortunately, the precise expected values and confidence intervals calculated by Berntson (1995) are also based on this planarity assumption and are therefore inappropriate for 3-D root branching patterns. We decided to avoid randomness assumptions; instead, we simply normed both parameters $a$ and $b$ by a linear transformation, making their minimal and maximal values for binary trees (given by Knuth 1973, Fitter 1986) 0 and 1 , respectively. The explicit definitions of both resulting normed indices $q_{a}$ and $q_{b}$, that are confined to the interval $[0 ; 1]$ for binary trees (but can take values beyond these limits if $v_{1}>0$ or $v_{3}>0$ ), are:

$$
q_{a}=\frac{a-1-l b v_{0}}{v_{0}-1-l b v_{0}}, \quad q_{b}=\frac{b-1-l b v_{0}}{\left(v_{0}+1\right) / 2-v_{0}^{-1}-l b v_{0}}
$$

where $\mathrm{Ib} v_{0}\left(=\ln v_{0} / \ln 2\right)$ is the binary logarithm.

The behavior of these indices is demonstrated in Figure 3 for the six possible binary trees for which one link emerges from the base. The pattern to the lower right is the herringbone type, corresponding to $q_{a}=q_{b}=1$, whereas the value $q_{a}=q_{b}=0$ would occur only for a perfectly dichotomous pattern.

The value of $q_{b}$ follows a more continuous course between the extremes compared with $q_{a}$. Furthermore, Berntson (1995) identified the related parameter $p_{e}$ in a series of growth simulations using the Monte-Carlo technique as that with the least erratic and most size-independent and stable behavior among several other topological indices. We therefore advocate the use of $q_{b}$, but in our empirical study we have also calculated a (and $q_{a}$ ) because of its simple definition.

Two further parameters related to topological structure are the mean lengths of exterior and interior links. Fitter (1986, 1987) and Fitter and Stickland (1991) intensively investigated these link lengths and their relation to water and nutrient supply. 


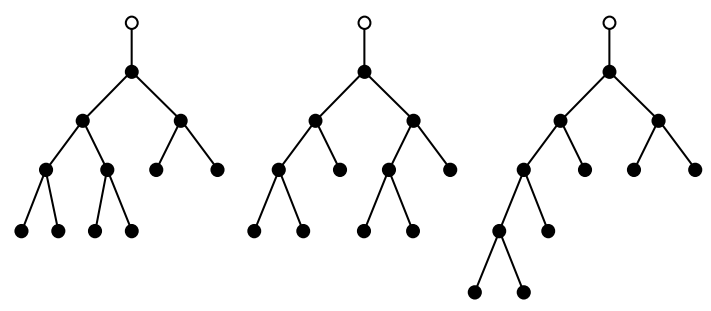

\begin{tabular}{llll}
$a$ & 4 & 4 & 5 \\
\hline$q_{a}$ & 0.172 & 0.172 & 0.586 \\
\hline$b$ & 3.667 & 3.667 & 3.833 \\
\hline$q_{b}$ & 0.109 & 0.109 & 0.332 \\
\hline
\end{tabular}
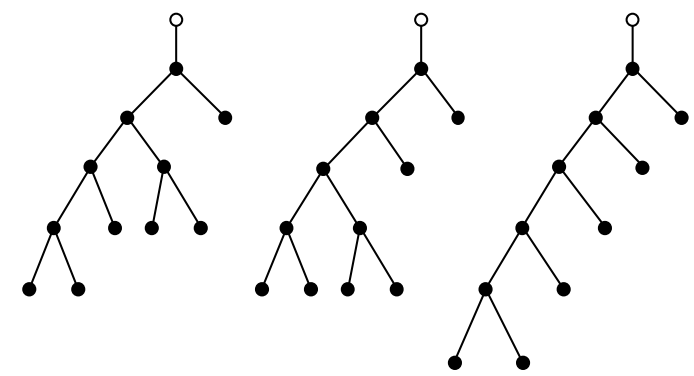

\begin{tabular}{llll}
$a$ & 5 & 5 & 6 \\
\hline$q_{a}$ & 0.586 & 0.586 & 1.0 \\
\hline$b$ & 4.0 & 4.167 & 4.333 \\
\hline$q_{b}$ & 0.555 & 0.777 & 1.0
\end{tabular}

Figure 3. The six topologically distinct binary trees with $v_{0}=6$ distal edges (links), together with their respective altitude $a$, mean topological depth $b$ and with the corresponding normed indices $q_{a}$ and $q_{b}$. They represent the range from dichotomous to herringbone branching. The parameter $b$ (or $q_{b}$ ) allows a better distinction between the types than parameter $a$, though both $a$ and $b$ fail to distinguish between the two upper leftmost types. Notice that the theoretical minimum $q_{a}=q_{b}=0$ is not reached in this case because 6 is not of the form $2^{n}$.

\section{Materials and Methods}

The study site is located on sandveld near Mogorosi (Serowe Region, Central District, Botswana) between longitude $26^{\circ} 36.26^{\prime}$ and $26^{\circ} 36.70^{\prime} \mathrm{E}$ and latitude from $22^{\circ} 25.09^{\prime}$ to $22^{\circ} 25.30^{\prime} \mathrm{S}$. For a more detailed site description see Oppelt et al. (2000).

The architecture of in situ-grown coarse root systems of the fruit tree species Strychnos cocculoides (Loganiaceae), Strychnos spinosa and Vangueria infausta (Rubiaceae), as well as from the shrub Grewia flava (Tiliaceae) was studied. Coarse roots were defined as roots that exceed a threshold diameter of $3 \mathrm{~mm}$. A reconstruction of spatial orientation and branching below that value was not possible.

Each species was represented by five coarse root systems, which were excavated by hand. Each exposed root was divided into segments of variable length, according to changes in growth direction or at positions where daughter roots were emerging, and marked with white ink. The spatial orientation (vertical and horizontal angle) as well as the length of each segment in its original position was determined with a digital compass (TECTRONIC 4000, Breithaupt, Kassel, Germany) and automatically recorded (L-file).

After spatial measurements, coarse roots were removed, the diameter of each segment measured with a digital caliper (PM 200, HHW Hommel, Switzerland), and the data recorded in a D-file corresponding to each L-file. 


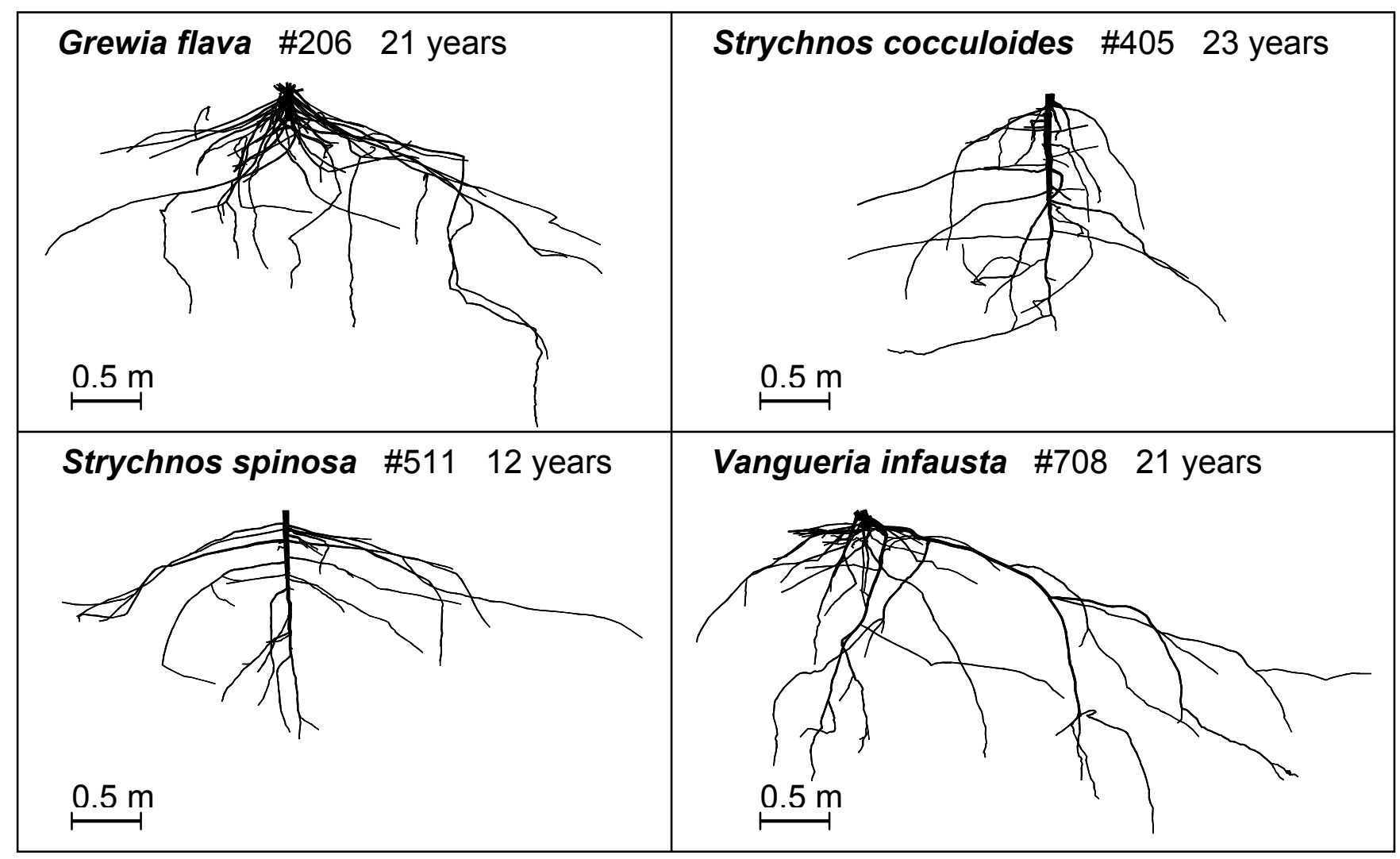

Figure 4. Lateral view of four reconstructed root systems (graphical output from GROGRA). The age of each tree is also indicated.

Both raw data sets ( $L$ - and D-files) were merged by self-authored interface software, creating the final code for reconstruction. As a basis for the topological description of the branching systems, a developmental botanical concept of branching order was applied: the order of the tap root (if it exists) is 0 , and an $n$th order root has branches of order $n+1$. The branching order was calculated for each segment automatically. For encoding the full geometrical and topological structure of the root systems (lengths, orientations and diameters of all segments and mother-segment linkages) we used the digital tree data format (dtd code, Kurth 1994). The dtd files, each representing a complete root system, were generated semi-automatically as described above.

The software GROGRA 3.2 (Kurth 1994) was used to reconstruct the architecture of individual root systems from the data files in the form of a linked list data structure. Lateral views of one example root system of each species, obtained from the software as graphical output, are shown in Figure 4.

The GROGRA software can extract different kinds of graphical and numerical information from the virtual 3-D structures. Metric information about each root segment and about the whole system was written into tabular files and processed with the SPSS data analysis software (SPSS 8.0, SPSS Inc., Chicago, IL) and Statistica v. 5 (StatSoft Inc., Tulsa, OK). Topological analysis was enabled using a 
transformation function in GROGRA, which was originally devised as an interface for a numerical water-flow simulator for tree crowns (Früh and Kurth 1999). It unifies each chain of subsequent unbranched root segments of the same botanical order into one link. Afterwards, the lengths of all links were artificially reduced to one to enable topological depth calculations with the same algorithms that had previously yielded the metric pathlengths.

\section{Results}

Coarse root systems from Grewia flava were characterized by an intensive shallow network of slowly tapering first-order laterals. Structural roots with higher branching orders developed mostly in a vertical direction, and exploited deeper soil layers. Functionally, these replaced the frequently absent taproot. An intensive development of adventitious roots, especially on older individials, was observed.

In contrast to the other root systems investigated, both Strychnos species were characterized by a deep and prominent taproot, as a result of secondary growth. Branching intensity was low and branching orders did not normally exceed 2 , so that they can be described as weakly exploiting root systems. Both species are distinguished by their vertical root distribution. Strychnos cocculoides showed the maximum amount and horizontal extent of first-order laterals in deeper subsoil layers, whereas Strychnos spinosa showed a greater horizontal extension of lateral roots with a high concentration in the topsoil.

In contrast, root systems of Vangueria infausta showed higher branching intensity. First-order laterals were distinctly shorter and branched rapidly into higher orders. Most laterals initiated in the horizontal plane, but changed with time to a more vertical orientation. If present, taproots tapered rapidly, never reaching great depth (Figure 4, see also Oppelt et al. 2000).

Quantitative characteristics of all the root systems investigated are given in Tables 1 and 2 .

\section{Topological indices}

Figure 5 shows the results of the calculation of the topological indices $q_{a}$ and $q_{b}$ (numerical values given in Table 1), grouped according to species. 
Table 1. Topological parameters of the root systems of each sample tree.

\begin{tabular}{|c|c|c|c|c|c|c|c|c|c|}
\hline species & tree & age & $\left.\boldsymbol{v}^{1}\right)$ & $\left.v_{0}{ }^{2}\right)$ & $\left.\delta^{3}\right)$ & $\left.a^{4}\right)$ & $\left.b^{5}\right)$ & $\left.q_{a}{ }^{6}\right)$ & $\left.q_{b}{ }^{6}\right)$ \\
\hline \multirow{5}{*}{$\begin{array}{l}\text { Grewia } \\
\text { flava }\end{array}$} & 203 & 25 & 252 & 138 & -23 & 23 & 9.41 & 0.11 & 0.02 \\
\hline & 206 & 21 & 127 & 78 & -28 & 10 & 4.09 & 0.04 & -0.1 \\
\hline & 213 & 18 & 100 & 54 & -7 & 16 & 8.09 & 0.2 & 0.06 \\
\hline & 214 & 14 & 47 & 23 & 2 & 11 & 4.74 & 0.31 & -0.11 \\
\hline & 215 & 13 & 37 & 20 & -2 & 13 & 6.45 & 0.52 & 0.18 \\
\hline mean & & 18.2 & 112.6 & 63 & -12.4 & 14.6 & 6.56 & 0.24 & 0.01 \\
\hline$s d$ & & 4.97 & 86.34 & 49.2 & 14.15 & 5.22 & 2.23 & 0.19 & 0.12 \\
\hline \multirow{5}{*}{$\begin{array}{l}\text { Strychnos } \\
\text { cocculoides }\end{array}$} & 405 & 23 & 92 & 50 & -7 & 31 & 13.6 & 0.58 & 0.36 \\
\hline & 409 & 15 & 39 & 20 & 0 & 16 & 9.9 & 0.73 & 0.75 \\
\hline & 412 & 17 & 25 & 13 & 0 & 12 & 7.62 & 0.88 & 0.9 \\
\hline & 425 & 29 & 76 & 38 & 1 & 23 & 12.6 & 0.53 & 0.45 \\
\hline & 426 & 13 & 28 & 14 & 1 & 12 & 7.79 & 0.78 & 0.82 \\
\hline mean & & 19.4 & 52 & 26.8 & -0.6 & 18.8 & 10.3 & 0.7 & 0.66 \\
\hline$s d$ & & 6.54 & 30.21 & 15.96 & 2.51 & 8.17 & 2.73 & 0.14 & 0.24 \\
\hline \multirow{5}{*}{$\begin{array}{l}\text { Strychnos } \\
\text { spinosa }\end{array}$} & 501 & 20 & 127 & 73 & -18 & 33 & 18.2 & 0.39 & 0.36 \\
\hline & 508 & 12 & 48 & 26 & -3 & 15 & 8.08 & 0.46 & 0.27 \\
\hline & 509 & 17 & 16 & 9 & -1 & 5 & 3.89 & 0.17 & -0.16 \\
\hline & 510 & 15 & 86 & 48 & -9 & 27 & 13.1 & 0.49 & 0.34 \\
\hline & 511 & 12 & 67 & 35 & -2 & 22 & 11.8 & 0.55 & 0.44 \\
\hline mean & & 15.2 & 68.8 & 38.2 & -6.6 & 20.4 & 11 & 0.41 & 0.25 \\
\hline$s d$ & & 3.42 & 41.55 & 24.08 & 7.09 & 10.85 & 5.38 & 0.15 & 0.24 \\
\hline \multirow{5}{*}{$\begin{array}{l}\text { Vangueria } \\
\text { infausta }\end{array}$} & 706 & 36 & 371 & 188 & -4 & 18 & 11 & 0.05 & 0.03 \\
\hline & 708 & 21 & 114 & 61 & -7 & 16 & 9.36 & 0.17 & 0.1 \\
\hline & 711 & 19 & 75 & 35 & 6 & 16 & 8.63 & 0.34 & 0.19 \\
\hline & 712 & 25 & 92 & 48 & -3 & 19 & 10 & 0.3 & 0.18 \\
\hline & 713 & 25 & 123 & 60 & 4 & 17 & 10.8 & 0.19 & 0.16 \\
\hline mean & & 25.2 & 155 & 79.4 & -2.8 & 17.2 & 9.96 & 0.21 & 0.13 \\
\hline$s d$ & & 6.57 & 122.2 & 64.38 & 8.17 & 1.3 & 0.99 & 0.11 & 0.07 \\
\hline $\begin{array}{l}{ }^{1} v=\text { Number } \\
{ }^{2} v_{0}=\text { Numbe } \\
{ }^{3} \delta=\text { Discrep } \\
{ }^{4} a=\text { Altitude } \\
{ }^{5} b=\text { Mean to } \\
6\end{array}$ & $\begin{array}{l}\text { mal } \\
\text { cal }\end{array}$ & pth & n) & & & & & & \\
\hline
\end{tabular}

Both indices are closely correlated with each other (Pearson's $r=0.92)$. A onefactorial ANOVA with species as factor showed a highly significant effect on $q_{a}(F=$ 11.2, $p=0.00033)$ and $q_{b}(F=11.7, p=0.00026)$. This effect was even more pronounced when the age of the tree was considered as a covariate $\left(q_{a}: F=20.5, p\right.$ $\left.=1.4 \times 10-5 ; q_{b}: F=14.7, p=9.8 \times 10-5\right)$. The root system of Tree 509, a Strychnos spinosa specimen with clear reiterative growth because of damage and an unusual growth habit, had atypically low topological indices compared with the other four $S$. 
spinosa root systems. When this abnormal specimen was omitted from the analysis of covariance, the significance of the species effect was further enhanced $\left(q_{a}: F=\right.$ 27.3, $p=4 \times 10-6 ; q_{b}: F=24.7, p=7 \times 10-6$ ). A closer look at the numbers (least significant difference test) showed that all species (except Grewia flava versus Vangueria infausta) could be separated from one another at the $5 \%$ level using either $q_{a}$ or $q_{b}$ (Tree 509 removed; Statistica post hoc tests). In particular, the two Strychnos species, tending to a herringbone structure, differed markedly from the two other species. Figure 6 demonstrates this difference in topological architecture in Strychnos cocculoides (Tree 412; upper part of figure) and Vangueria infausta (Tree 711; lower part) root systems, both shown in their metrical (left) and topological (right) reconstruction. The age of the corresponding trees was 17 and 19 years, respectively. Both topological indices had a tendency to decrease slightly with age $\left(R\left(q_{a}\right.\right.$, age $)=-0.50, R\left(q_{b}\right.$, age $\left.)=-0.28\right)$.

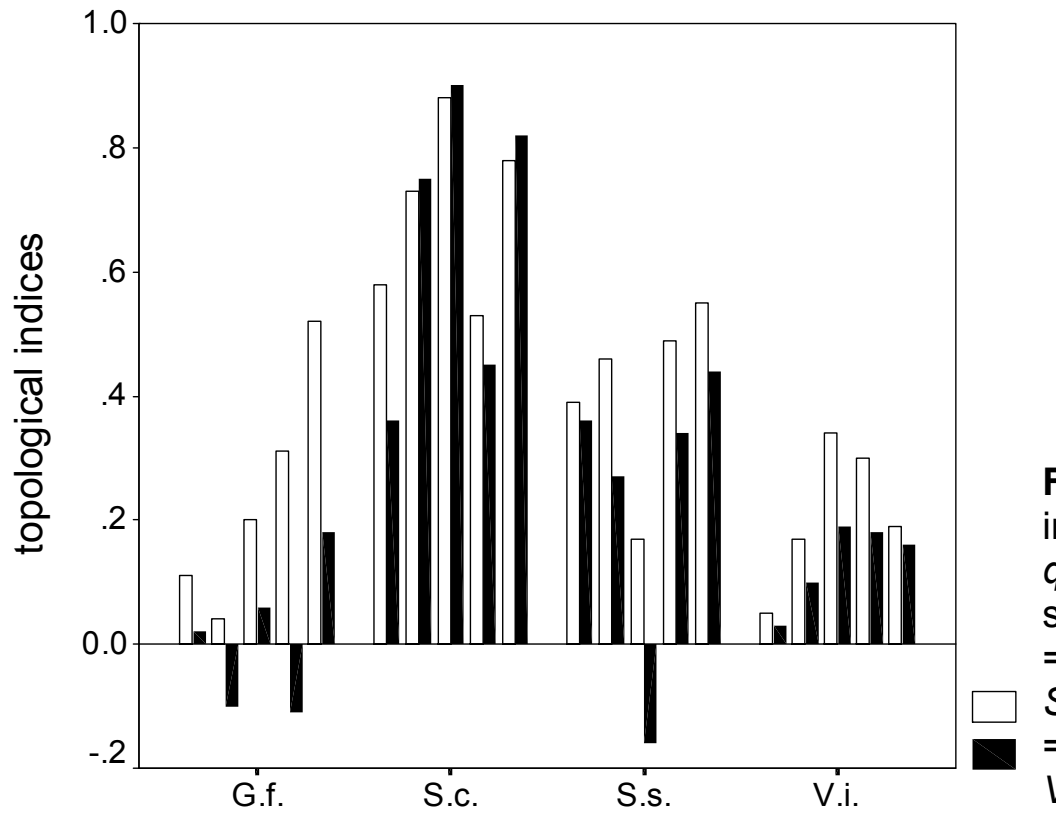

Figure 5. The topological indices $q_{a}$ (empty bars) and $q_{b}$ (black bars) of the root systems investigated. G.f. = Grewia flava, S.c. = Strychnos cocculoides, S.s. = S. spinosa, V.i. = Vangueria infausta.

As an alternative topological index, Fitter $(1985,1986,1987)$ investigated the slope that a collective of root systems exhibits in a diagram where altitude $a$ is plotted against magnitude $v_{0}$. We performed this type of analysis for the subset of the Strychnos samples contrasted to the other species (diagram not shown) and obtained a difference corroborating our finding that the two Strychnos species differed considerably in root system topology from the Grewia and Vangueria specimens that were investigated. 
Table 2. Geometrical parameters of the root systems of each sample tree.

\begin{tabular}{|c|c|c|c|c|c|c|c|c|}
\hline species & tree & $\left.c d^{1}\right)$ & $\left.r_{\max }^{2}\right)$ & $\left.L^{3}\right)$ & $\left.L_{p}{ }^{4}\right)$ & $\left.v^{5}\right)$ & $\left.I_{\mathrm{e}}{ }^{6}\right)$ & $\left.I_{i}^{7}\right)$ \\
\hline \multirow{5}{*}{$\begin{array}{l}\text { Grewia } \\
\text { flava }\end{array}$} & 203 & 97 & 5708 & 190509 & 2568 & 8304 & 851 & 641 \\
\hline & 206 & 65 & 3267 & 73076 & 1257 & 2884 & 652 & 454 \\
\hline & 213 & 58 & 3349 & 58991 & 1906 & 1769 & 614 & 562 \\
\hline & 214 & 32 & 1603 & 19723 & 1078 & 522 & 538 & 306 \\
\hline & 215 & 27 & 1506 & 8419 & 587 & 274 & 305 & 136 \\
\hline mean & & 55.8 & 3087 & 70144 & 1479 & 2751 & 592 & 419.8 \\
\hline$s d$ & & 28.21 & 1708.3 & 72410.2 & 770.4 & 3275.7 & 197.8 & 202.4 \\
\hline \multirow{5}{*}{$\begin{array}{l}\text { Strychnos } \\
\text { cocculoides }\end{array}$} & 405 & 56 & 2480 & 38247 & 1430 & 2443 & 644 & 144 \\
\hline & 409 & 67 & 4385 & 24595 & 2238 & 2932 & 920 & 326 \\
\hline & 412 & 48 & 3880 & 18930 & 2495 & 1599 & 1113 & 372 \\
\hline & 425 & 68 & 3417 & 50814 & 2428 & 4248 & 976 & 361 \\
\hline & 426 & 44 & 3020 & 20849 & 2282 & 1407 & 876 & 613 \\
\hline mean & & 56.6 & 3436 & 30687 & 2175 & 2526 & 905.8 & 363.2 \\
\hline$s d$ & & 10.85 & 739.1 & 13549.7 & 429.2 & 1145.5 & 171.4 & 167.3 \\
\hline \multirow{5}{*}{ Strychnos spinosa } & 501 & 72 & 5990 & 113735 & 2279 & 5682 & 1345 & 288 \\
\hline & 508 & 60 & 4230 & 42970 & 2737 & 3643 & 1262 & 462 \\
\hline & 509 & 40 & 1447 & 10545 & 1863 & 1011 & 769 & 517 \\
\hline & 510 & 64 & 2758 & 49307 & 1569 & 2983 & 901 & 160 \\
\hline & 511 & 44 & 2738 & 34700 & 1594 & 1487 & 768 & 245 \\
\hline mean & & 56 & 3433 & 50251 & 2008 & 2961 & 1009 & 334.4 \\
\hline$s d$ & & 13.56 & 1736.1 & 38413.9 & 497.5 & 1860.4 & 275.8 & 150.2 \\
\hline \multirow{5}{*}{ Vangueria infausta } & 706 & 140 & 5070 & 207137 & 2526 & 12440 & 718 & 394 \\
\hline & 708 & 86 & 3642 & 51750 & 1929 & 2989 & 580 & 309 \\
\hline & 711 & 48 & 1708 & 24645 & 1442 & 1282 & 412 & 255 \\
\hline & 712 & 60 & 1247 & 24825 & 895 & 1084 & 366 & 164 \\
\hline & 713 & 70 & 1547 & 34910 & 1058 & 1496 & 361 & 210 \\
\hline mean & & 80.8 & 2643 & 68653 & 1570 & 3858 & 487.4 & 266.4 \\
\hline$s d$ & & 35.91 & 1651.7 & 78198 & 666.5 & 4855.8 & 156.7 & 89.3 \\
\hline
\end{tabular}

${ }_{2}^{1} c d=$ Root collar diameter $(\mathrm{mm})$

${ }^{2} r_{\text {max }}=$ Maximal radial extension of the system (mm)

${ }^{3} L=$ Total coarse root length $(\mathrm{mm})$

${ }^{4} L_{\mathrm{p}}=$ Mean path length $(\mathrm{mm})$

${ }^{5} V=$ Total coarse root volume $\left(\mathrm{cm}^{3}\right)$

${ }^{6} l_{\mathrm{e}}=$ Mean exterior link length $(\mathrm{mm})$

${ }^{7} I_{\mathrm{i}}=$ Mean interior link length $(\mathrm{mm})$ 


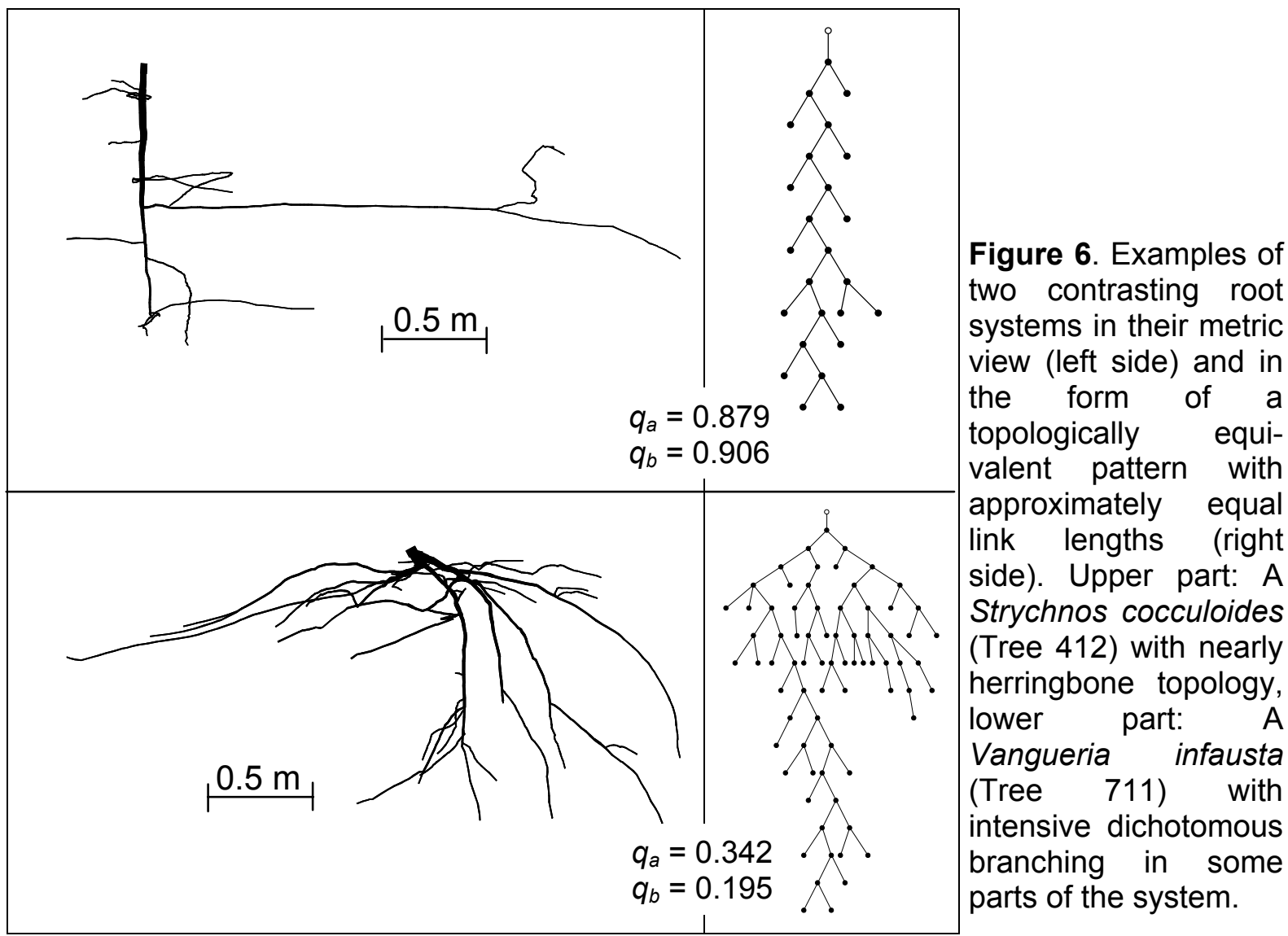

\section{Link lenghts}

The same distinction emerges for the metric parameter mean exterior link length $\left(I_{e}\right)$ (empty bars in Figure 7, cf. Table 2). An ANOVA with species as single factor yields a highly significant effect on $l_{\mathrm{e}}(F=7.31, p=0.0027)$ that becomes even more pronounced when age is included as a covariate $(F=8.44, p=0.0016)$. A post hoc test enabled a statistical separation of all species from each other at the $5 \%$ level, except for the two pairs Grewia flava versus Vangueria infausta and Strychnos cocculoides versus $S$. spinosa. In contrast, mean interior link length $\left(l_{i}\right.$; black bars in Figure 7) showed no difference between species, regardless of whether age was included as covariate $(F=0.97$ and 0.82 , respectively; $p>0.4)$. 


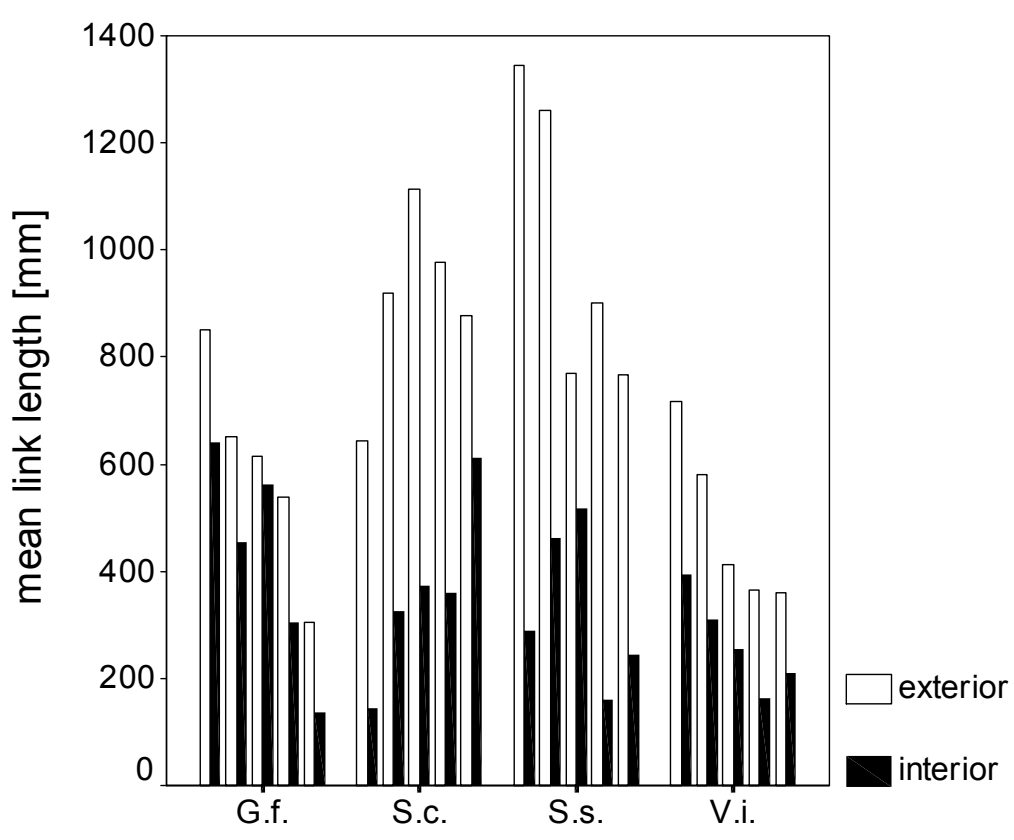

Figure 7. Mean exterior link length $l_{\mathrm{e}}$ (empty bars) and mean interior link length $l_{i}$ (black bars) for each of the root systems investigated.

\section{Leonardo's rule}

We estimated the diameter exponent from Equation 2 for each root system separately and for the whole population of branching nodes, by nonlinear regression analysis (iterative Hooke-Jeeves coordinate search, independent control with quasiNewton method; Statistica 5). The iteration converged in all cases and explained a large part of the variance (see left half of Table 3). Because the oldest and largest root segments were potentially linked to rotten parts of the system that could not be measured, we also conducted the analysis for the subsample of branching nodes where the mother segment did not exceed a threshold diameter of $20 \mathrm{~mm}$ (right-hand half of Table 3), thus focusing on the younger parts of the system. Generally, the resulting average best-fit exponent (2.29 and 2.18 for the unresticted and restricted samples, respectively) did not contradict the theoretical assumption of Leonardo's rule $(\Delta=2)$.

Adopting this value for $\Delta$, we checked linear regressions between $d^{2}$ and $\sum_{i=1}^{n} d_{i}^{2}$ for all branching nodes. These gave tight fits for all individual root systems. Statistically, it was not possible to prove or disprove that the proportionality factor $\alpha$ (cf. Equation 3 ) is 1 and the intercept 0 . However, no systematic deviation from these values was detected (see Table 4). 
Table 3. Nonlinear regression results for the diameter exponent. Abbreviation: $C D=$ coefficient of determination.

\begin{tabular}{ccccc}
\hline \multicolumn{3}{c}{ All } & \multicolumn{3}{c}{$\boldsymbol{d} \leq \mathbf{2 0}$} \\
\hline Tree & $\Delta$ & CD & $\Delta$ & CD \\
\hline 203 & 3.02 & 0.96 & 3.23 & 0.92 \\
206 & 1.98 & 0.91 & 2.58 & 0.68 \\
213 & 1.61 & 0.96 & 2.48 & 0.89 \\
214 & 1.43 & 0.51 & 2.58 & 0.68 \\
215 & 1.65 & 0.56 & 2.58 & 0.68 \\
405 & 6.27 & 0.91 & 2.65 & 0.96 \\
409 & 1.7 & 0.96 & 1.51 & 0.93 \\
412 & 1.81 & 0.96 & 3.07 & 0.93 \\
425 & 1.98 & 0.99 & 1.69 & 0.9 \\
426 & 1.58 & 0.97 & 1.43 & 0.83 \\
501 & 2.39 & 0.98 & 1.84 & 0.92 \\
508 & 2.11 & 0.98 & 2.56 & 0.9 \\
509 & 1.84 & 0.87 & 1.23 & 0.87 \\
510 & 2.38 & 0.97 & 1.91 & 0.96 \\
511 & 2.04 & 0.98 & 1.64 & 0.95 \\
706 & 2.08 & 0.98 & 2.48 & 0.92 \\
708 & 2.74 & 0.66 & 2.08 & 0.92 \\
711 & 3.65 & 0.95 & 2.21 & 0.89 \\
712 & 1.81 & 0.98 & 1.89 & 0.97 \\
713 & 1.77 & 0.99 & 1.96 & 0.92 \\
mean & $\mathbf{2 . 2 9}$ & $\mathbf{0 . 9}$ & $\mathbf{2 . 1 8}$ & $\mathbf{0 . 8 8}$ \\
$s d$ & 1.08 & 0.15 & 0.55 & 0.09 \\
\hline & & & &
\end{tabular}

\section{Length and diameter}

At the level of whole root systems, we related the root collar diameters $c d$ to the total length, $L$, of the measured roots. The value of $L$ was obtained by adding the lengths of all root segments. The linear regression of log $c d$ (independent variable) versus $\log L$ (dependent) yielded a slope of 1.95 (intercept 1.15, $r^{2}=0.75, n=20$; see Figure 8). This is close to the allometric exponent 2 proposed by van Noordwijk et al. (1994) for the relationship between base diameter and total length.

Table 4. Results of regression for Leonardo's rule. Dependent variable: $d^{2}$, independent: sum of $d^{2}$ of all daughter segments.

\begin{tabular}{lccc}
\hline species & slope & intercept & $\boldsymbol{r}^{\mathbf{2}}$ \\
\hline $\begin{array}{l}\text { Grewia } \\
\text { flava }\end{array}$ & 1.13 & -29.4 & 0.89 \\
$\begin{array}{l}\text { Strychnos } \\
\text { cocculoides }\end{array}$ & 1.00 & 17.9 & 0.90 \\
$\begin{array}{l}\text { Strychnos } \\
\text { spinosa }\end{array}$ & 1.02 & -5.7 & 0.97 \\
$\begin{array}{l}\text { Vangueria } \\
\text { infausta }\end{array}$ & 0.92 & 46.4 & 0.94 \\
mean & 1.02 & 7.3 & $\mathbf{0 . 9 3}$ \\
$\begin{array}{l}\text { sd } \\
\text { total population }\end{array}$ & 0.09 & 32.4 & 0.04 \\
\hline
\end{tabular}


Diameter can also be related to root size in topological terms. Replacing total length $L$ by the number of links $v$, we compared log $c d$ with $\log v$ (cf. Spek and van Noordwijk 1994). The resulting regression was somewhat less tight (slope 1.61, intercept $-0.98, r^{2}=0.62$ ) than in the case of $\log L$.

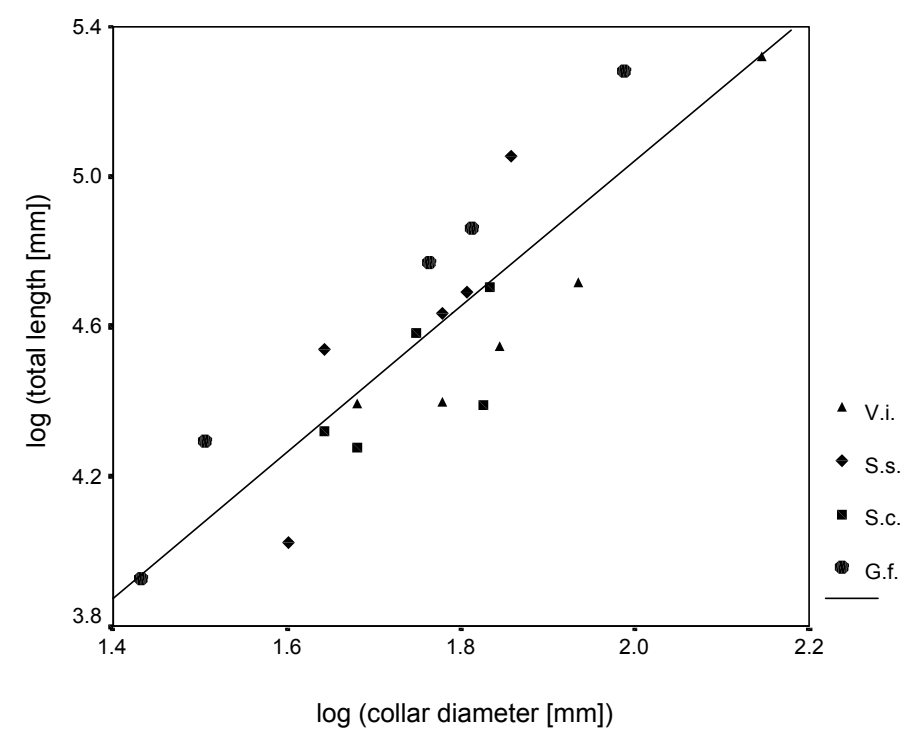

Figure 8. Logarithms of root collar diameter $(c d)$ and total coarse root length $(L)$ of all 20 investigated root systems, together with the best-fit regression line (see text).

The relationship between diameter and length can also be studied at the level of individual root segments within a system. Besides the total length $L$ distal to a given segment, we used GROGRA to calculate the mean distal pathlength $L_{p}$ (values in Table 2) and maximal distal pathlength $L_{\max }$ (not shown). Both $L_{p}$ and $L_{\max }$ typically exhibited a broad scattering when plotted against root segment diameter. Tentative nonlinear fitting of the equation $d=\gamma\left(L_{p}+I_{0}\right)^{\beta}$ from McMahon and Kronauer (1976) yielded generally unconvincing results (slow convergence; and the shape of the scatterplot was poorly reflected by the regression curve). Total distal root length $L$ showed a better correlation to segment diameter, particularly when only small diameters were considered (e.g., Figure 9).

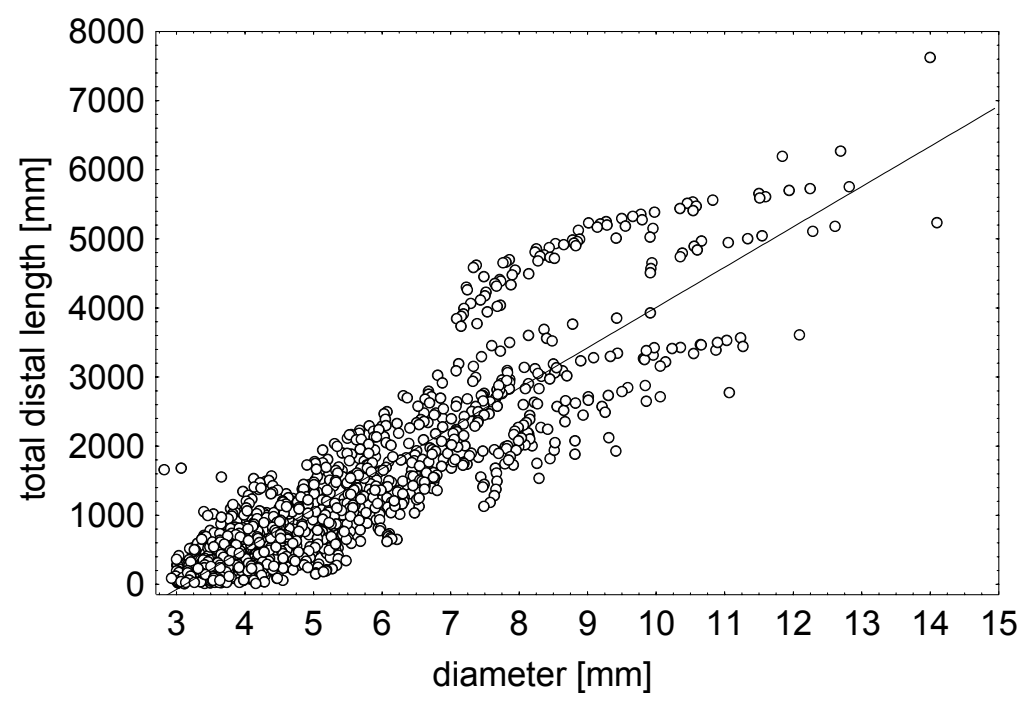

Figure 9. Diameter $d$ and total distal root length $L$ of all segments with $3<d<15 \mathrm{~mm}$ from a Grewia flava coarse root system (Tree 213). Shown also is the linear regression $L=$ $-1823+583 d\left(r^{2}=0.81, n=\right.$ 1323) for this individual root system. 
Correlation was not improved when $d$ was replaced by $d^{2}$ in the linear regression approach for $L$. Only for the largest and oldest segments was the fit better in the quadratic case.

\section{Root volume and diameter}

At the level of whole root systems, we found a good correlation between log (root collar diameter) and log (total coarse root volume), shown in Figure 10 (slope 2.19, intercept 2.47, $r^{2}=0.84, n=20$ ). The slope of the regression lines ( $\omega$ in the relation $\mathrm{V}$ $\left.=\theta d^{\omega}\right)$ was relatively stable among the four species investigated.

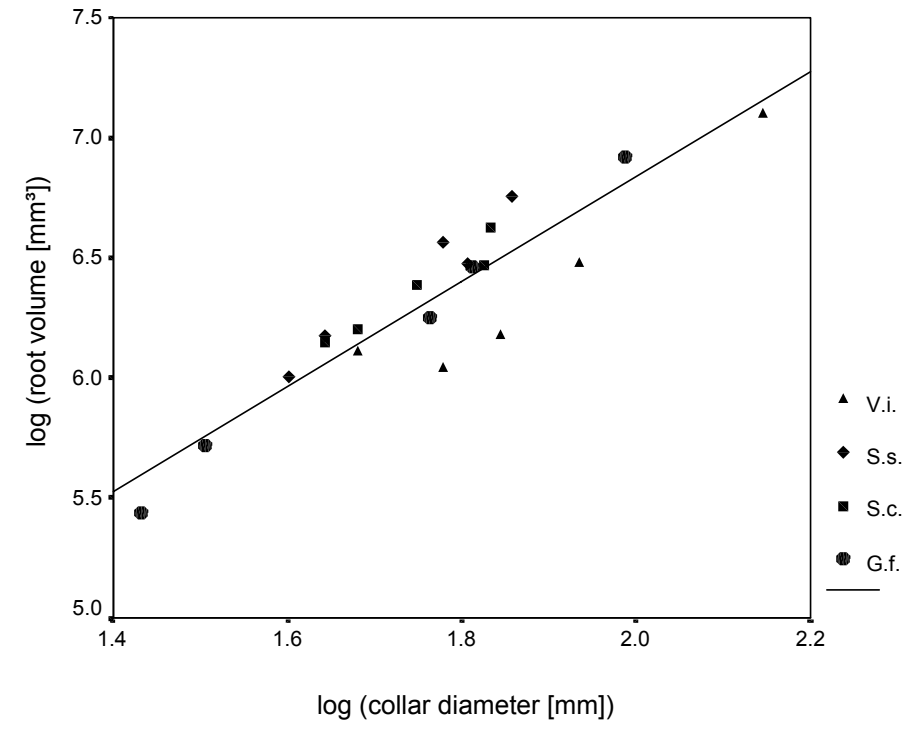

Figure 10. Logarithms of root collar diameter $(c d)$ and of total root volume $(V)$ of all 20 root systems investigated, together with the best-fit regression line (see text).

\section{Discussion}

In a methodological sense, our study - together with earlier results from the same data (Oppelt et al. 2000) - demonstrates the large amount of information that can be extracted from one architectural database by means of 3-D reconstruction and analysis. Until now, the advantages of detailed 3-D reconstruction of branching structures have mainly been appreciated in studies of aboveground plant architecture (Sinoquet and Rivet 1997, Godin et al. 1999). The diverse pieces of information are like a puzzle from which a picture, giving better insight into structural and functional aspects of plant architecture, can emerge. Furthermore, the architectura data can be the basis for simulation studies (e.g., Früh and Kurth 1999 for aboveground structures).

When our data are compared with results from the literature, our restriction to coarse roots $(d \geq 3 \mathrm{~mm})$ has to be taken into account. Most published studies on root system topology consider small seedlings in which all roots, including the finest, were measured. The topology of fine root branching could differ from the patterns found at the coarse root scale. The differences that we identified in our root systems between 
fractal dimensions at coarse root and fine root scales (Oppelt et al. 2000) indicate that such a structural gap between the two scales might exist for topology as well.

The large values of average exterior and interior link lengths in our study (ranging from $14 \mathrm{~cm}$ to $1.3 \mathrm{~m}$ ), which lie almost one order of magnitude greater than values reported by Fitter (1987) for a number of herbaceous plants and tree seedlings, are probably also due to our omission of roots $<3 \mathrm{~mm}$.

In a qualitative sense, it is well-known that a deep taproot with little lateral growth is adapted to dry environments (Epstein 1973). In quantitative simulation studies, Fitter et al. (1991) found that herringbone root systems have a higher exploitation efficiency in terms of soil volume accessed, but require more carbon for construction compared with dichotomous patterns of equal magnitude. Hence the herringbone pattern is likely to be favored when soil resources limit growth. We conjecture that in the case of our sample trees - all grown under arid conditions - the two Strychnos species are more specifically adapted to extreme drought than the other two species investigated. This is consistent with the observation that both Strychnos species exhibit a distinct xeromorphic habit in their leaf morphology, whereas Grevia flava and Vangueria infausta show less pronounced xeromorphic crown features. Root system topology and exterior link lengths thus appear to be indicators of ecophysiological differences in drought adaptation.

Because the root systems considered in this study have also been investigated for their fractal dimension $D$ (see Oppelt et al. 2000), the question arises whether $D$ is related to topological parameters. In fact, the topological index $q_{a}$ is negatively correlated with $D(r=-0.56)$ : the more a root system conforms to the herringbone pattern, the lower its space-filling potential and its fractal dimension. However, our findings also show that a considerable amount of variance of $D$ cannot be explained by purely topological properties (all other parameters calculated in this study showed a weaker correlation with $D$ than that obtained for $q_{a}$ ). Hence the fractal dimension $D$ remains a characteristic in its own right, integrating topological and geometrical properties of a whole root system (Fitter and Stickland 1992).

The diameter exponent of most of the root systems we investigated was close to the ideal value of 2 , indicating preservation of cross-sectional area in the branching nodes. Thus, application of Leonardo's rule in models of secondary growth and hydraulic function, which is quite common in the literature, is corroborated - at least for coarse roots. Given the high hydraulic conductivity of coarse roots (Riedl 1937, Fahn 1964, Lafolie et al. 1991), this amounts to a homogeneity of flow velocity throughout the system, provided there is a constant proportion of functional xylem in all coarse roots. 
Van Noordwijk et al. (1994) and, more generally, West et al. (1997) derived several scaling laws from the theoretical assumptions of self-similarity and preservation of flow. These laws were confirmed quite well when we checked them at the level of whole root systems. Total length of coarse roots is roughly proportional to crosssectional area of the root collar, as predicted by van Noordwijk et al. (1994), and a particularly stable fit was found between total volume of coarse roots and collar diameter (Figure 10), although the exponent of the diameter in this relation, 2.19, deviates slightly from the theoretical value of 2.67 predicted by West et al. (1997). Our number of replicates was too small for statistical security at the whole-system level, but nevertheless it seems possible to use root collar diameter as a predictor of total coarse root length and biomass on the basis of these scaling relations.

However, the picture becomes more complex when the lengths and diameters inside the root system are considered. Our exemplary Figure 9 shows considerable variance, but also some traces of structured patterns in the data. Simple power laws like those proposed by McMahon and Kronauer (1976) or van Noordwijk et al. (1994) seem inadequate to describe these structures. The distribution of link lengths in the root system is much more complicated than these authors assumed in their selfsimilar models. Crawford and Young (1990) suggested that, in branching systems of higher plants, a spectrum of scale factors, which itself has a fractal signature, may operate. Our data do not contradict this assumption. Our topological results suggest that the architecture of the sample systems has features that do not fit into the standard self-similar branching models. Morphological features like developmental axes, reiteration or functional differentiation of root segments may contribute to this internal complexity.

\section{Conclusions}

The architectural comparison of four species has revealed significant differences, resulting in a coherent picture: the two Strychnos species tend to have a root system topology conforming nearly to the herringbone pattern and large unbranched zones at the distal ends of their root axes. This is in accordance with the general appearance of these root systems (Figure 4; see also Oppelt et al. 2000) and indicates an explorative strategy of root system architectural development, whereas Grewia flava and Vangueria infausta, have a topology tending to dichotomous or intermediate patterns and shorter exterior links that may possibly exploit smaller volumes of soil more thoroughly.

It will be necessary, however, to complement our study by investigations of fine root distribution, because the diameter threshold of $3 \mathrm{~mm}$ prevented a direct calculation of exploited soil and total root biomass. Nevertheless, the pattern of coarse roots sets 
structural constraints on the extent and locations of fine root development, and thus has implications for resource capture and efficiency of growth.

Our empirical test of cross-sectional area preservation supported the pipe stem theory, justifying the use of this simple rule in simulation models.

Likewise, some scaling laws deduced from theoretical considerations, particularly that of West et al. (1997), were confirmed by our data and can be used to predict parameters of whole coarse root systems from root collar diameter. However, a closer consideration of the inner structure of the root systems revealed more complicated patterns that could not be expressed in simple allometric relations.

Generally, we believe that further improvements in our understanding of root system architecture, beyond the characteristics described in this study, can be obtained only if ontogenetic development is taken into account (Colin-Belgrand et al. 1989, Raimbault 1991). Root architecture results from an interplay between endogenous growth laws and opportunistic reactions (Atger 1991). Dynamic studies of growing root systems are, however, difficult and rare, particularly in the case of large trees; therefore, it makes sense to extract as much information as possible from the static data at our disposal.

\section{Acknowledgements}

We are grateful for organizational and administrative support from Georg Jentschke, Branislav Sloboda and Andrea Polle. We thank Mmoloki Botite and Golekwang Phiri for their excellent assistance in fieldwork and Gustavo Anzola Jürgenson for software adaptation. John Sperry gave valuable advice which improved the paper. Funding was provided by the Commission of the European Union under research grant INCODC (ERBIC18CT960035). Technical support from Veld Products Research \& Development was also essential and is gratefully acknowledged.

\section{References}

Anzola Jürgenson, G.A. 1998. Auswertung und Modellierung der Morphologie junger Fichten (Picea abies (L.) Karst.) in Abhängigkeit von Licht und Wuchsdichte. Diploma Thesis, Universität Göttingen, 72 p.

Atger, C. 1991. L'architecture racinaire - est-elle influencée par le milieu? In L'arbre. Biologie et Développement. Ed. C. Edelin. Naturalia Monspeliensia, Montpellier, pp 71-84.

Berntson, G.M. 1995. The characterization of topology: a comparison of four topological indices for rooted binary trees. J. Theor. Biol. 177:271-281.

Berntson, G.M. 1997. Topological scaling and plant root system architecture: developmental and functional hierarchies. New Phytol. 135:621-634. 
Bert, D., F. Danjon, D. Loustau, A. Porté, P. Trichet, I. Champion and C. Godin. 1998. Topology and geometry measurement of root and shoot architecture of Pinus pinaster. Second International Workshop on Functional-Structural Tree Models (Clermont-Ferrand, France, 12-15 Oct. 1998), Abstract Volume. INRA, ClermontFerrand, pp 7-8.

Colin-Belgrand, M., L. Pagès, E. Dreyer and H. Joannes. 1989. Analysis and simulation of the architecture of a growing root system: application to a comparative study of several tree seedlings. Ann. Sci. For. 46 (Suppl.):288s-293s.

Crawford, J.W. and I.M. Young. 1990. A multiple scaled fractal tree. J. Theor. Biol. 145:199-206.

Cruiziat, P. 1998. Structural-functional tree models (SFTms): where should we go? In Second International Workshop on Functional-Structural Tree Models, Abstract Vol., INRA, Clermont-Ferrand (France), p 94.

Deo, N. 1974. Graph theory with applications to engineering and computer science. Prentice-Hall, Englewood Cliffs, NJ, 478 p.

Epstein, E. 1973. Roots. Sci. Am. 228:48-58.

Fahn, A. 1964. Some anatomical adaptations of desert plants. Phytomorphology 14:93-102.

Fitter, A.H. 1985. Functional significance of root morphology and root system architecture. In Ecological Interactions in Soil. Eds. A.H. Fitter, D. Atkinson, D.J. Read and M.B. Usher. Blackwell, Oxford, pp 87-106.

Fitter, A.H. 1986. The topology and geometry of plant root systems: influence of watering rate on root system topology in Trifolium pratense. Ann. Bot. 58:91-101.

Fitter, A.H. 1987. An architectural approach to the comparative ecology of plant root systems. New Phytol. 106 (Suppl.):61-77.

Fitter, A.H. and T.R. Stickland. 1991. Architectural analysis of plant root systems. 2. Influence of nutrient supply on architecture in contrasting plant species. New Phytol. 118:383-389.

Fitter, A.H. and T.R. Stickland. 1992. Fractal characterization of root system architecture. Funct. Ecol. 6:632-635.

Fitter, A.H., T.R. Stickland, M.L. Harvey and G.W. Wilson. 1991. Architectural analysis of plant root systems. 1. Architectural correlates of exploitation efficiency. New Phytol. 118:375-382.

Früh, T. and W. Kurth. 1999. The hydraulic system of trees: theoretical framework and numerical simulation. J. Theor. Biol. 201:251-270. 
Gandar, P.W. and K.A. Hughes. 1988. Kiwifruit root systems. 1. Root-length densities. N.Z. J. Exp. Agric. 16:35-46.

Godin, C., Y. Guédon and E. Costes. 1999. Exploration of a plant architecture database with the AMAPmod software illustrated on an apple tree hybrid family. Agronomie 19:163-184.

Grimaldi, R.P. 1989. Discrete and combinatorial mathematics. An applied introduction. 2nd Edn. Addison-Wesley, Reading, MA, 722 p.

Hughes, K.A., P.W. Gandar and H.N. de Silva. 1995. Exploration and exploitation of soil by apple, kiwifruit, peach, Asian pear and grape roots. Plant Soil 175:301-309.

Knuth, D.E. 1973. The art of computer programming. Vol. 1. Fundamental Algorithms. 2nd Edn. Addison-Wesley, Reading, MA, 634 p.

Kurth, W. 1994. Growth grammar interpreter GROGRA 2.4. Berichte des Forschungszentrums Waldökosysteme, Vol. B 38. Universität Göttingen, 192 p.

Lafolie, F., L. Bruckler and F. Tardieu. 1991. Modeling root water potential and soilroot water transport: I. Model presentation. Soil Sci. Soc. Am. J. 55:1203-1212.

Liu, C.L. 1977. Elements of discrete mathematics. McGraw-Hill, New York, 294 p.

Long, C.A. 1994. Leonardo da Vinci's rule and fractal complexity in dichotomous trees. J. Theor. Biol. 167:107-113.

Lynch, J. 1995. Root architecture and plant productivity. Plant Physiol. 109:7-13.

MacDonald, N. 1983. Trees and networks in biological models. Wiley, Chichester, U.K., 215 p.

Mandelbrot, B.B. 1978. The fractal geometry of trees and other natural phenomena. In Geometric Problems and Biological Structures. Lect. Notes Biomath. 23:235-248.

Mandelbrot, B.B. 1983. The fractal geometry of nature. W.H. Freeman, New York, $468 \mathrm{p}$.

McMahon, T.A. and R.E. Kronauer. 1976. Tree structures: deducing the principle of mechanical design. J. Theor. Biol. 59:443-466.

Mendès France, M. 1981. De l'arbre de Leonardo da Vinci à la théorie de la dimension. Revue du Palais de la Découverte 10:52-60.

Murray, C.D. 1927. A relationship between circumference and weight in trees and its bearing on branching angles. J. Gen. Physiol. 10:725-729.

Niklas, K.J. 1994. Plant allometry. The scaling of form and process. Univ. Chicago Press, Chicago, 395 p. 
Oppelt, A.L., W. Kurth, H. Dzierzon, G. Jentschke and D.L. Godbold. 2000. Structure and fractal dimensions of root systems of four co-occurring fruit tree species from Botswana. Ann. For. Sci. 57: 463-475.

Perttunen, J., R. Sievänen, E. Nikinmaa, H. Salminen, H. Saarenmaa and J. Väkevä. 1996. LIGNUM: a tree model based on simple structural units. Ann. Bot. 77:87-98.

Raimbault, P. 1991. Quelques observations sur les systèmes racinaires des arbres de parcs et d'alignements: diversité architecturale et convergence dans le développement. In L'arbre. Biologie et Développement. Ed. C. Edelin. Naturalia Monspeliensia, Montpellier, pp 85-96.

Richter, J.P. 1970. The notebooks of Leonardo da Vinci (1452-1519), compiled and edited from the original manuscripts. Dover, New York (Reprint of a work originally published by Sampson Low, Marston Searle and Rivington, London 1883), 367 p.

Riedl, H. 1937. Bau und Leistungen des Wurzelholzes. Jb. Wiss. Bot. 85:1-75.

Shinozaki, K., K. Yoda, K. Hozumi and T. Kira. 1964. A quantitative analysis of plant form—the pipe model theory. I. Basic analyses. Jap. J. Ecol. 14:97-103.

Sievänen, R., A. Mäkelä, E. Nikinmaa and E. Korpilahti. 1997. Preface. Special Issue on Functional-Structural Tree Models, Silva Fenn. 31:237-238.

Sinoquet, H. and P. Rivet. 1997. Measurement and visualization of the architecture of an adult tree based on a three-dimensional digitising device. Trees 11:265-270.

Spek, L.Y. and M. van Noordwijk. 1994. Proximal root diameter as predictor of total root size for fractal branching models. II. Numerical model. Plant Soil 164:119-127.

Tucker, A. 1980. Applied Combinatorics. Wiley, New York, 462 p.

van Noordwijk, M., L.Y. Spek and P. de Willigen. 1994. Proximal root diameter as predictor of total root size for fractal branching models. I. Theory. Plant Soil 164:107117.

Werner, C. and J.S. Smart. 1973. Some new methods of topologic classification of channel networks. Geogr. Anal. 5:271-295.

West, G.B., J.H. Brown and B.J. Enquist. 1997. A general model for the origin of allometric scaling laws in biology. Science 276: 122-126. 
The following article

Contrasting rooting patterns of some arid-zone fruit tree species from Botswana I. Fine root distribution

contributed by:
A.L. Oppelt,
W. Kurth,
G. Jentschke,
D.L. Godbold

will be published in:

Agroforestry Systems (2004) 



\section{Contrasting rooting patterns of some arid-zone fruit tree species}

\section{from Botswana - I. Fine root distribution}

\section{Abstract}

To assess the possible degree of root competition from fruit trees which could potentially be used in agroforestry systems, fine root density of fruit trees Strychnos cocculoides BAK., Strychnos spinosa LAM. (Loganiaceae) and Vangueria infausta BURCH. (Rubiaceae), as well as from the shrubby species, Grewia flava DC. (Tiliaceae) was investigated. Vangueria infausta had the highest fine root densities in both vertical and horizontal extensions. In Vangueria infausta fine root density decreased with increasing soil depth. For the other species in the $80 \mathrm{~cm}$ soil profile investigated, no significant changes in fine root density with soil depth were found. For Strychnos cocculoides almost no fine roots were detected in the upper soil horizon $(0-20 \mathrm{~cm})$. Using fine root surface area densities, exploration and exploitation indices were calculated. Vangueria infausta had the highest value of the exploration index compared to the other species. For use in agroforestry systems Vangueria infausta was estimated to be the most competitive of the investigated species, whereas Strychnos cocculoides seems to be the less competitive. Strychnos cocculoides has additionally spatial arrangements of fine roots favourable for agroforestry, slowly increasing with depth and additionally low concentrations in upper soil layers.

\section{Introduction}

Indigenous fruit tree species are becoming an increasingly important nutritional source, and there is considerable interest in incorporating fruit trees into agroforestry systems. As fine roots are responsible for water and nutrient uptake, in arid regions with high competition between crop plants and trees for water, information about spatial fine root distribution is essential for an effective design and management of simultaneous agroforestry systems (Ong et al. 1991). In order to consider the practical consequences of interaction processes, to evaluate their suitability for managed systems (van Noordwijk et al. 1996), this information is also needed to predict the 'competitive' and 'complementary' influence of tree species.

Various approaches can be found in the literature to quantify above and belowground interactions between trees and crops (Ong et al. 1991, Daniel et al. 1991). Büsgen (1905) first distinguished "intensive" from "extensive" tree root systems, according to the extent of soil volume exploited. The question has arisen how species-related differences, which are easy to observe but difficult to quantify, can be described with useful parameters. In this context two most commonly used notions - "exploration" 
and "exploitation"' - were developed to describe different distribution patterns of root growth. These terms were coined to gain valuable information on tree root systems, to quantify different strategies for acquisition of belowground resources and to characterize potential competition as well as complementarity between trees and annual crops. However, their definition is not trivial and definitions often vary.

Fitter (1985) and co-worker (Fitter and Stickland 1991) assessed exploration and exploitation efficiency of root systems with different topological structures in simulation experiments. Berntson (1994) presented a model based on sizedependent and size-independent aspects of root system architecture in terms of depletion volume. Van Noordwijk et al. (1996) defined lengths of the longest (deepest) root as a rough indicator of exploration and the total length or surface area of live roots as a parameter quantifying exploitation.

In the work presented, fine root distributions of in situ grown fruit trees Strychnos cocculoides BAK., Strychnos spinosa LAM. (Loganiaceae) and Vangueria infausta BURCH. (Rubiaceae), as well as from the shrub Grewia flava DC. (Tiliaceae) were analysed. Motivated by the work of Gandar and Hughes (1988) and Hughes et al. (1995) we have developed their exploration and exploitation index, in order to describe species-dependent differences in rooting strategies. The species investigated in this work produce fruit and are important to rural people, especially those practising subsistence dryland farming. These indigenous fruit trees yield crops even in poor rainfall years when arable agriculture fails, improving food security for rural households.

Here we present the results concerning fine roots. In Part II, exploration and exploitation indices of coarse root architecture and relations between coarse and fine root distribution are presented.

\section{Material and Methods}

\section{Site description and sample trees}

The investigation site was located on sandveld near Mogorosi (Serowe Region, Central District, Botswana) between longitude $26^{\circ} 36.26^{\prime}$ and $26^{\circ} 36.70^{\prime} \mathrm{E}$ and latitude from $22^{\circ} 25.09^{\prime}$ to $22^{\circ} 25.30^{\prime}$ S, i.e. samples were located within an area of about $1.4 \mathrm{~km}^{2}$. The analysed species were growing on a "Typic Torripsamment" (cf. Soil Survey Staff 1999) with a low fertility status and a poor water holding capacity. Summer rainfall with low precipitation rates $-323,471\left[\mathrm{~mm}^{*}\right.$ year $\left.{ }^{-1}\right]$ within the years 1997 and 1998 respectively - are characteristic for this region.

Sample trees were naturally grown in an untilled habitat. A threshold between coarse and fine roots was set at $3 \mathrm{~mm}$, because a reconstruction of the original position of 
coarse roots below this value would not have been possible. Each species was represented by five replicates. Analysis of growth rings at the root collar showed that the sample trees have a wide range of ages: Grewia flava 13-25 years, Strychnos cocculoides 13-29 years, Strychnos spinosa 12-20 years and Vangueria infausta 1936 years (Oppelt et al. 2000). With the exception of Vangueria infausta all species have xerophytic leaf structures.

\section{Fine root sampling}

Before manual excavation of the coarse root systems (Oppelt et al. 2000, see also Part II), systematic fine root samples were taken with a soil auger (core tube $80 \mathrm{~mm}$ in diameter and $200 \mathrm{~mm}$ in length, with a 11 volume). Four horizontal lines (N-S, NESW, E-W and SE-NW, i.e., delimiting eight sections of $45^{\circ}$ ), crossing each other at the root collar, were determined with a compass. Along each line, samples were taken at a horizontal distance of 1,2 , and $3 \mathrm{~m}$ from the tree center and up to a depth of $80 \mathrm{~cm}$ (four cores). Thus, independent of the size of each tree, 96 fine root samples were taken per individual.

Fine roots were hand-sorted from the soil cores by means of dry sieving, visually inspected to remove fine roots from other species as well as coarse roots from the investigated and other individuals. Fine roots from Grewia flava are dark black and those from Vangueria infausta are distinctively red, roots from these species were easily identified and separated. Fine roots of Strychnos species are moderately thick, approximately $1 \mathrm{~mm}$, and were easily seen. With the exception of grass roots, which could be clearly distinguished and easily separated, fine roots from other tree species seldom occurred in the rooting zones of our sample trees. Cores were sieved through a consecutive set of sieves with aperture sizes ranging from $2 \mathrm{~mm}$ to $0.1 \mathrm{~mm}$, in order to avoid loss of fine roots. Through this intensive sieving, the very fragile dead roots were completely destroyed and excluded from our samples. Due to the wide distance between the excavated specimens and because trees of the same species had a scattered distribution at the investigated sites, the probability of getting fine roots from other individuals of the same species was considered negligible. Before dry weight from all fine root samples was determined, randomly chosen samples, at least 15 per individual, if available, were stored in isopropanol for digital image analysis. Dry weight was determined after the roots had reached a constant weight at $70^{\circ} \mathrm{C}$.

\section{Digital image analysis of fine root samples}

Digital image analysis with a flat bed scanner (HP 4c) and the software WinRHIZO 3.10b (http://www.regent.qc.ca/) was applied to estimate the parameters root volume, surface area, root length and average diameter. The root samples were then dried 
and the relationship between dry weight and the above parameters determined. As the core tube contained one litre of soil, the obtained sums of root length per sample were multiplied by 10 for estimation of root length density, $R L D\left[\mathrm{~m} \mathrm{~m}^{-3}\right]$, while the root surface area $(R A D)$ had to be divided by 10 to obtain SI-units $\left[\mathrm{m}^{2} \mathrm{~m}^{-3}\right]$.

\section{Exploration and exploitation indices of fine root samples}

In contrast to other investigations calculating root-length densities $(R L D)$ in order to quantify rooting patterns, e.g. Gandar and Hughes (1988), Hughes et al. (1995), we decided to use root surface area densities (RAD) instead. This decision was justified by two reasons. As our results from linear regression yielded far better correlations between dry weight (Dwt) and surface area than between dry weight and length of fine root samples, the use of $R A D$ leads to more reliable calculations. Furthermore, in terms of nutrient and water uptake, we consider the root surface area as a more suitable physiological parameter than the root length. For example this parameter is used for a normalized measure of root conductance (Nardini et al. 2000). Fine root dry weight and linear regression results from digital image analysis were used to calculate root surface area density $(R A D)$ for each sample, based on regressions obtained for each species separately. For calculation of mean values for fine root dry weight and RAD cores without fine roots were included. In analogy to Gandar and Hughes (1988) and Hughes et al. (1995), an exploitation index $E(\phi)$, defined as the proportion of soil volume which contains roots at $R A D \geq \phi$, where $\phi$ is an arbitrary threshold, was estimated for the investigated species. $E(0)$ is the proportion of soil volume which is explored by any roots (i.e., $R A D>0$ ) and is called exploration index.

Contrary to Hughes et al. (1995), where independent of the size of coarse root systems a fixed 'standard soil volume' was used, we determined an 'individual soil volume', based on the spatial extension of coarse roots and the coring depth $(80 \mathrm{~cm})$ (cf. Oppelt et al. 2000, 2001). Based on the horizontal projection, the individual coarse root systems were divided into four quadrants along the separating directions $\mathrm{N}-\mathrm{S}$ and $\mathrm{W}-\mathrm{E}$. Within each quadrant one coarse root with the maximum extension was selected and its outermost $X$ and $Y$ coordinates were used to calculate the area of the bounding rectangle of the quadrant. The 'individual soil volume' (ISV) was defined as the sum of the four bounding rectangles, multiplied by coring depth. By considering the occupation of each quadrant separately, we obtained a gross measure of occupied volume taking spatial heterogeneity into account, and at the same time easier to determine than more refined parameters like e.g. the volume of the convex hull. Of course, our 'individual soil volume' is not rotation invariant. Based on this calculation we determined a theoretical number of core samples that are located within the ISV occupied by coarse roots. Within this coarse root space, we calculated or estimated, as far as not otherwise stated, mean values of fine root 
parameters Dwt and RAD. Thus, based on core sampling, we obtained information about explored soil volume and how it is exploited within our restriced soil volume. Theoretical (estimated) RAD values obtained from correlation of dry weight with parameters from the digital image analysis were used to enlarge the data set on which the calculations of $E(0)$ and $E(\phi)$ are based.

As the chance of containing roots increases with increasing sampler size, it has to be mentioned that values for $E(\phi)$ and $E(0)$ are relative indices which depend on the volume of cores. Our cores were one litre. Statistical analysis was done with the help of the Statistica 6.0 software (http://www.statsoft.com/).

\section{Results}

\section{Relationship between morphological parameters}

In an attempt to extrapolate the fine root dry weights to other morphological parameters, the root volume, surface area, root length, and average diameter, estimates obtained from digital image analysis were compared to the root dry weights (Table 1). No a-priori partitioning into diameter classes was applied. For all species the best correlations were found between dry weight and root volume. A less tight, but still suitable, correlation was found between dry weight and surface area. Root length was, due to heterogeneous diameter distribution, only poorly correlated to dry weight, except for Vangueria infausta. The correlation between fine root surface area and dry weight was subsequently used for extrapolation of root surface area density $R A D\left[\mathrm{~m}^{2} \mathrm{~m}^{-3}\right]$ per soil volume.

Table 1. Linear regression for fine root biomass (independent variable) vs. other root characteristics (dependent variables) obtained by digital image analysis (dependent variable $=a+b^{*}$ weight) of four arid-zone fruit tree species from Botswana.

\begin{tabular}{|c|c|c|c|c|c|c|c|c|c|c|c|c|c|c|}
\hline \multirow[t]{2}{*}{ species } & \multirow[t]{2}{*}{$\mathbf{N}$} & \multirow[t]{2}{*}{$\mathbf{R} \%$} & \multicolumn{3}{|c|}{$\begin{array}{c}\text { root volume } \\
{\left[\mathrm{cm}^{3}\right]}\end{array}$} & \multicolumn{3}{|c|}{$\begin{array}{c}\text { surface area } \\
{\left[\mathrm{cm}^{2}\right]}\end{array}$} & \multicolumn{3}{|c|}{$\begin{array}{l}\text { total length } \\
{[\mathrm{cm}]}\end{array}$} & \multicolumn{3}{|c|}{$\begin{array}{l}\text { aver. diameter } \\
{[\mathrm{cm}]}\end{array}$} \\
\hline & & & $b$ & $a$ & $r^{2}$ & $b$ & $a$ & $r^{2}$ & $b$ & a & $r^{2}$ & $b$ & $a$ & $r^{2}$ \\
\hline $\begin{array}{l}\text { Grewia } \\
\text { flava }\end{array}$ & 35 & 38 & 1.16 & 0.02 & 0.95 & 38.7 & 1.9 & 0.87 & 86.0 & 16.1 & 0.25 & 0.19 & 0.06 & 0.19 \\
\hline $\begin{array}{l}\text { Strychnos } \\
\text { cocculoides }\end{array}$ & 26 & 87 & 2.63 & -0.01 & 0.97 & 43.8 & 1.9 & 0.79 & 22.1 & 14.1 & 0.02 & 0.87 & 0.05 & 0.77 \\
\hline $\begin{array}{l}\text { Strychnos } \\
\text { spinosa }\end{array}$ & 47 & 98 & 1.52 & 0.03 & 0.88 & 39.6 & 2.7 & 0.63 & 41.7 & 17.2 & 0.04 & 0.50 & 0.06 & 0.52 \\
\hline $\begin{array}{l}\text { Vangueria } \\
\text { infausta }\end{array}$ & 82 & 30 & 2.02 & 0.03 & 0.90 & 160.1 & 2.0 & 0.84 & 1045.3 & 13.6 & 0.73 & 0.00 & 0.05 & 0.00 \\
\hline Total & 190 & 43 & 1.95 & 0.02 & 0.88 & 140.9 & 1.4 & 0.73 & 890.6 & 8.1 & 0.60 & 0.05 & 0.06 & 0.02 \\
\hline $\begin{array}{l}D w t \\
N \\
R \%\end{array}$ & ta & $f$ & I co & es cont & aining $f$ & fine ro & & & & & & & & \\
\hline
\end{tabular}


Obvious differences in the correlations revealed species-dependent characteristics: In Vangueria infausta fine root dry weight was more strongly determined by fine root length, whereas in the two Strychnos species it was to a greater degree determined by average diameter (cf. Table 1 and Figure 1).

\section{Differences between the species}

If only core samples containing fine roots were considered (including samples from outside the ISV), highest mean values of fine root dry weight per species were found for Strychnos cocculoides (60 mg Dwt $\mathrm{I}^{-1}, s d=83.8, N=30$ ) and for Vangueria infausta (70 mg Dwt l-1 $, s d=75.2, N=275$ ). In contrast, Grewia flava (30 mg Dwt l-1, $s d=68.3, N=91$ ) and Strychnos spinosa (36 mg Dwt $\left.{ }^{-1}, s d=44.5, N=48\right)$ had similar lower mean dry weight values, which were significantly different from those of Vangueria infausta and Strychnos spinosa (ANOVA, $\alpha=0.05$ ). But in terms of fine root surface area $(R A D)$, Vangueria infausta, with significantly higher mean values $\left(16.0 \mathrm{~cm}^{2} \mathrm{I}^{-1}\right)$, could be separated from all the other species (values between 3.7 and $\left.4.2 \mathrm{~cm}^{2} \mathrm{I}^{-1}\right)$. Thus, considering only samples from digital analysis $(N=190)$, fine root surface area from Vangueria infausta exceeded mean values from all other investigated species by factors between 3.8 and 4.3. However, in further analysis means were also calculated using all samples including void cores. Furthermore, a distinction between samples either from the $I S V$ or including all cores (i.e., $R A D \geq 0$ ) was made.

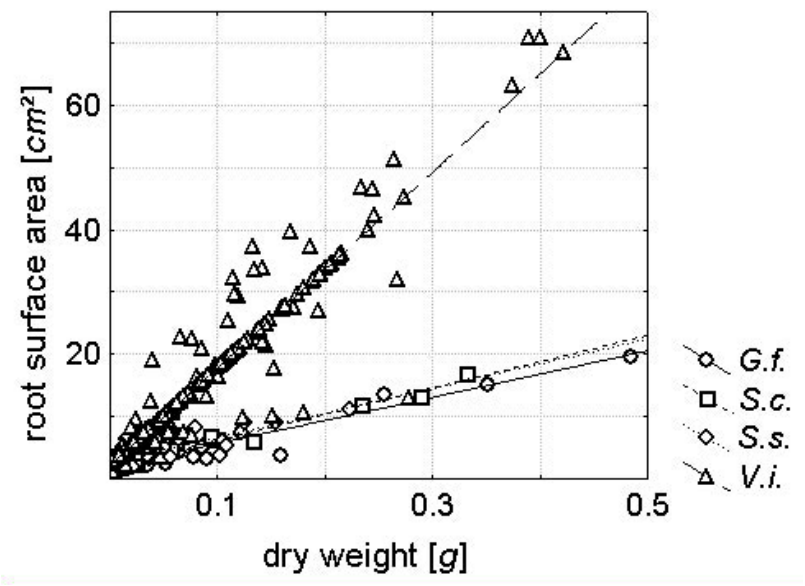

Figure 1. Fine root weight vs. fine root surface area with linear regression lines of four arid-zone fruit tree species from Botswana (G.f.: Grewia flava, S.c.: Strychnos cocculoides, S.s.: Strychnos spinosa, V.i.: Vangueria infausta).

Using samples from the ISV only, for both parameters (Dwt and RAD), Vangueria infausta could be clearly separated from the other species (ANOVA, $\alpha=0.05$ ). Fine root dry weight was much higher (factor 4.2 - 6.9) compared to the other species. In terms of fine root surface area, Vangueria infausta exceeded that of Grewia flava by a factor of 8 and both Strychnos species by factors of around 13. Similar results were obtained when core samples from outside the individual soil volume were included in 
our analysis. Based on these data alone, Vangueria infausta has to be considered as potentially the most competitive (cf. Table 2).

Table 2. Mean and maximum values from digital image analysis for total length, surface area and volume of fine root samples for some arid-zone fruit tree species from Botswana.

\begin{tabular}{|c|c|c|c|c|c|c|c|c|c|c|c|c|c|}
\hline \multirow[t]{2}{*}{ species } & \multirow[t]{2}{*}{$\mathbf{N}$} & \multicolumn{3}{|c|}{$\begin{array}{l}\text { root volume } \\
{\left[\mathrm{cm}^{3} \mathrm{dm}^{-3}\right]}\end{array}$} & \multicolumn{3}{|c|}{$\begin{array}{l}\text { surface area } \\
{\left[\mathrm{cm}^{2} \mathrm{dm}^{-3}\right]}\end{array}$} & \multicolumn{3}{|c|}{ 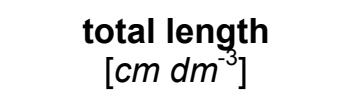 } & \multicolumn{3}{|c|}{$\begin{array}{c}\text { aver. diameter } \\
{[\mathrm{cm}]}\end{array}$} \\
\hline & & mean & $\max$ & $s d$ & mean & $\max$ & $s d$ & mean & $\max$ & $s d$ & mean & $\max$ & $s d$ \\
\hline $\begin{array}{l}\text { Grewia } \\
\text { flava }\end{array}$ & 35 & 0.09 & 0.52 & 0.12 & 4.2 & 19.7 & 2.6 & 21.0 & 59.0 & 17.6 & 0.07 & 0.21 & 0.04 \\
\hline $\begin{array}{l}\text { Strychnos } \\
\text { cocculoides }\end{array}$ & 26 & 0.10 & 0.68 & 0.14 & 3.7 & 11.7 & 2.2 & 15.0 & 38.1 & 9.0 & 0.08 & 0.24 & 0.05 \\
\hline $\begin{array}{l}\text { Strychnos } \\
\text { spinosa }\end{array}$ & 47 & 0.08 & 0.33 & 0.07 & 4.1 & 11.2 & 16.0 & 18.8 & 41.4 & 9.0 & 0.07 & 0.17 & 0.03 \\
\hline $\begin{array}{l}\text { Vangueria } \\
\text { infausta }\end{array}$ & 82 & 0.20 & 0.95 & 0.20 & 16.0 & 70.7 & 4.2 & 104.5 & 418.5 & 108.5 & 0.05 & 0.14 & 0.02 \\
\hline
\end{tabular}

$\mathrm{N}$ number of samples analysed by digital image analysis

sd standard deviation

\section{Influence of tree age}

As core samples from the investigated species originated from individuals of different age classes, the question arises whether ontogenetic aspects influence mean fine root dry weight and fine root surface area. Except for Grewia flava and Strychnos cocculoides, which showed a negative influence of age on mean fine root Dwt respectively $R A D$, the other species showed slightly positive correlations between age and fine roots (samples from ISV). These correlations did even deteriorate when all samples were included in our analysis. However, due to the fact that degrees of determination were always low and that the investigation is based on a small number of individuals, a clear influence of age on Dwt respectively $R A D$ could not be determined. In a regression analysis (applied to samples from one species) with fine root dry weight or $R A D$ as dependent variables and age as the independent variable, clear significant regression coefficients for age were determined only for Vangueria infausta and Grewia flava. Hence the age of the rootstocks can generally not be used to predict mean dry weight or surface area of fine root samples. This is in contrast to results obtained by Hughes et al. (1995) on smaller crop plants, and with fixed standard soil volume.

\section{Spatial distribution of fine roots}

In order to test for species-dependent differences of vertical and horizontal fine root density, one-factorial ANOVA tests between single species were carried out. With or without the inclusion of covariates (distance and depth), all species could be statistically separated from Vangueria infausta $(\alpha=0.05)$. The vertical distribution of 
mean $R A D$ for the four species is shown in Figure 2. For the RAD, Vangueria infausta always showed significantly higher values independent of the considered profile depth (ANOVA, $\alpha=0.05$ ). For the other species no significant changes in $R A D$ with soil depth could be shown. In the case of Strychnos cocculoides, apart from one core sample located outside the ISV, no fine roots could be detected in the upper soil horizon $(0-20 \mathrm{~cm})-c f$. Figure 2 and Figure 3.

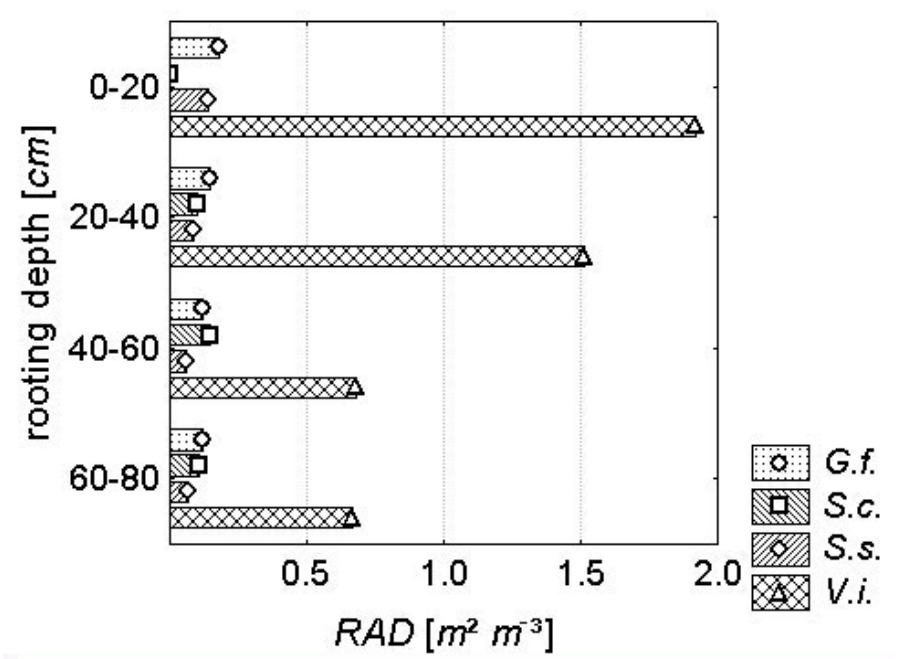

Figure 2. Vertical distribution of mean fine root density for the ISV expressed as root area density $(R A D)$ of four aridzone fruit tree species from Botswana (G.f.: Grewia flava, S.c.: Strychnos cocculoides, S.s.: Strchnos spinosa, V.i.: Vangueria infausta).

The horizontal distribution of fine roots also showed some species-dependent differences at $0-80 \mathrm{~cm}$ soil depth. If we consider only core samples from the ISV, fine root densities (RAD) and their standard deviations decreased with horizontal distance. In terms of RAD Vangueria infausta samples had always significantly higher values than those from the other species independent of their distance (ANOVA, $\alpha=0.05$ ). Hence, horizontal fine root density of Vangueria infausta greatly exceeded those of the other species at all distances from the root collar at a depth of $0-80 \mathrm{~cm}$ (cf. Figure 3).

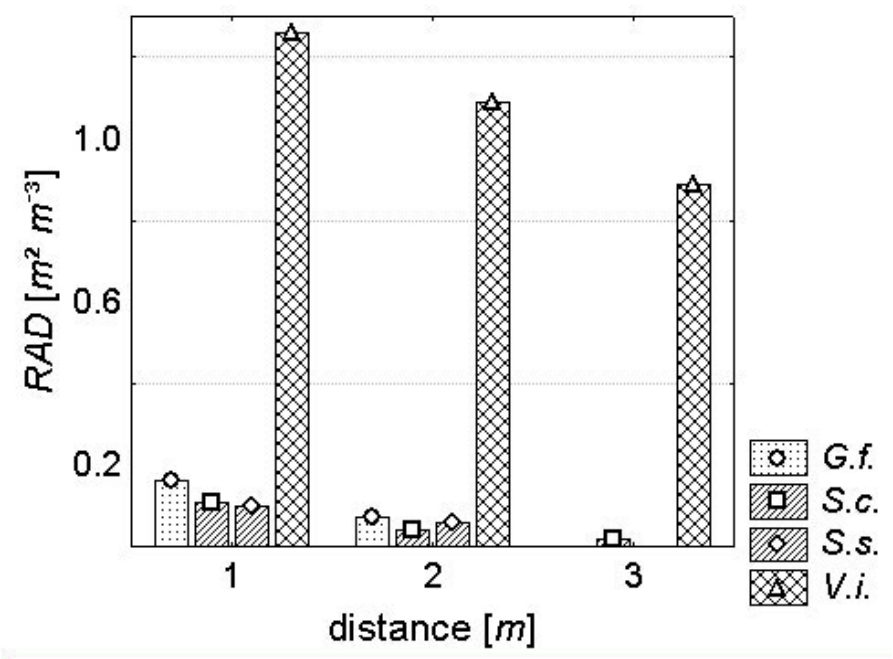

Figure 3. Horizontal distribution of mean fine root density for the ISV expressed as root area density $(R A D)$ of four arid-zone fruit tree species from Botswana (G.f.: Grewia flava, S.c.: Strychnos cocculoides, S.s.: Strchnos spinosa, V.i.: Vangueria infausta) for rooting depth $0-80 \mathrm{~cm}$ and horizontal distance 1-3 m. 
In the upper soil layers (0-20 and 20-40 cm) Vangueria infausta showed high fine root densities with, in comparison to the other species, the most pronounced decrease with horizontal distance from the stem (Figure 4). Also here, compared to the other species and independent of horizontal distance, mean RAD values from Vangueria infausta samples revealed significant differences for the upper soil layers. The relative $R A D$ values for the top soil layer $(0-20 \mathrm{~cm})$, did not vary greatly between the species Grewia flava (31\%), Strychnos cocculoides (0\%), Strychnos spinosa $(38 \%)$ and Vangueria infausta (40\%). The inclusion of deeper soil layers $(0-40 \mathrm{~cm})$, did not influence the variation between the species (Grewia flava 58\%, Strychnos spinosa $63 \%$ and Vangueria infausta $72 \%$ ). Here, too, Strychnos cocculoides showed the lowest values $(33 \%)$ in terms of $R A D$. In all cases differences between the species were always more pronounced when $R A D$ was considered in comparison to Dwt.

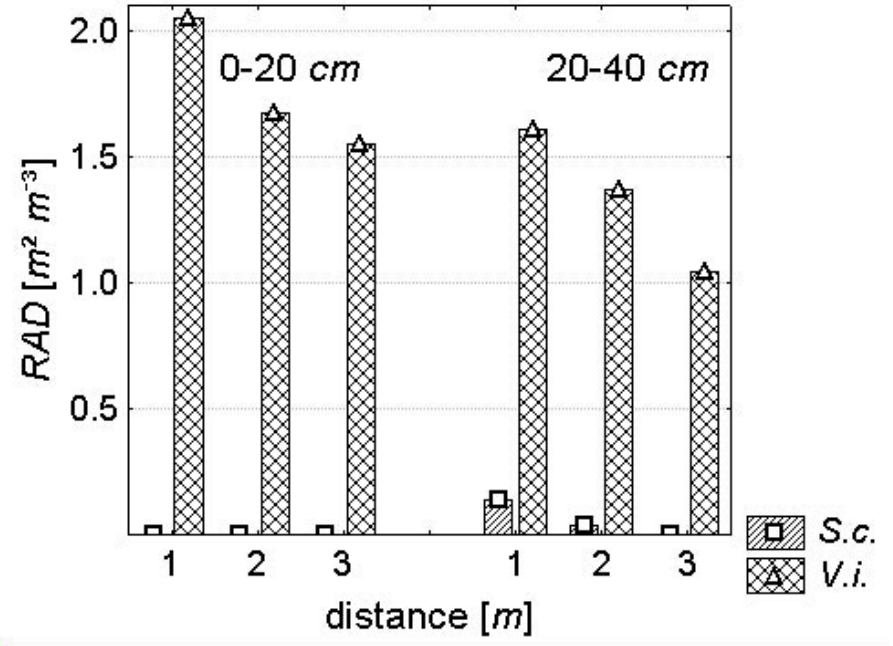

Figure 4. Horizontal distribution of mean fine root density expressed as root area density $(R A D)$ from the most contrasting species Strychnos cocculoides (S.c.) and Vangueria infausta (V.i.) from ISV for different rooting depths $(0-20 \mathrm{~cm}$ : left bars, respectively $20-40 \mathrm{~cm}$ : right bars) and varying distances (1-3 $\mathrm{m})$ from the root collar.

\section{Soil exploration and exploitation assessed by fine root samples}

Results of exploration indices, $E(0)$, calculated as the proportion of core samples containing fine roots (within the ISV) revealed species-related differences. Calculation of $E(0)$ for each species showed that Vangueria infausta had always a higher mean value of exploration index compared to the other species. Furthermore, Strychnos cocculoides with considerably lower mean values for $E(0)$ could be statistically separated from the remaining species. For example, considering a rooting depth between 0 and $80 \mathrm{~cm}, 81 \%$ of the Vangueria infausta samples taken from the individual soil volume contained fine roots. Values of $E(0)$ for Grewia flava and Strychnos spinosa samples were already considerably lower $(41 \%$ and $21 \%$ respectively), the lowest value was shown by Strychnos cocculoides (17\% of the cores included fine roots), indicating the lowest exploration ability (cf. Table 3). Looking at these data with respect to their spatial variability, species-dependent 
differences are even more pronounced. Whereas Grewia flava and Strychnos spinosa showed the lowest variability for different soil depth $( \pm 2 \%$ respectively \pm $6 \%)$, exploration indices from Vangueria infausta ( $\pm 10 \%)$ decreased with increasing soil depth, while samples from Strychnos cocculoides ( $\pm 16 \%$ ) showed a contrasting trend. A clear ranking between the species, especially for the top soil layers $(0-40$ $\mathrm{cm}$ ), was obvious (S. cocculoides $<$ S. spinosa $<$ G. flava $<<$ V. infausta). Differences between the investigated species in mean $E(0)$ are less clear with increasing depth. However, exploration indices from Vangueria infausta have always shown significantly higher values for a soil depth between 0 and $40 \mathrm{~cm}$. At deeper soil layers, Vangueria infausta showed statistically higher mean values for $E(0)$ than all other species, with the exception of Grewia flava at 0-20 and 60-80 depth. In the case of Strychnos cocculoides values from soil layers between 0 and $40 \mathrm{~cm}$ differed significantly from all values of Vangueria infausta samples, due to considerably lower mean values.

Table 3. Mean values for exploration index $E(0)$ and parameters for exploitation index $E^{*}(\phi)$ expressed as $k$ value) in the exponential model obtained from fine root samples from Botswana.

\begin{tabular}{lcccccccc}
\hline species & $\boldsymbol{N}_{\text {Isv }}$ & $\boldsymbol{n ( 0 )}$ & $\boldsymbol{E ( 0 )}$ & $\left.\boldsymbol{s d}^{\mathbf{1}}\right)$ & $\boldsymbol{N}_{\phi}$ & $\boldsymbol{- k}$ & $\left.\boldsymbol{s d}^{\mathbf{2}}\right)$ & $\boldsymbol{r}_{\boldsymbol{m}, \boldsymbol{p}}$ \\
\hline $\begin{array}{l}\text { Grewia } \\
\text { flava }\end{array}$ & 96 & 39 & 0.41 & 0.40 & 91 & 0.32 & 1.76 & 0.92 \\
$\begin{array}{l}\text { Strychnos } \\
\text { cocculoides }\end{array}$ & 104 & 18 & 0.17 & 0.02 & 30 & 0.23 & 1.27 & 0.97 \\
$\begin{array}{l}\text { Strychnos } \\
\text { spinosa }\end{array}$ & 108 & 23 & 0.21 & 0.07 & 48 & 0.33 & 0.38 & 0.97 \\
$\begin{array}{l}\text { Vangueria } \\
\text { Infausta }\end{array}$ & 176 & 143 & 0.81 & 0.23 & 275 & 0.07 & 0.35 & 0.99 \\
\hline
\end{tabular}

$N_{\text {ISV }}$ total number of samples from individual soil volume (ISV)

$n(0)$ number of samples containing fine roots within the ISV

$E(0) \quad$ quotient $n(0) / N_{I S V}$

$s d^{1}$ ) standard deviation for $E(0)$ for all individuals of same species

$N_{\phi} \quad$ number of samples with an arbitrary value $\leq \phi$

$-k$ exponent of fitting equation, see text, for a range of $R A D: 1-60 \mathrm{~cm}^{2} \mathrm{I}^{-1}$

$\left.s d^{2}\right) \quad$ standard deviation for $E^{*}(\phi)$ with all individuals pooled

$r_{m, p}^{2} \quad$ coefficient of determination of the relation measured $v s$. predicted

In terms of potential competition with co-existing crop plants, expressed as relation of core samples within the ISV containing fine roots in different soil layers (values from all individuals per species were pooled), our investigation revealed the following results. Whereas in the case of Strychnos cocculoides no cores containing fine roots were found in the top soil layer $(0-20 \mathrm{~cm})$, the remaining species did not show pronounced differences (Grewia flava: $26 \%$, Strychnos spinosa: $26 \%$ and Vangueria infausta: 28\%). Extending this to soil layers between 0 and $40 \mathrm{~cm}$, the above mentioned differences between the species are still obvious: only $17 \%$ of all fine root containing samples are present here in the case of Strychnos cocculoides. In the 
case of the remaining species approximately half of the cores contained fine roots for the depth between 0 and $40 \mathrm{~cm}$ - Grewia flava (49\%) Strychnos spinosa (52\%) and Vangueria infausta (54\%). When we took all (i.e., also including samples from outside ISV) fine root containing samples into account, the relationship between the species did not change drastically. Considering the portion of cores with $R A D>0$, all species, except Strychnos cocculoides, had quite similar percentage of fine root containing cores for a soil depth between 0 and $40 \mathrm{~cm}$.

As individual values of $E(0)$ showed considerable variation, especially for Grewia flava and Vangueria infausta (cf. Table 3), the influence of ontogenic development was estimated. An analysis of regression with age as independent and $E(0)$ as dependent variable was carried out. The results showed a significant influence of root stock age on $E(0)$ for all individuals $\left(p=1.3 \times 10^{-5}, r^{2}=0.28\right)$. In contrast, in a similar analysis no influence of the season when the samples were taken $(p=0.20)$ could be detected. Thus, similar to the investigation of Hughes et al. (1995), our analysis confirms the dependency between $E(0)$ and root stock age.

For calculation of the exploitation index, all fine root samples, i.e. also those which originated from outside the ISV, were taken into account because a larger database was necessary for this purpose. A transformation $E^{\star}(\phi)=E(\phi) / E(0)=n(\phi) / n(0)$, where $n(\phi)$ is the number of core samples with $R A D \geq \phi$ and $n(0)$ is the number of root containing core samples, was carried out to normalise the exploitation index. By nonlinear least-square regression, a coefficient $k$ was obtained to describe the dependency between $\phi$ and $E^{*}(\phi)$ in the form $E^{*}(\phi)=e^{-k \phi}$ (Hughes et al., 1995). High values for $k$ imply a rapid decline in $E^{*}(\phi)$ for increasing threshold values $\phi$. Consequently, low $k$-values indicate a wide range of $\phi$ and therefore higher mean values of $R A D$ with a more intensive exploitation of a considered soil volume (cf. Table 3 and 5).

As minimum and maximum values for $R A D$ between the species did vary in a wide range, we could compare the species only for a common range of $R A D$. Independent of any restriction or considering only a common range of $\phi$-values, all of the investigated species could be clearly separated in terms of the $k$ value from Vangueria infausta. Additionally, only looking to common $\phi$-values, another significant difference could be detected between Grewia flava and Strychnos cocculoides. Differences were least pronounced in comparison to Grewia flava $(p=0.013)$, while the differences between Vangueria infausta and Strychnos cocculoides $(p=0.009)$ and S. spinosa ( $p=0.004$ ) were most pronounced (cf. Table 4 and Figure 5). Thus, in addition to the obvious differences in the degree of exploration, quantitative examination of fine root exploitation showed differences between the species. Vangueria infausta had significantly higher mean values for fine root dry weight and 
$R A D$. Strychnos cocculoides had the lowest exploitation and exploration indices among the investigated species. Strychnos spinosa and Grewia flava, with a slightly more intense fine root development than Strychnos cocculoides, are intermediate species.

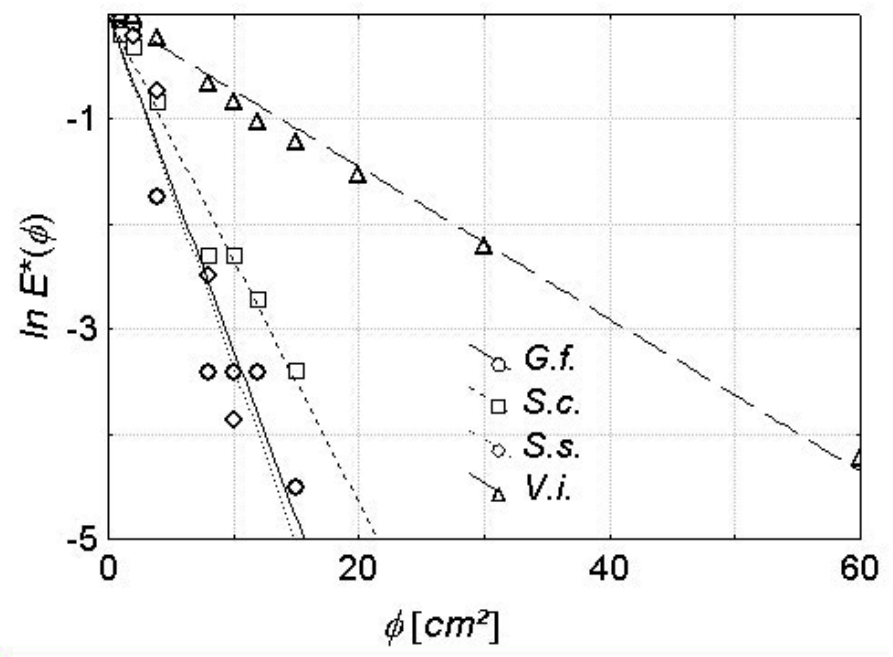

Figure 5. Exploitation index of root area density $(R A D)$ in the logtransformed version. $E^{*}(\phi)$ vs. $\phi$ for each of the investigated species (G.f.: Grewia flava, S.c.: Strychnos cocculoides, S.s.: Strchnos spinosa, V.i.: Vangueria infausta) with displayed regression lines.

It is not surprising that frequently a considerable amount of core samples containing fine roots were also found far beyond the spatial extension of coarse roots (Oppelt et al. 2000). Core samples with fine roots located outside the ISV occurred most commonly in Vangueria infausta (75\%), but also frequently in Grewia flava samples $(54 \%)$, in contrast to both Strychnos species (12-23\%).

Table 4. Exploitation index $E^{*}(\phi)$ for fine root surface area of some arid-zone fruit tree species from Botswana.

\begin{tabular}{ccccc}
\hline \multicolumn{5}{c}{$E^{*}(\phi)$} \\
$\begin{array}{c}(\phi) \\
{\left[\left.\mathrm{cm}^{2}\right|^{-1}\right]}\end{array}$ & $\begin{array}{c}\text { Grewia } \\
\text { flava }\end{array}$ & $\begin{array}{c}\text { Strychnos } \\
\text { cocculoides }\end{array}$ & $\begin{array}{c}\text { Strychnos } \\
\text { spinosa }\end{array}$ & $\begin{array}{c}\text { Vangueria } \\
\text { infausta }\end{array}$ \\
\hline 60.0 & - & - & - & 0.01 \\
30.0 & - & - & - & 0.11 \\
20.0 & - & - & - & 0.21 \\
15.0 & 0.01 & 0.03 & - & 0.29 \\
12.0 & 0.03 & 0.07 & - & 0.36 \\
10.0 & 0.03 & 0.10 & 0.02 & 0.43 \\
8.0 & 0.03 & 0.10 & 0.08 & 0.52 \\
4.0 & 0.18 & 0.43 & 0.48 & 0.80 \\
2.0 & 0.88 & 0.73 & 0.81 & 0.98 \\
1.0 & 0.93 & 0.83 & 0.98 & 1.00 \\
\hline
\end{tabular}




\section{Discussion}

In an investigation of the geometry of the coarse root systems of the four cooccurring species clear differences between the species were shown (Oppelt et al. 2000,2001 ). For both investigations of root ecology and possible agroforestry applications, to fully evaluate the rooting strategies of the considered species, estimation of fine root distribution is essential. This investigation has again revealed clear differences between the species in terms of fine root density. Furthermore, the study shows that systematic core sampling can be a useful tool for quantitative estimation of rooting strategies in terms of exploration and exploitation. With these results obvious species-specific differences in the potential competition with coexisting crops can be detected.

In comparison to other investigations (Hughes et al. 1995, Gandar and Hughes 1988, Ong et al. 1991) very low root length density values (RLD) were found at our sites (cf. Table 2). This may be a result of the plants being native to an extremely arid environment, i.e., as adaptation to or consequence of severe drought conditions. Additionally, it has to be mentioned that most studies use a threshold value for fine roots $<2 \mathrm{~mm}$ (cf. McKenzie et al. 2001), but in our study a diameter of $3 \mathrm{~mm}$ was used to distinguish coarse roots from fine roots. Therefore, care has to be taken in comparing results from other investigations with our (already) low values that would be even more reduced when using a smaller threshold value.

Root dry weight per volume soil is a commonly used parameter to estimate root distribution, as it is easily determined. However, our results clearly show that root dry weight only poorly reflects the differences in fine root morphology found between the species (Figure 1). Both, root length density and root surface area, are important factors for hydraulic properties, but the relationship between $D w t$ and root length was, with the exception of Vangueria infausta, not strong enough to calculate reliable root length densities for the investigated species. Therefore we decided to concentrate on $R A D$ as a parameter representing morphological as well as physiological features. Vangueria infausta clearly had a higher fine root biomass compared to the other species, but this difference was amplified if the surface area of the roots was considered.

The investigation of exploitation index demonstrates the high exploitation of soil by the Vangueria infausta fine root system compared to the other three species. In addition, Vangueria infausta had the highest incidence of fine roots found outside the spatial extension of the coarse roots. These numerical data and the exploration index confirm the impression gained in field investigation that Vangueria infausta forms a root system that intensively explores the soil volume rooted, and can be distinguished from the other species. A comparison between exploration, expressed 
as $E(0)$, and exploitation, quantified as $k$-value, revealed the following results: Vangueria infausta has a low $k$-value, indicating a high exploitative potential. Additionally, this species did also show highest explorative ability. The other species, with approximately 3 -fold higher $k$-values, are representing fine root patterns with obviously lower exploration and also exploitation ability. Although Hughes et al. (1995) used RLD instead of $R A D$, the negative correlation between mean $E(0)$ and mean $k$-values is also in agreement with their results. Therefore the question arises whether a high exploration ability, which can be easily determined by core sampling, indicates already rooting patterns with high potential exploitation and vice versa. Additionally, our results from coarse root patterns revealed a similar ranking between the species concerning exploration, but in terms of exploitation, no speciesdependent differences were found (cf. Part II).

The obvious species-related differences in exploration and exploitation by fine roots can be seen as supporting the results of a previous study of branching patterns of coarse roots (Oppelt et al. 2001). A comparison of the coarse root and fine root patterns suggests that species with coarse roots with more "dichotomous" branching patterns may develop a more intensive fine root system in order to explore the soil volume more thoroughly. In contrast a weak fine root development seems to be correlated with a "herringbone" coarse root structure, occupying a quite large soil volume with low amounts of fine roots and only low branching intensity.

The results show that both coarse root and fine root patterns can vary greatly even in co-occurring species adapted to extreme drought. Furthermore, extremely contrasting rooting strategies, high vs. low exploration and/or exploitation, seem to appear on sites with similar environmental conditions. Therefore, contrasting rooting patterns might be an effective strategy of coexisting plants: spatial sharing of belowground resources, especially under conditions where water and nutrients are severely limiting factors (Noy-Meir 1973, Smith and Nobel 1986, Shmida and Burgess 1988). In addition, this hypothesis has been supported by comparing the rooting habits and water relations of species co-occuring in the same environment, e.g. Cannon (1911), Walter and Stadelmann (1974), Smith et al. (1997).

Colonisation with mycorrhizas facilitates both nutrient, especially $\mathrm{P}$, and water uptake by plants (Ruis-Lozano and Azcon 1995). With the exception of Grewia flava all other species investigated have shown arbuscular mycorrhizal associations, however only Vangueria infausta was highly mycorrhizal (cf. Bohrer et al. 2001), primarily dependent on environmental conditions. Adequate $P$ nutrition is known to greatly increase drought tolerance of plants (Graham and Sylvertson 1984).

Some of the results from Schwinning and Ehleringer (2001) about pulse-driven water uptake are in contrast to our observations concerning the vertical root distribution. 
Fine root distribution, especially in Vangueria infausta, have shown comparatively high root densities in the top soil layers, so we can not confirm the prediction by Schwinning and Ehleringer (2001) that much less shallow root biomass is needed to extract shallow soil water in sandy soils.

Under the aspect of practical applications for agroforestry systems, Vangueria infausta must be considered as the most competitive one between the investigated species in terms of RLD and RAD, whereas Strychnos cocculoides seems to be the less competitive. Strychnos cocculoides has additionally spatial arrangements of fine roots favourable for agroforestry, increasing with depth and low concentration in top soil layers, forming a root safety net (Cadisch et al. 1997). The minor accumulation of fine roots in the upper soil horizons would suggest that in terms of root competition it is highly favourable for growing with annual crops.

This study shows the differences that can occur in the fine roots of co-occurring tree species growing under arid and low nutrient conditions. Clearly, adaptation to arid conditions can occur with highly explorative fine root systems as well as with less explorative systems. Rooting patterns are also influenced by other limiting soil factors, not only aridity. In addition, aboveground adaptations to low water availability have to be taken into account, as within a plant water loss and water acquisition must be in balance. Different aboveground morphologies as found in these species (Oppelt et al. 2001) may require different belowground morphologies. However, the different spatial patterns of fine roots clearly have consequences for possible incorporation in agroforestry systems.

\section{Acknowledgements}

We are grateful for administrative support from Branislav Sloboda and Andrea Polle. Furthermore we thank Mmoloki Botite and Golekwang Phiri for their excellent assistance in fieldwork. Funding was provided by the Commission of the European Union under research grant INCO - DC (ERBIC18CT960035). Technical support from Veld Products Research \& Development was also essential and is gratefully acknowledged.

\section{References}

Berntson G. 1994. Modelling root architecture: are there tradeoffs between efficiency and potential of resource acquisition? New Phytol 127: 483-493.

Bohrer G., Kagan-Zur V., Roth-Bejerano N. and Ward D. 2001. Effects of environmental variables on vesicular-arbuscular mycorrhizal abundance in wild populations of Vangueria infausta. J. Veg. Sci 12(2): 279-288.

Büsgen M. 1905. Studien über die Wurzelsysteme einiger dikotyler Holzpflanzen. Flora 95: 58-94. 
Cadisch G., Rowe E. and van Noordwijk M. 1997. Nutrient harvesting - the treeroot safety net. Agrofor Forum 8: 31-33.

Canon W.A. 1911. The Root Habits of Desert Plants. Carnegie Institution of Washington, Washington D.C., Publication No. 131: 1-96.

Daniel J.N., Ong C.K. and Kumar M.S. 1991. Growth and resource utilization of perennial pigeonpea (Cajanus cajan (L) Millsp.) at the tree-crop interface. Agrofor Syst 16: 177-192.

Fitter A.H. 1985. Functional significance of root morphology and root system architecture. In: Fitter A.H., Atkinson D., Read D.J. and Usher M.B. (eds), Ecological Interactions in Soil, Special Publication of the British Ecological Society, No. 4. Blackwell Oxford, pp 87-106.

Fitter A.H. and Stickland T.R. 1991. Architectural analysis of plant root systems 2. Influence of nutrient supply on architecture in contrasting plant species. New Phytol 118: 383-389.

Gandar P.W. and Hughes K.A. 1988. Kiwifruit root systems. 1. Root-length densities. N. Z. J. Exp. Agric. 16: 35-46.

Graham J.H. and Sylvertson J.P. 1984. Influence of vesicular arbuscular mycorrhiza on the hydraulic conductivity of roots of two citrus rootstocks. New Phytol 97: 277-284.

Hughes K.A., Gandar P.W. and de Silva H.N. 1995. Exploration and exploitation of soil by apple, kiwifruit, peach, Asian pear and grape roots. Plant Soil 175: 301-309.

McKenzie N., Ryan P., Fogarty P. and Wood J. 2001. Sampling, measurement and analytical protocols for carbon estimation in soil, litter and coarse woody debris. National Carbon Accounting System Technical Report No. 14, Australian Greenhouse Office, Canberra, $61 \mathrm{p}$.

Nardini A., Salleo S., Tyree M.T. and Vertovec M. 2000. Influence of the ectomycorrhizas formed by Tuber melanosporum VITT. on hydraulic conductance and water relations of Quercus ilex L. seedlings. Ann For Sci 57: 305-312.

Noy-Meir I. 1973. Desert ecosystems: environment and producers. Ann Rev Ecol Systematics 4: 25-51.

Ong C.K., Corlett J.E., Singh R.P. and Black C.R. 1991. Above and below ground interactions in agroforestry systems. For Ecol Man 45: 45-57.

Oppelt, A.L. Kurth W., Dzierzon H., Jentschke G. and Godbold D.L. 2000. Structure and fractal dimensions of root systems of four co-occurring fruit tree species from Botswana. Ann For Sci 57: 463-475. 
Oppelt A.L., Kurth W. and Godbold D.L. 2001. Topology, scaling relations and Leonardo's rule in root systems from African tree species. Tree Physiol 21: 117-128.

Schwinning S. and Ehleringer J.R. 2001. Water use trade-offs and optimal adaptations to pulse-driven arid ecosystems. J Ecol 89: 464-480.

Shmida A. and Burgess T.L. 1988. Plant growth form strategies and vegetation types in arid environments. Plant Form and Vegetation Structure (eds M.J.A. Werger, P.J.M. v.d. Aart, H.J. During and J.T.A. Verhoeven), pp. 211-241. SPB Academic Publishing. The Hague.

Smith S.D. and Nobel P.S. 1986. Deserts. Photosynthesis in Contrasting Environments (eds N.R. Baker and S.P. Long), pp. 13-62. Elsevier, Amsterdam.

Smith S.D., Monson R.K. and Anderson J.E. 1997. Physiological Ecology of North American Desert Plants. Springer-Verlag, Berlin.

Soil Survey Staff. 1999. Soil Taxonomy - A Basic System of Soil Classification for Making and Interpreting Soil Surveys, 2nd. Edition. USDA (United States Department of Agriculture), NRCS (Natural Resources Conservation Service) Agriculture Handbook No. 436. Washington D.C., 871p.

van Noordwijk M., Lawson G., Soumaré A., Groot J.J.R. and Hairiah K. 1996. Root Distribution of Trees and Crops: Competition and/or Complementarity. In: Ong C.K. and Huxley P. (eds), Tree-Crop Interactions - A Physiological Approach, CAB International in association with ICRAF. Nairobi Kenya, pp 319-364.

Walter H. and Stadelmann E. 1974. A new approach to the water relations of desert plants. Desert Biology Volume 2 (ed. R. Brown). pp. 213-310. Academic Press, New York. 
The following article

Contrasting rooting patterns of some arid-zone fruit tree species from Botswana - II. Coarse root distribution

contributed by:
A.L Oppelt,
W. Kurth,
D.L. Godbold

will be published in:

Agroforestry Systems (2004) 


\title{
Contrasting rooting patterns of some arid-zone fruit tree species from Botswana - II. Coarse root distribution
}

\begin{abstract}
Spatial coarse root distribution of the in situ grown species Strychnos cocculoides BAK., Strychnos spinosa LAM. (Loganiaceae), Vangueria infausta BURCH. (Rubiaceae) and Grewia flava DC. (Tiliaceae) was investigated. The woody roots provide the scaffolding for fine roots, and thus underpin potential competition with fine roots of other species. We developed a method for quantitative description of spatial patterns of coarse roots and correlated fine root distribution with the spatial arrangement of the coarse root systems. In order to estimate different exploration and exploitation strategies, we used the spatial distribution of structural roots within the "individual soil volumes" (ISV) of each root system, and compared the results with other published parameters. We defined a new parameter, "generalized efficiency of exploitation" $G E E(\phi)$, unifying different notions from the literature. Among all the investigated species and dependent on the considered parameter, either Vangueria infausta or Grewia flava had the highest mean values of exploration, hence they could clearly be separated from both Strychnos species. For the exploitation indices, no significant differences were found. However, the generalized efficiency of exploitation $\operatorname{GEE}(\phi)$ was again different between the species. For correlations between coarse and fine roots, the restriction to the ISV was useful to increase the strength of correlations.
\end{abstract}

\section{Introduction}

Although in the field of root research the terms "exploration" and "exploitation" are frequently used expressions, no generally accepted definitions can be found for these terms. Due to the lack of a unique definition, they are applied as more or less intuitive terms in most of the literature. For example, van Noordwijk and co-workers used a very simple definition: they determined the length of the longest (or deepest) root as a rough estimation for "exploration", whereas the "exploitation" is expressed as total length or surface area of live roots (van Noordwijk et al. 1996). In contrast, Berntson (1994), Fitter et al. (1991) and Fitter (1987, 1991a) have much more detailed notions in mind when they calculate "exploitation efficiency" and "exploitation potential". Both Fitter and Berntson concentrated either on cost-benefit relationships of root growth (Fitter 1991b, Fitter et al. 1991) or on exploration and transport characteristics of root systems in connection with topological and geometrical properties (Fitter 1985, 1987; Berntson 1994). However, most of the investigations were conducted at small herbaceous plants. As structural roots determine the 
distribution of fine roots, the architecture of the coarse roots must also be considered to estimate the suitability of certain woody species for agroforestry systems (cf. Fitter 1985). Hence, in addition to an examination of the potential root competition expressed as exploration and exploitation indices of fine roots (cf. Part I), we also investigated and quantified the spatial distribution of structural roots. In analogy to our quantification of exploration and exploitation by fine roots and extending the definitions introduced by Hughes et al. (1995), we apply these definitions to coarse roots in order to quantify competition as well as complementarity aspects.

It is well-known that root interactions are an important issue in agroforestry research since root competition is one of the critical factors for the success or failure of simultaneous agroforestry systems (Schaller et al. 2003). But contrary to most root research, focusing either on coarse or fine roots, the present study analyses both sorts of roots at the same individuals. Additionally, we are also aiming at basic correlations between coarse and fine roots. Our main intention in this investigation was to estimate and compare the potential belowground competition of selected species, which might be useful for commercial and ecological purposes and thus valuable to be integrated into a sustainable concept (cf. Ong et al. 1991, van Noordwijk et al. 1996).

\section{Material and Methods}

\section{Site description}

The investigation site was located in Central District of Botswana between longitude $26^{\circ} 36.26^{\prime}-36.70^{\prime}$ and latitude $22^{\circ} 25.09^{\prime}-25.30^{\prime}$. Sample trees were grown on deep sandy Entisols with no diagnostic horizons (sandveld). According to Soil Survey Staff (1999) this type of soil belongs to the subgroup of "Typic Torripsamments" (LCBG) with rapid infiltration but low water holding capacity. Therefore precipitation moistens the soil to a greater depth than other soils with an aridic moisture regime.

\section{Coarse root sampling}

A detailed description of the investigation site and of the five excavated individuals of each species can be found in Part I and Oppelt et al. (2000, 2001). As minimum threshold for coarse roots a diameter of $3 \mathrm{~mm}$ was chosen, because a reconstruction of the structural roots below this value would not have been possible. Each coarse root system was excavated by manual digging. Starting at the root collar and moving in a distal direction changes in vertical and in horizontal direction of each single root segment was measured, until the threshold value of $3 \mathrm{~mm}$ was reached. After these measurements, roots were removed at their ramification base to measure the 
diameters of each segment. For a more detailed description of data processing see Kurth $(1994,1998)$ and Oppelt et al. $(2000,2001)$.

\section{Spatial analysis of coarse roots}

In order to analyse and quantify the 3-D data of the structural roots, we used the software GRODISC (Dzierzon 2003, Dzierzon and Kurth 2002) to obtain information about spatial coarse root patterns. Values of root length $(R L D)$ and root volume $(R V D)$ densities are obtained by superimposing an imaginary mesh of cubic cells over the three dimensional extension of the whole root system. Based on this calculation we obtained information about the number $n(0)$ of cells which contain parts of the root system. Furthermore, cells including coarse roots were classified according to their total root length or root volume, in order to estimate $n(\phi)$ values for soil exploitation.

\section{Exploration and exploitation by coarse roots}

Due to different interpretations of the expressions "exploration" and "exploitation" we attempted to develop a reasonable and comprehensible definition for these terms. For this purpose we tested the approach used by Hughes et al. (1995), namely their definition of exploitation index $E(\phi)$, which is defined as the proportion of soil volume which contains a quantity of roots higher than a given threshold value $\phi$. The range of $\phi$-values for $R L D$ was between 1 and $100 \mathrm{~cm}$ and the threshold for $R V D$ ranged from 0.1 to $200 \mathrm{~cm}^{3}$.

$E(0)$ is the proportion of soil volume which is exploited by any roots (i.e., RLD >0) and is called the exploration index. Hence, these parameters quantify the soil volume potentially depleted by roots. The key idea behind these definitions is that the presence of only a single root in a given portion of soil volume is already sufficient to "explore" that soil portion, whereas for "exploitation" a minimum amount $\phi$ of root length (or volume) inside the soil portion is required. Of course, both indices depend on the choice of the considered unit of soil volume (grid cell). In addition we considered the parameters called "exploitation efficiency" by Fitter (1987, further developed by Fitter et al. 1991) and "exploitation potential", tested on actual, but far smaller root systems by Berntson (1994). "Exploitation potential" was defined as the sum volume of the depletion zone around each single root (cf. Berntson 1994). On the basis of the distinction made by Hughes et al. (1995) between "exploitation" and "exploration", this root-surrounding volume is, however, a parameter quantifying exploration rather than exploitation. We approximated this volume by the number $n(0)$ of grid cells containing any root and call the approximation "number of explored cells" (NEC). The "exploitation efficiency" was defined by Fitter (1987) and Fitter et al. (1991) as the ratio of total root-system depletion zone volume to total root system 
volume. Adopting the viewpoint of Hughes et al. (1995) again, this parameter must also be interpreted to quantify the efficiency of exploration rather than exploitation. Therefore, we called this parameter "exploration efficiency" $(E E)$. In analogy to $E(\phi)$, we define the generalized efficiency of exploitation $\operatorname{GEE}(\phi)$ as the ratio between the amount $n(\phi)$ of grid cells containing roots with RVD $>\phi$ and total root volume, where $\phi$ is a given threshold value. $E E$ turns out to be the $\operatorname{GEE}(\phi)$ for the special case $\phi=0$. Table 1 gives an overview of our parameters. Grid lengths of $10 \mathrm{~cm}$ for the determination of $N E C, E E$ and $G E E(\phi)$ was used, thus each cube contained one liter of soil.

Table 1. Parameters used to quantify exploration and exploitation of soil and relations to definitions from literature

\begin{tabular}{lcl}
\hline Expression & Formula & Source \\
\hline exploration index $-E(0)$ & $\frac{n(0)}{N}$ & Hughes et al. (1995) \\
exploitation index $-E(\phi)$ & $\frac{n(\phi)}{N}$ & Hughes et al. (1995) \\
number of explored cells - & $n(0)$ & $\begin{array}{l}\text { Berntson (1994): "exploitation } \\
\text { potential" }\end{array}$ \\
exploration efficiency $-E E$ & $\frac{n(0)}{V_{\text {root }}}$ & "exploitation efficiency" \\
generalized efficiency of & $\frac{n(\phi)}{V_{\text {root }}}$ & this article \\
exploitation - GEE $(\phi)$ & $r_{\text {max }}$ or & van Noordwijk (1996): \\
radius or depth of root system & $Z_{\text {max }}$ & "exploration" \\
total root length or surface & $\Sigma R L_{\text {root }}$ & van Noordwijk (1996): \\
area & or & "exploitation" \\
\hline
\end{tabular}

$E(0)$ - approximated proportion of soil volume containing roots (i.e., $R L D>0$ )

$N$ - total number of grid cells

$n(0)$ - number of grid cells containing roots

$E(\phi)$ - approximated proportion of soil volume filled by amount of roots $>$ threshold value $\phi$

$n(\phi)$ - number of grid cells containg roots $>\phi$ (i.e., $R L D>\phi$ )

$V_{\text {root }}$ - sum of root volume of the coarse root system

$r_{\max }$ or $z_{\max }$ - maximum radial extension, resp. depth of coarse root system

$\Sigma R I_{\text {root }}$ or $\Sigma S A R_{\text {root }}$ - sum of root length, resp. total root surface area of coarse roots

\section{Correlations between coarse and fine roots}

As the spatial distribution of the structural roots was known from excavation, we tried to correlate our data from fine root coring with different parameters of the whole coarse root system. The horizontal extension of the coarse roots and the coring depth $(80 \mathrm{~cm})$ were used to calculate an "individual soil volume" (ISV) for each individual (cf. Hughes et al, 1995). Based on this data we determined the number of core samples that are located within the ISV (for a more detailed description see part I). 
All investigated parameters were analysed using one-way ANOVA $(\alpha=0.05)$ and least-significance difference tests, using the species as grouping variable. When necessary, the factor "age" was included as a covariate in order to analyse speciesdependent differences. The software STATISTICA 6.0 was used to carry out the analysis.

\section{Correlations between above and below-ground parameters}

Above ground features, which can be easily determined (e.g., crown projection, crown volume and root collar diameter) were compared with coarse root patterns with means of linear regression models.

\section{Results}

\section{Spatial patterns of coarse roots}

We analysed the coarse root systems for varying sets of rooting depths and horizontal distance. The following strata were distinguished: Top soil layer $(0-20 \mathrm{~cm})$, deeper layers $(0-40 \mathrm{~cm})$ as well as the rooting depth up to $80 \mathrm{~cm}$. Maximum depth of coarse roots was reached by a specimen of Grewia flava $(320 \mathrm{~cm})$ and minimum depth by a Vangueria infausta coarse root system $(112 \mathrm{~cm})$. As individual root systems had a wide range of age and therefore differed considerably in their total coarse root lengths, we calculated the percentage of total root length $(P R L)$ located in different soil layers for each single individual for the sake of easier comparison.

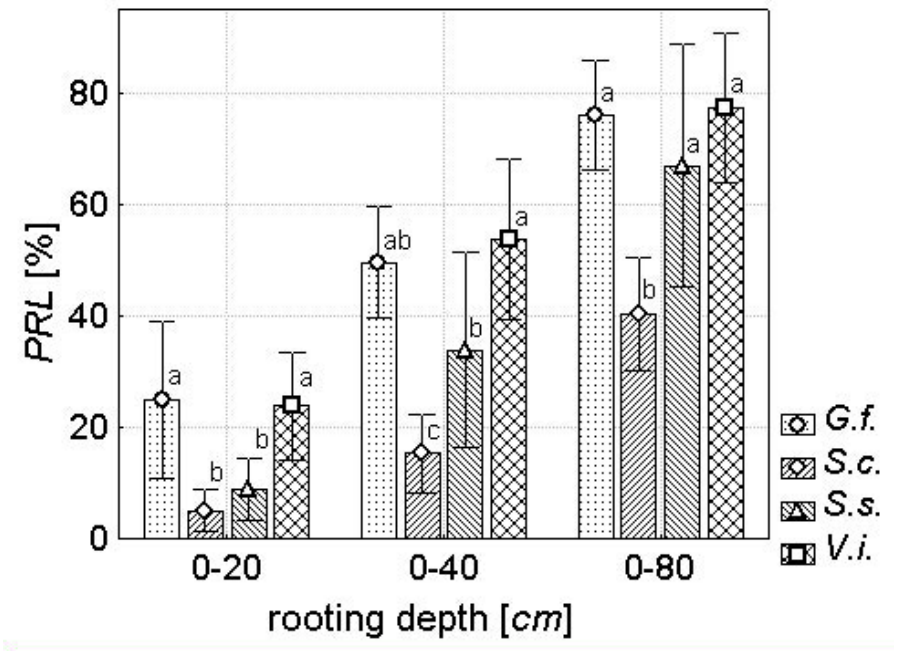

Figure 1. Vertical coarse root distribution expressed as cumulative $P R L$ for some arid-zone fruit tree species from Botswana (G.f: Grewia flava, S.c.: Strychnos cocculoides, S.s.: Strychnos spinosa and V.i.: Vangueria infausta); letters on top of each bar denote whether means are significantly different from one another at $p<0.05$ (Least significance difference test).

The vertical distribution of relative coarse root lengths (cumulative $P R L$ ) for the four species (mean values for each species) is shown in Figure 1 (see also Oppelt et al., 2000). If only the top soil layers $(0-20 \mathrm{~cm})$ are considered, two contrasting groups were obvious, one with high relative root length densities, containing Grewia flava $(25 \%)$ and Vangueria infausta (24\%), the other with distinctively lower values, 
containing both Strychnos species (5-9\%). Although both Strychnos species show a relatively high increase of $P R L$ with increasing soil depth, $(0-40 \mathrm{~cm})$, Strychnos cocculoides (15\%) can still be separated from all the other remaining species. However, Strychnos spinosa (34\%) differs significantly in its $P R L$ values only from Vangueria infausta (54\%). With further increase in depth (i.e., 0-80 cm), Strychnos cocculoides can still be statistically separated from all other investigated species, until a rooting depth of one meter was reached. In terms of vertical coarse root accumulation, Strychnos cocculoides contrast the most with with all the other species, independent of the considered rooting depth (cf. Table 2 and Figure 1).

Table 2. Different $F$ and $p$ values of ANOVA from mean vertical distribution of (cumulative) $P R L$ for coarse root systems of some arid-zone fruit tree species from Botswana for different rooting depths.

\begin{tabular}{ccc}
\hline $\begin{array}{c}\text { rooting } \\
\text { depth [cm] }\end{array}$ & F & $\boldsymbol{p}$ \\
\hline $0-20$ & 6.21 & 0.005 \\
$0-40$ & 9.14 & 0.001 \\
$0-80$ & 6.88 & 0.003 \\
\hline
\end{tabular}

Analysis of horizontal coarse root distribution (cumulative $P R L$ ) was carried out with increasing horizontal distance from the root collar (incremental steps of $100 \mathrm{~cm}$ ), for the different layers of soil depth: 0-20, 0-40 and 0-80 cm (cf. Figure 2).
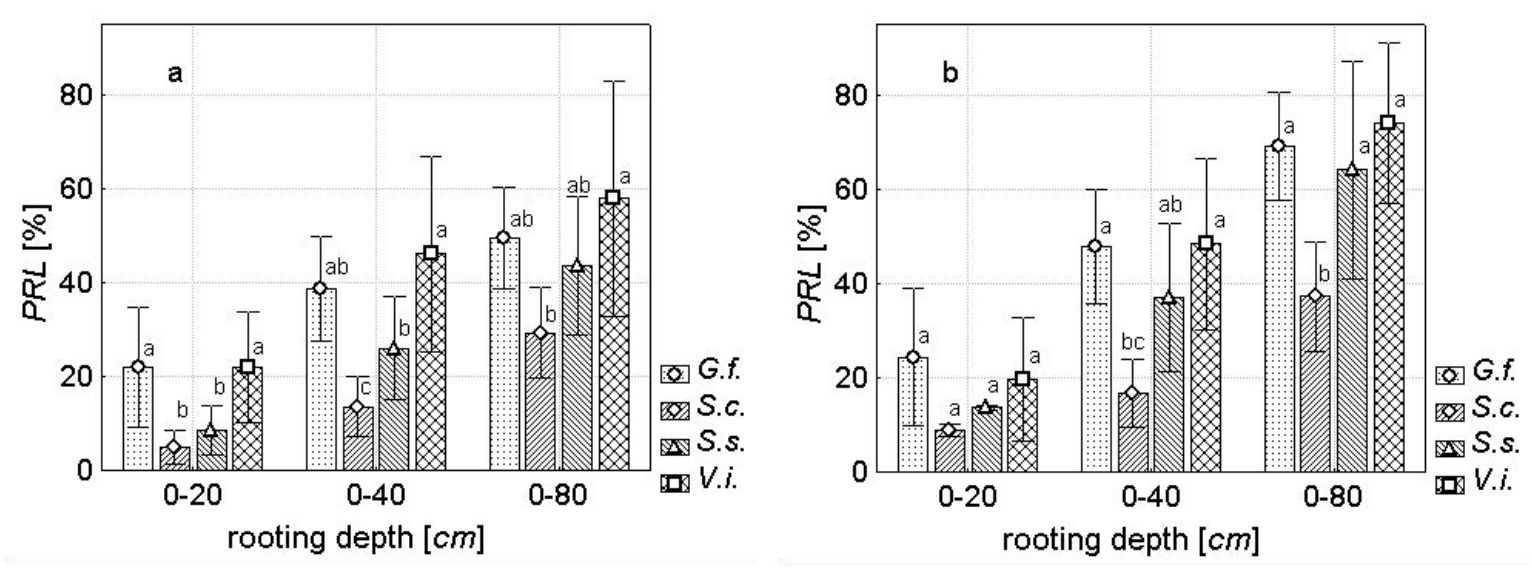

Figure 2. Horizontal coarse root distribution (a: $\leq 100 \mathrm{~cm}$, b: $\leq 200 \mathrm{~cm}$ ) for different sets of rooting depths expressed as cumulative $P R L$ for some arid-zone fruit tree species from Botswana (G.f:: Grewia flava, S.c.: Strychnos cocculoides, S.s.: Strychnos spinosa and V.i.: Vangueria infausta); letters on top of each bar denote whether means are significantly different from one another at $p<0.05$ (Least significance difference test).

A one-way ANOVA with species as factor showed in terms of horizontal and vertical coarse root distribution that the investigated species can be clearly divided into two subgroups: Grewia flava and Vangueria infausta show shallow rooting with relatively high accumulation of coarse roots in the upper soil layers. Increase of $P R L$ of these species tailed-off markedly below a soil depth of approximately $40 \mathrm{~cm}$. In contrast, 
both Strychnos species, especially S. cocculoides, show a markedly different pattern of $P R L$ (cf. Table 3 and Figure 2).

Table 3. Different $F$ and $p$ values of ANOVA from mean horizontal distribution for varying rooting depths (expressed as cumulative $P R L$ ) for coarse root systems of some arid-zone fruit tree species from Botswana.

\begin{tabular}{|c|c|c|c|c|c|c|c|}
\hline \multirow{2}{*}{\multicolumn{2}{|c|}{$\begin{array}{l}\text { horizontal } \\
\text { distance }[\mathrm{cm}]\end{array}$}} & \multicolumn{2}{|c|}{$0-100$} & \multicolumn{2}{|c|}{$0-200$} & \multicolumn{2}{|c|}{$0-300$} \\
\hline & & $\mathrm{F}$ & $p$ & $\mathrm{~F}$ & $p$ & $\mathrm{~F}$ & $p$ \\
\hline \multirow{3}{*}{ 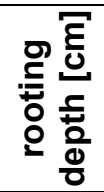 } & $0-20$ & 4.54 & 0.017 & 0.62 & 0.620 & 0.07 & 0.832 \\
\hline & $0-40$ & 5.71 & 0.007 & 4.76 & 0.019 & 1.79 & 0.27 \\
\hline & $0-80$ & 2.72 & 0.079 & 5.31 & 0.011 & 4.46 & 0.035 \\
\hline
\end{tabular}

\section{Exploration by coarse roots}

In terms of different exploration strategies, a one-way ANOVA with species as factor revealed significant differences of the exploration index $E(0)$ for all considered rooting depths (i.e., 0-20, 0-40 and 0-80cm). In contrast to both Strychnos species, with constantly increasing $E(0)$ values for increasing rooting depth, the other species showed decreasing exploration values for a soil depth below $40 \mathrm{~cm}$ (cf. Table 4 and Figure 3). Here, too, S. cocculoides differed the most to the other species.

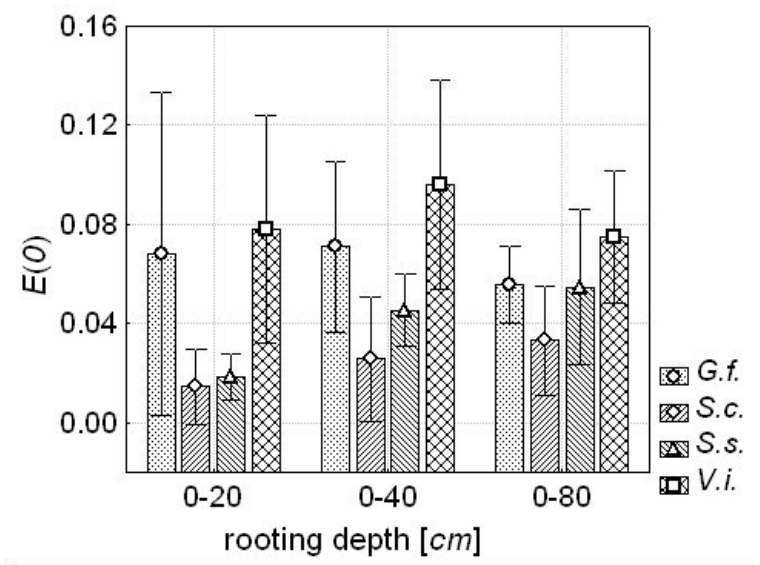

Figure 3. Mean values for vertical distribution of the exploration index $E(0)$ for different rooting depths of some arid-zone fruit tree species from Botswana (G.f.: Grewia flava, S.c.: Strychnos cocculoides, S.s.: S. spinosa and V.i.: Vangueria infausta).

\section{Exploration efficiency and number of explored cells}

A comparison between exploration efficiency $(E E)$ and the number of cells explored $(N E C)$ revealed a positive correlation $\left(r^{2}=0.78\right)$ with minimum values for $S$. cocculoides and maximum values for $G$. flava. The analysis of EE (cf. Fitter 1987, Fitter et al. 1991, Berntson 1994), calculating the volume of explored soil per unit volume of root, revealed clear species-dependent differences. Grewia flava exhibits the highest exploration efficiency, followed by Vangueria infausta, Strychnos spinosa and Strychnos cocculoides. (total root systems: ANOVA $\alpha=0.05, F=12.9, p=$ $1.56 \times 10^{-4} ;$ ISV: ANOVA $\left.\alpha=0.05, F=12.6, p=1.76 \times 10^{-4}\right)$. In terms of NEC, which 
approximates the volume of soil exploited from the number of cubes occupied by any roots, no obvious species-dependent differences can be found (ISV: ANOVA $\alpha=$ $0.05, F=0.92, p=0.45$, total root systems: ANOVA $\alpha=0.05, F=0.44, p=0.73)$. But when we include the age of the root system as a covariate to $N E C$, results improved drastically for the influence of species as a factor for considerations of the restricted soil volume $\left(F=11.92, p=3.8 \times 10^{-4}\right)$, cf. Figure 4 .

Table 4. Mean values of exploration index $E(0)$ for coarse root systems of some arid-zone fruit tree species from Botswana; letters adjacent to each mean denote whether means are significantly different from one another at $p<0.05$ (Least significance difference test).

\begin{tabular}{|c|c|c|c|c|c|c|}
\hline depth [cm] & $0-20$ & & $0-40$ & & $0-80$ & \\
\hline $\begin{array}{l}\text { Grewia } \\
\text { flava }\end{array}$ & 0.068 & $a b$ & 0.071 & $a b$ & 0.056 & $a b$ \\
\hline $\begin{array}{l}\text { Strychnos } \\
\text { cocculoides }\end{array}$ & 0.015 & $b$ & 0.026 & c & 0.033 & b \\
\hline $\begin{array}{l}\text { Strychnos } \\
\text { spinosa }\end{array}$ & 0.019 & b & 0.045 & bc & 0.055 & $a b$ \\
\hline $\begin{array}{l}\text { Vangueria } \\
\text { infausta }\end{array}$ & 0.078 & a & 0.096 & $a$ & 0.075 & a \\
\hline
\end{tabular}

Intuitively, and in agreement with the investigation of Berntson (1994), the correlation between NEC and total coarse root length is strong $\left(r^{2}=0.997\right)$ independent of the considered species. Also a good, but slightly less tight fit was achieved between coarse root volume $\left(r^{2}=0.90\right)$ for the total root system and NEC, thus confirming Berntson's results (cf. Berntson, 1994). But a separation between the species did further enhance the correlation results. In contrast, no size dependent parameters were found to correlate well with exploration efficiency EE. Independent of species and rooting strategy, a certain root length, and to a lower degree also root volume, occupies a similar amount of space, expressed as $n(0)$, which can also be used as an equivalent to the volume of soil explored by structural roots (NEC).

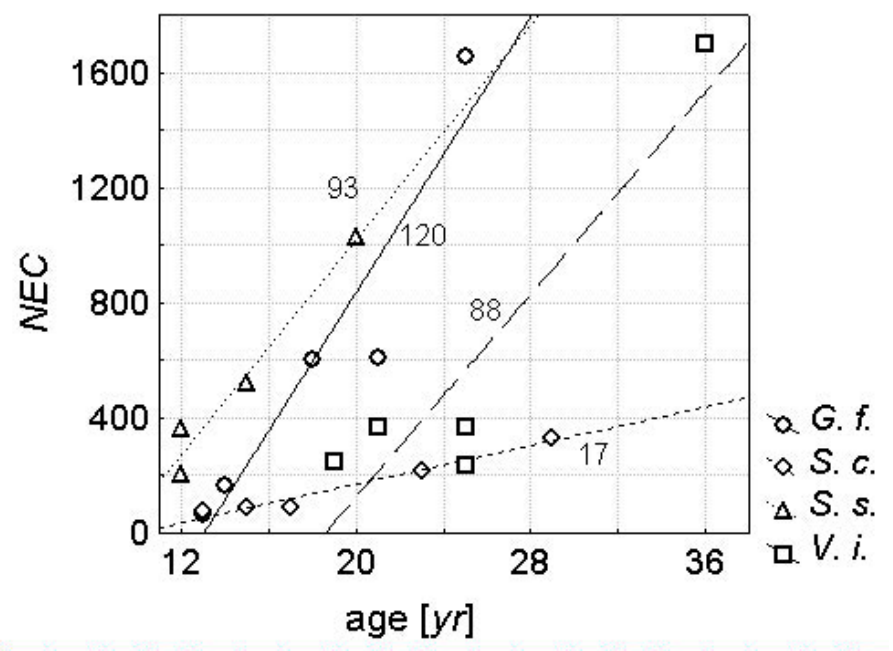

Figure 4. Relationship between age (independent) vs. number of explored cells (NEC) for some arid-zone fruit tree species from Botswana (G.f.: Grewia flava, S.c.: Strychnos cocculoides, S.s.: Strychnos spinosa and V.i.: Vangueria infausta) with regression lines and values of their slopes. 


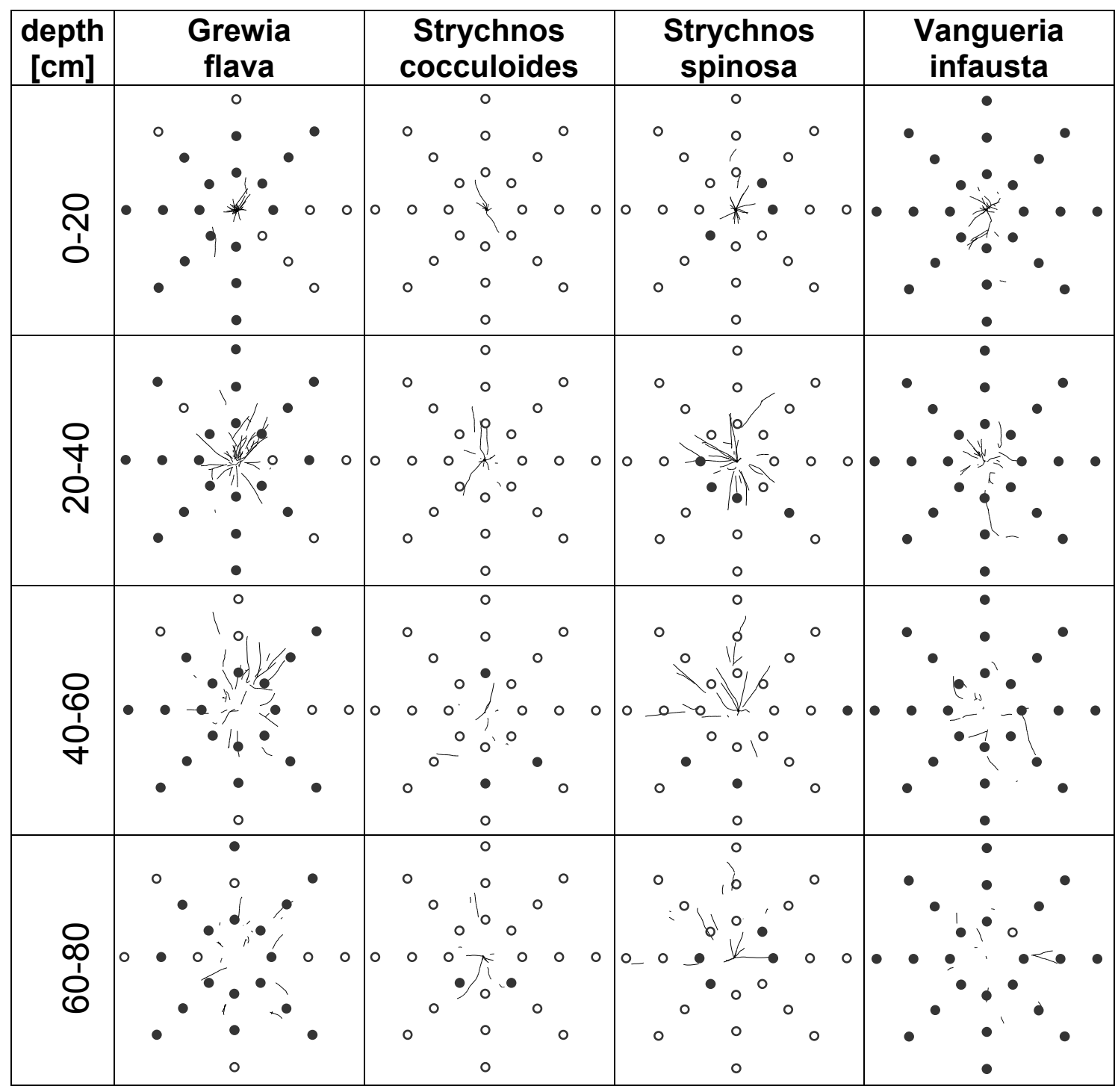

Figure 5. Spatial relation between coarse root patterns and location of cores, either containing $(\bullet)$ or without (o) fine roots for different layers of rooting depths from some aridzone fruit tree species from Botswana.

\section{Exploitation by coarse roots}

In order to quantify $E(\phi)$ we used the parameter "root volume". Differences between $k$ values (exponents relating $\phi$ and $E(\phi)$, see Part I) for the single species were quite low. Hence, in contrast to the investigations of fine roots, species could not be statistically separated from each other. But comparisons between $k$ values must be done carefully, because the used underlying parameters are different ( $S A R$ for fine roots and RLD for coarse roots). Most pronounced differences of the exploitation index between the species occurred at smallest threshold values, but were not significant at the $5 \%$ level. For these small values of $\phi, E(\phi)$ is obviously hardly distinguishable from $E(0)$ and does not give new information. On the other hand, when we consider larger values of $\phi$, the differences between the exploitation indices become even smaller (cf. Table 6). However, clear separations between the species 
were found for the generalized efficiency of exploitation $G E E(\phi)$ defined as the ratio between $n(\phi)$ and sum of root volume $\left(V_{\text {root }}\right)$. Differences between the species were most pronounced for $\phi=2 \mathrm{~cm}^{3}\left(p=2.2 \times 10^{-6}\right)$ and $\phi=2.5 \mathrm{~cm}^{3}\left(p=2.3 \times 10^{-6}\right)$ and least distinct for higher as well as lower threshold values. Considering the whole spectrum of $\phi$ values, between 0.05 and $20 \mathrm{~cm}^{3}$, significant differences between the species were found for all cases where $\phi \leq 10 \mathrm{~cm}^{3}$. The most contrasting species were Grewia flava, with always highest, and Strychnos cocculoides with lowest mean values for $\operatorname{GEE}(\phi)$.

The determination of the rough estimators for "exploration" and "exploitation" used by van Noordwijk (1996) yielded significant results only for the parameter "total root length". In this case a rough indicator for exploration is assumed to be given by the longest (we used the maximum radial extension) or deepest root. In both cases, even with "age" as a covariate, no significant influence of the species could be extracted. Likewise, for total length and total surface area of the coarse root systems, size dependent covariates have to be included. Here, results did strongly improve only for total root length when age was included as a covariate $(F=4.07, p=0.03)$, but not for total surface area $(F=3.04, p=0.07)$. Total root length showed clear positive correlations with the parameters NEC and $E(0)$ which we used to quantify the exploration ability. This is not at all surprising given the definition of NEC (i.e., $n(0)$ ), which can be seen as a discrete version of total root length. Hence the total root length, introduced by van Noordwijk (1996) as an estimator of "exploitation", is in fact related to explorative abilities when we maintain the meaning of "exploration" and "exploitation" adopted from Hughes et al. (1995) and does not yield substantial new information compared with our exploration index $E(0)$. The same holds, to a lesser degree, for total coarse root surface area.

Table 5: Spatial distance relation for the nearest neighbours between coarse roots and fine root containing cores for some arid-zone fruit tree species from Botswana.

\begin{tabular}{lcccccc}
\hline species & \multicolumn{3}{c}{ ISV } & \multicolumn{4}{c}{$\begin{array}{c}\text { outside } \\
\text { ISV } \\
\min [\mathrm{cm}]\end{array}$} & sd \\
\hline $\begin{array}{l}\text { Grewia } \\
\text { flava }\end{array}$ & 39 & $8-26$ & 4.1 & 52 & $7-64$ & 15.0 \\
$\begin{array}{l}\text { Strychnos } \\
\text { cocculoides }\end{array}$ & 18 & $8-22$ & 3.1 & 12 & $7-51$ & 13.3 \\
$\begin{array}{l}\text { Strychnos } \\
\text { spinosa }\end{array}$ & 23 & $8-16$ & 1.7 & 25 & $7-56$ & 13.7 \\
$\begin{array}{l}\text { Vangueria } \\
\text { infausta }\end{array}$ & 143 & $7-48$ & 7.3 & 132 & $7-76$ & 17.1 \\
\hline
\end{tabular}

\section{Morphological features of coarse root systems}

One of the most obvious morphological differences, which clearly distinguishes the coarse root systems of the investigated species, is the existence of a very 
pronounced tap root in both Strychnos species. As this feature is well-known to be an adaptation to arid conditions of some species, we tried to find quantitative differences between the species and we calculated the relative amount of the tap root volume in relation to the total coarse root volume. Grewia flava and Vangueria infausta have only shown a minor relative contribution of the tap root volume (both $23 \%$ ) compared with both Strychnos species. Because of the predominance of the relative tap root volume of Strychnos cocculoides (66\%) this species can be statistically separated from all the other investigated species (ANOVA, $\alpha=0.05, F=8.8, p=0.001$ ).

In terms of radial extension Grewia flava and Vangueria infausta were always more homogeneous in their radial spread of coarse roots than both Strychnos species. As a consequence many of our root system size dependent parameters revealed quite good patterns for the first mentioned species, while results for both Strychnos species for the same factor(s) did considerably deteriorate.
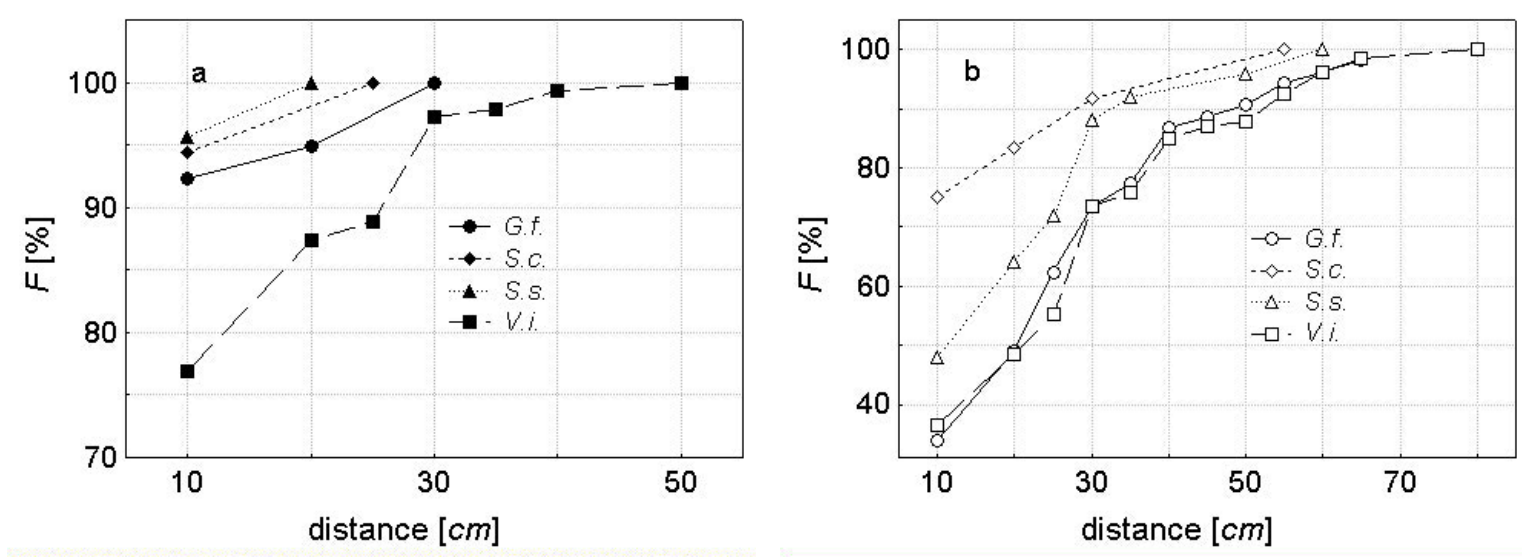

Figure 6. Distance relation between cumulative number of fine root containing cores $(F)$ for some arid-zone fruit tree species from Botswana (G.f: Grewia flava, S.c.: Strychnos cocculoides, S.s.: Strychnos spinosa and V.i.: Vangueria infausta) from (a) inside and (b) outside the ISV.

\section{Correlations between coarse and fine roots}

In Figure 5, one sample of each investigated species is presented to show the spatial relation between coarse root patterns and the location of cores, either void or containing fine roots, for different layers of depth. This example shows that the spatial correspondence between the location of coarse and fine roots must be regarded as a difficult issue. Far beyond the spatial range of coarse roots, cores containing fine roots were frequently found in the case of Vangueria infausta $(75 \%)$, and Grewia flava (54\%, cf. part I). This result might not be surprising, because these species have also shown relatively high exploration ability (for coarse and fine roots as well) and therefore a relatively high amount of cores containing fine roots can be expected also outside the ISV. Both Strychnos species, with distinctively lower 
exploration indices, also revealed lowest relative amount of fine root containing cores (S. cocculoides $12 \%$, S. spinosa $23 \%$ ) from outside the ISV.

Table 6. Mean values for different parameters of exploration and exploitation of some aridzone fruit tree species from Botswana (calculated for the ISV); letters adjacent to each mean denote whether means are significantly different from one another at $p<0.05$ (Least significance difference test).

\begin{tabular}{|c|c|c|c|c|c|c|c|c|c|c|}
\hline species & $\begin{array}{r}\text { NEC } \\
\text { mean }\end{array}$ & & $\begin{array}{r}E(0) \\
\text { mean }\end{array}$ & & $\begin{array}{r}\mathrm{EE} \\
\text { mean }\end{array}$ & & $\begin{array}{c}E(\phi) \\
\phi=2.5 \mathrm{~cm}^{3} \\
\text { mean }\end{array}$ & & $\begin{array}{r}\phi \\
\text { mean }\end{array}$ & $\begin{array}{l}\phi(\phi) \\
\mathrm{cm}^{3}\end{array}$ \\
\hline $\begin{array}{l}\text { Grewia } \\
\text { flava }\end{array}$ & 619 & $a$ & 0.056 & $a$ & 332 & bcd & 0.016 & $a$ & 91 & $\mathrm{bcd}$ \\
\hline $\begin{array}{l}\text { Strychnos } \\
\text { cocculoides }\end{array}$ & 160 & a & 0.033 & $d$ & 80 & ad & 0.012 & a & 27 & acd \\
\hline $\begin{array}{l}\text { Strychnos } \\
\text { spinosa }\end{array}$ & 437 & $a$ & 0.055 & $a$ & 164 & a & 0.025 & $a$ & 57 & $a b$ \\
\hline $\begin{array}{l}\text { Vangueria } \\
\text { infausta }\end{array}$ & 583 & a & 0.075 & $b$ & 202 & $a b$ & 0.023 & $a$ & 64 & $a b$ \\
\hline
\end{tabular}

Nearest neighbour relations, i.e. distance between fine root containing cores and adjacent coarse roots, were analysed in order to assess the local coupling of exploration by coarse and fine roots. Distinctive and common features of the investigated species were found (cf. Table 5, Figure 6). For all the investigated species, and within the ISV, most of the cores containing fine roots (Vangueria infausta: $77 \%$; remaining species: $>90 \%$ ) were located in the immediate vicinity ( $\leq 10$ $\mathrm{cm}$ ) of coarse roots. Outside the spatial range of the coarse roots, $75 \%$ from Strychnos cocculoides fine root samples were located within a distance to coarse roots lower than $10 \mathrm{~cm}$; all other species had distinctly lower values $(35-46 \%)$, indicating that these species spread their fine roots further. Inside the ISV, mean and maximum distances between coarse and fine roots were smaller for both Strychnos species than for Grewia flava and Vangueria infausta. Outside the ISV mean and maximum distances between fine roots and neighbouring coarse roots increased drastically for all the species (cf. Table 5 and Figure 6). Summarizing common trends, distances between nearest neighbours were distinctively lower inside the ISV than outside. And with the exception of $S$. cocculoides all differences were higly significant $(p<0.0001)$ for each species. Hence, for considerations outside the restricted soil volume, distribution of fine roots, expressed as cumulative amount vs. increasing distance, can be well approximated $\left(r^{2}=0.90-0.99\right)$ with logarithmic functions for each single species. But within the ISV, we have not yet found a function that fits well (cf. Figure 6). Outside the ISV, 90 percent of the fine roots were found within 30 respectively $35 \mathrm{~cm}$ for $S$. cocculoides and $S$. spinosa, whereas in the case of Grewia flava $(45 \mathrm{~cm})$ and Vangueria infausta $(55 \mathrm{~cm})$ the same relative amount of fine roots was dispersed within a larger distance. This also explains the differences in the number of root-containing cores outside the ISV. 
When we tested the relationship between total coarse root length and total coarse root volume, respectively, of all individual root systems together and total sampled dry weight of the fine roots, only weak correlations were found for these parameters $\left(r^{2}=0.50\right.$ respectively 0.47$)$. If we further restricted our considerations only to total fine root dry weight within the ISV, correlations did improve considerably $\left(r^{2}=0.76\right.$ respectively 0.84 ) for both total coarse root length and volume. If we omit one specimen from Vangueria infausta (\# 708), sampled during the wet season and probably therefore with an extraordinary high amount of fine root dry weight, our results once again improve drastically $\left(r^{2}=0.85\right.$ and $\left.r^{2}=0.92\right)$ for coarse root length, respectively volume.

\section{Correlations between above-ground and below-ground parameters}

The correlation between root collar diameter, an easily measured parameter, and the fine root dry weight, was tested. As earlier studies (Oppelt et al., 2001) have already revealed good correlations between root collar diameter and total coarse root length respectively root volume, it is not surprising that the correlation between diameter of the root stock and fine root dry weight from the samples located in the ISV also gave good results. Here too, using cores only within the ISV improved the correlation drastically.

Furthermore, the root collar diameter was also well correlated with the explored soil volume $(N E C)$ of the total root systems in the case of Grewia flava $\left(r^{2}=0.95\right)$ and Vangueria infausta $\left(r^{2}=0.93\right)$, but not so well for both Strychnos species $\left(r^{2}=0.42\right.$ for $S$. cocculoides and $r^{2}=0.74$ for $S$. spinosa). This result is also in close accordance with the above mentioned relation between total root length and depleted soil volume and furthermore also with results from earlier studies, investigating the relationship between root collar and total root length (cf. Oppelt et al., 2001).

In contrast to the irregular horizontal projection of Strychnos cocculoides root systems, all other species showed a quite symmetrical spread of coarse roots. Therefore, with the exception of $S$. cocculoides, good correlations were also found between the root collar diameter and the horizontal extension (expressed as mean value between maximum extension in $\mathrm{x}$ and $\mathrm{y}$ direction) for each single coarse root system $\left(r^{2}=0.96,0.46,0.75,0.90\right)$. Furthermore, the root collar diameter can also serve as a good predictor for the maximum radial extension of the coarse root systems of our investigated species $\left(r^{2}=0.98,0.57,0.66,0.86\right)$. 


\section{Discussion}

\section{Methodological aspects}

With increasing limitations on water or nutrients, belowground competition becomes a more critical factor, thus root competition has to be regarded as a very important subject for successful agroforestry systems (Ong et al. 1991). Our investigation revealed that excavation of total coarse root systems combined with additional integration of results from systematic fine root sampling can yield more detailed information and better insights into whole root system architecture (cf. Hughes et al. 1995). This "holistic" aim was achieved through comparison of fine and coarse root patterns. In contrast to Hughes et al. (1995), with a fixed standard volume, we defined an individual soil volume (ISV), based on the horizontal and vertical distribution of coarse roots. With this aid it was possible to find some valuable correlations between both entities and also to determine different distribution patterns of fine roots depending on their location.

\section{Aspects of exploration}

As the distribution of roots of the investigated species have shown to differ considerably (Oppelt et al. 2000, 2001), we focused on different aspects of exploration and exploitation. However, this attempt is not at all an easy issue, because these terms are often used without their meaning being clearly defined. We applied the exploration index $E(0)$, introduced by Hughes et al. (1995) and originally developed for the estimation of fine root distribution, to whole coarse root systems: This attempt turned out to yield a useful parameter to quantify diverse exploration strategies of coarse root systems from co-occuring species. Hence, this information can be used to select promising species under aspects of potential competition. The comparison of the exploration indices for fine and coarse roots gave similar values suggesting that fine root patterns can possibly be used as an indicator of coarse root architecuture. Furthermore, the percentage of soil samples containing fine roots outside the ISV was also well correlated with the indices for $E(0)$ of both, fine and coarse roots. Hence, the index $E(0)$ seems to be suitable to assess the potential competition exerted by fine roots as well as by coarse roots. However, follow-up investigations, including detailed sampling of coarse and fine root distributions of the same individuals, will be necessary to confirm these general conclusions. Based on exploration parameters, Vangueria infausta and Grewia flava appear to be the most competitive species. Hence our observations do not agree with results from Schwinning and Ehleringer (2001) that shallow rooting species (Grewia flava and Vangueria infausta) need a lower root density to extract water from upper soil layers. The simple estimators for exploration or exploitation suggested by van Noordwijk et al. (1996) did not give much reliable information in our case. 


\section{Aspects of exploitation}

For the considered species no correspondence between $k$ values of the exploitation index $E(\phi)$ for fine roots and coarse roots was found. Furthermore, the exploitation index revealed no clear species-specific trends, no matter what $\phi$ value was chosen. Due to this, we tried to find another parameter related to exploitation. The parameter $\operatorname{GEE}(\phi)$ - similar to $E E$ - also includes efficiency considerations and is therefore a measure for exploitation efficiency, with "exploitation" strictly understood in the sense of Hughes et al. (1995). Using GEE $(\phi)$ values, the species could be clearly separated from each other, and also yielded more consistent results for several $\phi$ values. Therefore, for the description of coarse root systems we favour this parameter to describe exploitative differences between the species. In contrast to the investigation on fine roots, the link between exploration and exploitation of coarse roots was not so clear.

\section{Considerations of efficiency}

$E(0)$ and $E(\phi)$ are simple quantitative indices of spatial extension, whereas a consideration of efficiency is inherent in the calculation of $E E$ and $G E E(\phi)$. In contrast to $E(0)$, where $V$. infausta was most competitive, G. flava turned out to be the superior species in terms of $N E C, E E$, and $G E E(\phi)$, followed by Vangueria infausta as the next most competitive species. These species contrast strongly to $S$. cocculoides. In contrast to Berntson (1994), a clear tradeoff between NEC and EE was not shown for the investigated species. Hence, highly explorative species seem to achieve this status also in a more efficient way compared with less explorative species. Comparison between $\operatorname{GEE}(\phi)$ and $E E$ indicates that efficiency of exploitation and efficiency of exploration are closely related to each other, suggesting that strongly exploring species do also exploit the soil intensively ("The winner takes all").

\section{Ecological consequences}

Our study clearly shows that contrasting rooting strategies can occur in the same habitat. These observations are in accordance with well-known patterns from other arid zone plants (Cannon 1911, Weaver 1919) where shallow rooting and highly branched species and, on the other hand, deeper rooting species with herringbonelike root systems were frequently observed. These different structures imply clear consequences for the potential competition: shallow rooting and highly branched systems (Grewia flava and Vangueria infausta) will probably compete more intensively with intermixed crops than the deeper rooting Strychnos species (cf. Fitter 1987). Hence, no generalisation can be drawn concerning "typical" rooting patterns in arid regions. 
Species-dependent differences of the vicinity of fine to coarse roots were found, and can be interpreted as different searching and foraging strategies. Also distinctions (cf. Figure 6) between inside and outside the ISV support the idea of the "tragedy of the commons": a plant should first proliferate roots in unoccupied soil, then in soil occupied by a conspecific competitor, and lastly in soil already occupied by its own roots (Gersani et al. 2001).

Various investigations (Fitter 1987, 1991b) have emphasized the dependency between root morphology and availability of water and nutrients. However, according to our results, the assumption that a more herringbone-like pattern is favoured where soil-derived resources limit plant growth, especially in nutrient-poor and arid environments, is too simple. The existence of contrasting rooting patterns of cooccurring species suggests spatial and temporal sharing of soil-derived resources. Partitioning of the environment has been shown by comparing rooting habits and water relations of other co-existing species in the same environment (e.g. Walter and Stadelmann 1974, Cohen 1970, Cody 1986). Our results agree with these ideas.

\section{Conclusions}

- Coarse root sampling with digital 3D reconstruction of root systems can give insight in different growth and foraging strategies of root systems.

- Parameters quantifying "exploration" and "exploitation" can be defined in a meaningful way, together with corresponding measures of efficiency, based on the approach of Hughes et al. (1995) - and these definitions make also sense for coarse-root systems.

- Co-existing species develop contrasting rooting architectures, which may be an adaptation to reduce competition for limited resources.

- Development of a weak fine root structure is related with a weak coarse root structure and vice versa.

\section{Acknowledgements}

We are grateful for administrative support from Branislav Sloboda and Andrea Polle. Furthermore we thank Mmoloki Botite and Golekwang Phiri for their excellent assistance in fieldwork and Helge Dzierzon for software adaptation. The input of Georg Jentschke in the experimental and sampling design is acknowledged. This work was sponsored by the Commission of the European Union under research grant INCO - DC (ERBIC18CT960035). Technical support from Veld Products Research \& Development was also essential and is gratefully acknowledged. 


\section{References}

Cody M.L. 1986. Structural niches in plant communities. In: Diamond J. and Case T.J. (eds), Community Ecology. Harper \& Row, New York, pp. 381-405.

Cohen D. 1970. The expected efficiency of water utilization in plants under different competition and selection regimes. Israel Journal of Botany 19: 50-54.

Dzierzon H. 2003. Development of methods for characterizing plant and stand architectures and for model comparisons. Dissertation. University of Goettingen.

Dzierzon H. and Kurth W. 2002. LIGNUM: A Finnish tree growth model and its interface to the French AMAPmod database. In: Hölker F. (ed), Scales, Hiearchies and Emergent Properties in Ecological Models. Peter Lang, Frankfurt, pp. 95-104.

Fitter A.H. 1987. An architectural approach to the comparative ecology of plant root systems. New Phytol. 106 (Suppl.): 61-77.

Fitter A.H., Stickland T.R., Harvey M.L. and Wilson G.W. 1991. Architectural analysis of plant root systems 1 . Architectural correlates of exploitation efficiency. New Phytol. 118: 375-382.

Fitter A.H. 1991a. Characteristics and Functions of Root Systems. In: Waisel Y., Eshel A. and Kafkafi U. (eds), Plant roots: the Hidden Half. Marcel Dekker, Inc. New York, pp. 3-25.

Fitter A.H. 1991b. The ecological significance of root System architecture: an economic approach. In: Atkinson D. (ed), Plant Root Growth - An ecological perspective. Blackwell Scientific Publications. Oxford, pp. 229-243.

Gersani M., Brown J.S., O'Brian E.E., Godfrey M.M. and Abramsky Z. 2001. Tradegy of the commons as a result of root competition. J. Ecol. 89: 660-669.

Kurth W. 1994. Growth grammar interpreter GROGRA 2.4. Berichte des Forschungszentrums Waldökosysteme, Vol. B 38. Universität Göttingen. 192 p.

Kurth W. 1998. Die Simulation der Baumarchitektur mit Wachstumsgrammatiken. Wissenschaftlicher Verlag Berlin, Berlin. 327 p.

Schaller M., Schroth G., Beer J. and Jiménez F. 2003. and site characteristics that permit the association of fast-growing trees with crops: the case of Eucalyptus deglupta as coffee shade in Costa Rica. For Ecol Manage 175: 205-215.

Weaver J.E. 1919. The Ecological Relation of Roots. Carnegie Institution of Washington, Publication No. 286. Washington D.C. 



\section{Persönliche Daten}

Name, Vorname

Oppelt, Armin

geboren am 09. Mai 1963 in Bamberg

\section{BERUfLICHE ERFAHRUNGen}

Januar 2001 - Dezember 2001

Softwareentwickler mit ORACLE-Zertifizierung (OCP DBA) ptm-Akademie (München)

Oktober 1996 - Dezember 1999

Promotionsstudium Institut für Forstbotanik (Georg-August-Universität Göttingen)

September 1982 - Juli 1984

Ausbildung als Forstwirt Graf Schenk von Stauffenberg'sche Forstverwaltung)

\section{AusLANDSAUfENTHALTE}

März 1997 - Mai 1999

Botswana: EU-Projekt Feldversuche für Dissertation INCO-DC (ERBIC18CT960035)

November 1995 - März 1996

Tansania: Hospitation bei GTZ Forstwirtschaft in den Tropen und Subtropen

November 1992 - Juli 1993

Costa Rica: Feldstudien für Diplomarbeit

HOCHSCHULSTUDIUM

November 1989 - April 1994

Forstwissenschaften: Ludwig-MaximiliansUniversität München, Dipl.-Forstwirt Univ.

Oktober 1994 - September 1996

Forstwirtschaft in den Tropen und Subtropen: Georg August Universität Göttingen

SChULbILdUNG UND ABSchlÜSSE

\begin{tabular}{ll}
$1984-1986$ & $\begin{array}{l}\text { Berufsoberschule für Agrarwirtschaft: Lands- } \\
\text { hut, Fachgeb. Hochschulreife }\end{array}$ \\
$1980-1982$ & $\begin{array}{l}\text { Fachoberschule: Triesdorf, Fachhochschul- } \\
\text { reife }\end{array}$ \\
$1975-1979$ & Realschule: Eltmann, Mittlere Reife \\
KENNTNISSE & \\
\hline
\end{tabular}

Fremdsprachen

Englisch und Spanisch in Wort und Schrift

Göttingen, 14.01.2004 


